\title{
Does assessment drive students' learning? Clinical context assessment and students' approaches to learning
}

Citation for published version (APA):

Al-Kadri, H. M. F. (2012). Does assessment drive students' learning? Clinical context assessment and students' approaches to learning. [Doctoral Thesis, Maastricht University]. Maastricht University. https://doi.org/10.26481/dis.20120628ha

Document status and date:

Published: 01/01/2012

DOI:

10.26481/dis.20120628ha

Document Version:

Publisher's PDF, also known as Version of record

\section{Please check the document version of this publication:}

- A submitted manuscript is the version of the article upon submission and before peer-review. There can be important differences between the submitted version and the official published version of record.

People interested in the research are advised to contact the author for the final version of the publication, or visit the DOI to the publisher's website.

- The final author version and the galley proof are versions of the publication after peer review.

- The final published version features the final layout of the paper including the volume, issue and page numbers.

Link to publication

\footnotetext{
General rights rights.

- You may freely distribute the URL identifying the publication in the public portal. please follow below link for the End User Agreement:

www.umlib.nl/taverne-license

Take down policy

If you believe that this document breaches copyright please contact us at:

repository@maastrichtuniversity.nl

providing details and we will investigate your claim.
}

Copyright and moral rights for the publications made accessible in the public portal are retained by the authors and/or other copyright owners and it is a condition of accessing publications that users recognise and abide by the legal requirements associated with these

- Users may download and print one copy of any publication from the public portal for the purpose of private study or research.

- You may not further distribute the material or use it for any profit-making activity or commercial gain

If the publication is distributed under the terms of Article $25 \mathrm{fa}$ of the Dutch Copyright Act, indicated by the "Taverne" license above, 


\section{Does Assessment Drive Students' Learning?}

Clinical Context Assessment and Students' Approaches to Learning

Hanan Mohammad Fouad Al-Kadri 
The studies in this dissertation was carried out at

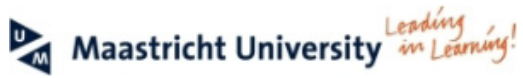

in the School of Health Professions Education

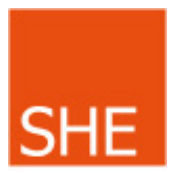

Hanan Mohammad Fouad Al-Kadri

King Saud bin Abdulaziz University for Health Sciences

Riyadh, Saudi Arabia 


\section{Promoter}

Prof. Dr. C. P. M. van der Vleuten

Maastricht University, Maastricht, The Netherlands

\section{Co-promoter}

Associate Prof. Chris Roberts

Sydney Medical School, Sydney, Australia

\section{Assessment Committee}

Prof. Dr. Youssef Al Eissa, MD

Prof. Dr. Richard Koopmans, MD

Prof. Dr. Fedde Scheele, MD

Prof. Dr. Albert Scherpbier, PhD

Prof. Dr. Bas Verhoeven, MD 


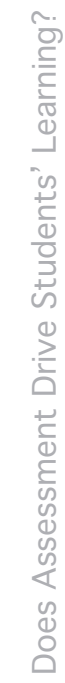




\section{Contents}

$\begin{array}{lll}\text { Chapter Title Page } & \text { Par }\end{array}$

Chapter 1 Introduction

7

Exploring factors affecting undergraduate medical students'study Chapter 2 strategies in the clinical years: a qualitative study. Published under the same title in Adv Health Sci Educ Theory Pract. 16(5):553-67

Students' and teachers' perceptions of clinical assessment Chapter 3 program: A qualitative study in a PBL curriculum. Published under the same title in BMC Res Notes 2, 263.

Students' perceptions of the impact of assessment on approaches

Chapter 4 to learning: a comparison between two medical schools with similar curricula. Published under the same title in Int $\mathbf{J}$ Med 69

Educ. 2, 44-52.

Self-assessment and students' study strategies in a community

Chapter 5 of clinical practice: A aualitative study. Provisionally accepted 93 in Medical Education Online Journal.

Exploring assessment factors contributing to students' study Chapter 6 Strategies; Literature peview. Accepted in Medical Teacher Journal Saudi Supplement.

Chapter 7 General discussion

Chapter $8 \quad$ Summary (English)

Chapter 9 Summary-Samenvatting (Dutch)

Curriculum Vitae

SHE Dissertations Series

185 

Chapter 1

\section{Introduction}



Assessment steers learning is a statement that has been presented by Newble as early as 1983 (Newble, 1988). Since then this statement is viewed as well-proven despite the fact that supportive evidence was quite limited. Several researches have been published aiming to explore the relation between assessment and learning. Until recently, literature was not firmly sure of the effect of assessment and this effect direction on students' learning (Norman et al., 2010).

Looking back into assessment history, adopting the psychometric theories for several decades has focused on factors and indicators that are related to assessment quality (Schuwirth and van der Vleuten, 2004). These indicators such as reliability; where we achieve stable estimates of students' ability through the used assessment and validity; where we refer to the extent to which a test's content is representative of the actual skills learned and whether this test can allow accurate conclusions concerning students' achievement. In another word, when we say the test is reliable, it means that if we repeat similar tests using the same tool, we guarantee achieving similar results for students with similar ability and knowledge levels. And when we say the test is valid, it means that it is testing what should be tested based on the curriculum objectives.

The implementation of the psychometric theory has resulted in several outcomes: 1) medical educators became keen to look for an assessment with measurable outcomes that are presented in numbers of which assessment quality indicators can be calculated, 2) summative assessment was given higher importance in comparison with formative assessment, 3) teachers test selection was directed to achieve high test quality (high validity and reliability) rather than focusing on the test educational impacts. (Schuwirth and van der Vleuten, 2004), 4) teachers have avoided practicing a bi-directional process between them and their students aiming to enhance, recognize and respond to students' learning (Cowie and Bell, 1999), 5) teachers made little effort to implement formative assessment as a method to help the learning process (Reay and Wiliam, 1999) (Black and 


\section{Introduction}

Wiliam, 1998b) and finally and 6) teachers put more stress on the value of summative assessment that summarize the development of learners numerically at a given time particularly while dealing with high stakes assessment.

Assessment then became a method to rank students or decide their pass or fail rather than an instrument to enhance their learning. Its influence was mainly on assessment of students learning rather than assessment for students learning enhancement. This influence was on both teachers particularly their "teaching methods" that was turned to be test oriented (Harlen, 2005) and students particularly their "study practice" that became relying more on rehearsal reiterative practice of what might come in the exam (Harlen, 2005).

The question now is: what is the effect of this shift towards summative assessment rather than formative assessment on students learning?.

Existing research on the effects of summative and formative assessment on students' learning is unequivocal. Whilst some researchers claim that formative assessment is more effective than summative assessment in enhancing students learning (Rushton, 2005), others disagree (Torrance and Pryor, 1998, Hattie, 1978).

Experimental studies have shown that periodic formative assessments can enhance learning over equivalent time spent in study (Roediger and Karpicke, 2006). Moreover, providing students with non-judgmental feedback helps in identifying where they are in relation to learning objectives and helping students to work towards learning goals rather than performance goals. Some researchers have even concluded that feedback and formative assessment produce the most powerful effect on student achievement (Hattie, 1978, Narciss, 2004). Formative assessment appears to play a larger role in increasing student achievement than does a reduction in class size or an increase in teachers' content knowledge (Dylan and Thompson, 2008) and it appears to be the 
key factor contributing to the implementation of assessment for learning where all activities undertaken by teachers and their students help in providing information to be used as feedback and to modify the teaching and learning activities in which they are engaged in (Black and Wiliam, 1998b). On the other hand, summative assessment is a proven way of eliciting evidence of student achievement and a valuable tool that discriminate between students of differing abilities (Black and Dylan, 2009). Overall, it is not clear using which type of assessment; summative that represent the main element of assessment of learning or formative that represent the main element of assessment for learning, and under which circumstances students learning will be positively or negatively influenced.

\section{Assessment of learning and assessment for learning}

In contrast to the psychometric theory, assessment for learning moves the educational focus from an individual instrument into programmatic assessment where we tend to use multiple assessment instruments each of which is directed to cover particular aspect of the intended educational objectives (Schuwirth and Van der Vleuten, 2011, van der Vleuten and Schuwirth, 2005). In this approach we don't necessarily replace the old assessment tools with the new ones, rather we supplement the traditional tools with tools that focus more on the tests educational impact (Prescott et al., 2002). Based on this new concept, assessment for learning was defined by Schuwieth et al as an information-rich approach in which a program of assessment is used to collect and combine information from various sources to inform about the strengths and weaknesses of each individual student, with the purpose to optimize their learning (Schuwirth and Van der Vleuten, 2011). To enhance this information-rich approach, we need to match between the implemented educational program and the assessment method with reinforcement on the educational objectives of the assessment program. Such reinforcement will prevent students from using a 'hidden curriculum (Muijtjens et al., 1998). Through this 


\section{Introduction}

approach, we move from thinking of assessment as a trial to capture the whole picture of students performance with one single best instrument towards perceiving assessment as a program which is completely intertwined with education. We move also toward assessment that consist an integral part of education, where the main question is not pass or fail rather, to determine the strengths and weaknesses of the candidates and to optimize their individual learning pathways (Schuwirth and van der Vleuten, 2004). Overall we can say that the central key of assessment for learning is the assessment set up. This set up should allow obtaining the whole picture of a student's competence by a careful selection of assessment methods, formulation of rules and regulations and design of organizational systems that support the practice of assessment for learning. To achieve this goal, we need a broad and highly informative assessment program that is capable of exposing all aspects of individual students learning and showing all the nuances. Although the notions of programmatic assessment and assessment for learning have shown to be appealing ones, it still require more concrete development through better understanding of the effect of different assessment tools implementations on students' study habits and strategies.

\section{Students' study strategies}

Research into student learning initially built up evidence about the relationships of motivation and study methods with academic performance (Biggs, 1970, Biggs, 1976, Entwistle and Entwistle, 1970, Schmeck et al., 1977). Researchers stressed the importance of the student's own effort and application in determining levels of academic achievement. The responsibility for high achievement was seen as the student's alone, with effort explained in terms of the student's motivation and application represented through study habits (Biggs, 1970, Biggs, 1976, Entwistle and Entwistle, 1970). At that time, students' study strategies were evaluated in isolation of the implemented assessment practiced, teaching methods and other personal and contextual factors that 
may have an influence on student's study strategies. Subsequently, the link between teaching methods and study strategies was demonstrated (Biggs, 1999, Ramsden, 1992). The effect of teaching was found to go well beyond the direct influence of the teacher to include other features of the whole teaching-learning environment, particularly assessment procedures (Biggs, 1999).

The initial learning style inventories have emphasized the relative stability of students' study strategies and were used to predict students' future academic performance (Biggs, 1970, Biggs, 1976, Entwistle and Entwistle, 1970, Schmeck et al., 1977). Based on the early inventories students' study strategies found to reflect three basic learning models (Broadbent, 1966): surface learning, involving "repetition of analyses already carried out" and usually directed at reproducing information; deep level learning, using "a greater degree of semantic or cognitive analysis" usually aimed at gaining insight and understanding and "assessment driven category", named a strategic approach to studying (Entwistle and McCune, 2004). Researches on students' study approaches have progressed to focus more on the effect of teaching-learning environment (Biggs, 1999) and have investigated the differing ways students interpret the requirements of a task within a specific learning context (Marton and Saljo, 1997). Students' study approaches were found to be unstable swinging between superficial, deep and strategic approach as a result of different contextual and personal factors. Moreover, inventories have emphasized students' self-conscious reflection on studying, drawing on the ideas of "meta-cognition" and "self-regulation" (McKeachie, 1990, Vermunt, 1996, Vermunt, 1998). Students' study approaches categorizations were then modified to deep, reflective and elaborative approach and superficial with serial, reiterative, or rehearsal approach. A third approach of studying that describes methodical, well-organized studying linked to effort that is done by the students based on certain motivation and aiming for certain achievement such as passing exam or achieving high scores or leaving good impression and obtaining certain recommendation. This particular strategy is called effort and 
achievement motivation study strategy and has replaced the strategic strategy approach. To what extent teaching learning environment such as clinical context of practice is affecting students' perception of their learning and their study strategies is a major area that needs to be researched.

From the above, it is clear that learning functions play a central role in the theory on regulation of learning processes (Vermunt, 1989). However, little is known about the manner in which students carry out and regulate these functions in a clinical educational context. Insight into these processes can make an important contribution to the improvement of clinical education and its instructional process.

In the clinical years, we expected to find a spectrum of students' responsiveness to stressful situations, in terms of study strategies, such as dealing with patient problems, assessment and clinical supervision. A question with important implications for teaching and learning then arises: under which conditions do students use which study strategies? And furthermore, which factors contribute to students' use of particular strategies? To our knowledge, no study has specifically explored this issue within the clinical learning environment. The increasing need for information on training and certification in higher education and the increasing requirements of universities to justify effectiveness and efficacy of their teaching put more importance to the answers of these questions.

\section{Students' study strategies in the clinical context or "community of clinical practice"}

The term "community of practice" was first described by Lave and Wenger (1990) as learning through practice and participation. It describes the function of a group of people who have a variety of experiences but share an interest or a profession. They share information and experiences, learn from each other and have similar opportunities to develop (Lave and Wenger, 1990). Lave and Wenger believe that 
everybody is involved in a number of communities of practice whether at work, school or at home. The structural characteristics of these communities of practice are defined as a domain of knowledge a notion of community and a practice (Wenger, 1998).

In the clinical context, students' knowledge about their training requirements, their assessment and their supervision create a common ground for students' learning, guide their study strategies and give meaning to their actions and interactions. The presence of a community of students and supervisors in the clinical context create the social fabric of students' learning, relations with the surroundings and their interrelations. It appears that students' learning in the clinical context and their social experiences are a new example of "community of practice" that can be called "community of clinical practice". Students' study strategies in the community of clinical practice their actions and interactions are widely affected by the clinical environment, direct patients contact, work based assessment and faculty given feedback.

Encouragement of appropriate application of clinical knowledge in the clinical context was reported to foster a deep approach to learning and enhance performance on clinical assessments (Norton, 2004). It has been also reported that when people use ineffective strategies to achieve success and satisfaction, they suffer a dual burden. Not only do they reach erroneous conclusions and make unfortunate choices, but their incompetence also robs them of the ability to realize these consequences (Kruger and Dunning, 1999). Therefore, to achieve effective study strategy in a clinical context, students need to be empowered with specific skills through specific steps and protocols. They have initially to be able to assess themselves and identify their knowledge and skills deficiencies. This process of self-assessment consists of two main steps: 1) identifying self-ability in comparison to the required standards for a task and 2) seeking and using constructive feedback. 


\section{Self assessment and students' study strategies in the community of clinical practice}

Self-assessment is widely reported to offer numerous advantages to the learner. The resulting dialogue between students and teachers and the skills acquired through selfassessment processes are found to lead to enhancement of students' achievement, critical awareness, and reflection on learning (Black and Wiliam, 1998a, Dearnley and Meddings, 2007). Therefore, self-assessment is assumed to promotes a deeper approach to learning (Ramsden, 1992). However, research suggests that both students' perception and practice of self assessment are unstable skills that can be affected by different variables such as students' gender, degree of ability, length of practice, broadness of the studied area (Falchikov and Boud, 1989) and the presence of a well developed sense of the criteria which they should use to judge and effectively interpret their own work (Boud, 1989). This instability leads to inconsistencies in the effect of self-assessment practice on students' self regulation and even on their self-direction as lifelong learners (Kirbya and Downsa, 2007). Moreover, it leads to inconsistencies in the strategies used by the students when they practice self-assessment leading to either an over or under estimation of their performance. It also results in poor agreement between students' self-assessment ratings and their supervisors' in course performance, particularly students' rating as measured by the faculty (Woolliscroft et al., 1993).

The process of self assessment is composed of two main steps (Eva and Regehr, 2008): 1) identification of self ability in comparison to the required standards for a task and 2) seeking and directing performance based on constructive feedback. The skill of how to identify self abilities and how to seek feedback and utilize it is a crucial part of self assessment practice.

In the community of clinical practice, self-assessment is interpreted as a learning experience that can be instrumental in stimulating learning by identifying standards 
of excellence and deficits and providing suggestions for improvement (Colliver et al., 2005). Teachers in the clinical setup or in the "community of clinical practice", make an invaluable contribution to students' learning (Dolmans et al., 2002, Stern et al., 2000). This contribution is very important since research and experience have shown that learning in the workplace is implicit, haphazard and dictated by the demands of the workplace (Eraut, 2004). Clinical teachers then face the difficult task of meeting high performance standards of both service and teaching, respond to students self assessment, identify students' weaknesses and strengths and be able to provide constructive feedback that guide students towards high quality study strategy that results in the development of competent individuals. However, the literature lacks information on 1) what type of students' study strategy is linked or practiced in response to students' self assessment and more importantly 2) which component of self assessment is responsible for which type of study strategy in the community of clinical practice.

\section{Overview and research questions}

From what we have introduced above, it appears that there are several vague areas in the literature concerning the relation of the implemented assessment program and students' study strategies. Different effects of assessment on learning have been reported in the literature. Some studies have shown that students' approaches to learning and their retention of knowledge differ across a range of assessment methods, learning environment and assessment demands as perceived by students (Larsen et al., 2008, Reid et al., 2007). Although many researchers emphasize this relationship (Biggs, 1999, Newble and Jaeger, 1983), it continues to be poorly understood particularly with regard to the following aspects: 1) the persistent incongruence between curricular and assessment objectives; 2) the purpose of assessment methods (formative/summative) (Cooksey et al., 2007). Moreover, little is known about the manner in which students carry out and regulate their learning functions in response to their assessment in a 
clinical educational context (Vermunt, 1989). In the clinical years, we expected to find a spectrum of students' responsiveness to stressful situations. A question with important implications for teaching and learning then arises: under which situation do students use which study strategies? And furthermore, which assessment factors contribute to students' use of particular strategies? What are the barriers to a deep learning approach as perceived by students? How and why students differ in their approaches to learning and which barriers stand in the way of good assessment and learning.

This dissertation is presented to assess and discuss the effect of assessment on students approach to learning in the clinical context or "community of clinical practice". We utilized a qualitative approach and literature review in exploring students' and teachers' perception of the implemented assessment in different clinical contexts aiming to answer our research questions.

We used the previous two approaches to generate a rich understanding of the full range of opinions and experiences of students when they are exposed to the implemented assessment and to examine what happens when students direct their study strategies to fit with assessment rather than learning objectives. Our aim was to gain insight into how students experience their assessment program and this experience effect and direction on their study strategies.

Taking our researches aim collectively, we can summarize our research questions as the following (figure 1):

1- What is the relationship between the learning environment, assessment demands as perceived by students, and students' approaches to learning in the community of clinical practice? (chapter 2).

2- What are the different assessment factors including feedback contributing to students' study strategies in the clinical context or "community of clinical 


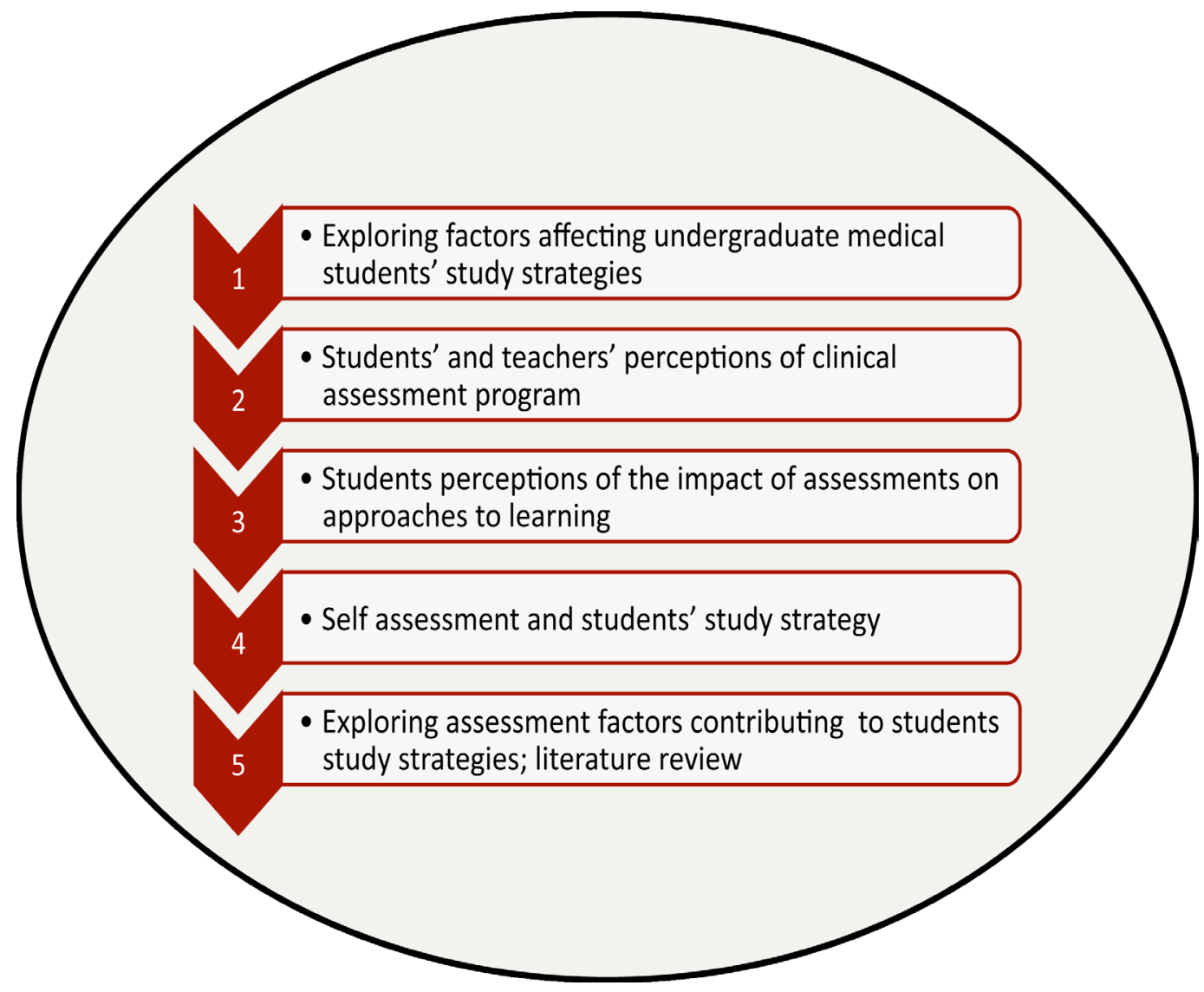

Figure 1: The various researches performed in this dissertation to assess the impact of assessment on students' approaches of learning

practice"? Can these factors be classified as promoting one of the different known study strategies (chapter 3) and (chapter 4) and (chapter 5, under revision revision).

3- Where our results do stand within the available research on assessment effects on students learning in general and within a clinical context specifically? (Chapter 6, under revision). 
In this dissertation the following chapters were addressed to represent the following:

Chapters 2: describes the first research that was considered the baseline of our work. In that research, we explored factors affecting students' study strategies in the clinical context.

Chapter 3: describes our second research. In that work, we have explored students and teachers perceptions of their implemented assessment. We utilized a qualitative approach and we have interviewed both students and their teachers in their clinical practice. We utilized the data obtained of our first research to support our interview questions and deeply explore students' perceptions.

Chapters 4: This chapter deals with our third research in which we have worked on assessing the effect of different contextual and personal factors on students' perception of their assessment and the resultant study strategies. We compared between two different colleges that are implementing similar curriculum but different assessment programs. These two colleges are located in two different geographic locations (Saudi Arabia and Australia) and have very different cultural backgrounds.

Chapter 5: It presents our fourth research in which we have explored the role of self assessment process in driving students learning and directing their study strategies in the clinical context. In this research we exposed the students to different contextual factors such as "knowledge and training on self assessment" and "summative assessment" to deeply explore the role of self assessment with its two main components "self assessment" and "feedback" on students' learning.

Chapter 6: It presents our fifth research in which we worked through conducting a literature review in identifying where our results do stand in between all of the conducted researches on assessment effects on students learning in general and within a clinical context specifically?. 


\section{References}

BIGGS, J. (1999) Teaching for Quality Learning at University, Buckingham, SRHE and Open University Press.

BIGGS, J. B. (1970) Faculty pattern in study behaviour. Aust. J. Psychol, 22, 161-174.

BIGGS, J. B. (1976) Dimensions of study behaviour: Another look at a.t.i. Br. J. Educ. Psychol, 46, 6880.

BLACK, P. \& DYLAN, W. (2009) Developing the theory of formative assessment. Educ Asse Eval Acc, 21, 5-31.

BLACK, P. \& WILIAM, D. (1998a) Assessment and classroom learning. Assessment in Education, 5, 7-74.

BLACK, P. \& WILIAM, D. (1998b) Inside the black box: Raising standards through classroom assessment. Phi Delta Kappan, 80, 139-149.

BOUD, D. (1989) The use of self-assessment in student grading. Assessment and Evaluation in Higher Education, 14, 20-30.

BROADBENT, D. E. (1966) The well-ordered mind. Am. Educ. Res. J, 3 281-295.

COLLIVER, J. A., VERHULST, S. J. \& BARROWS, H. S. (2005) Self-assessment in medical practice: a further concern about the conventional research paradigm. Teach Learn Med, 17, 200-1.

COOKSEY, R. W., FREEBODY, P. \& WYATT-SMITH, C. (2007) Educational Research and Evaluation, $13,401-434$.

COWIE, B. \& BELL, B. (1999) A model of formative assessment in science education. Assessment in Education, 6, 101-116.

DEARNLEY, C. A. \& MEDDINGS, F. S. (2007) Student self-assessment and its impact on learning - a pilot study. Nurse Educ Today, 27, 333-40.

DOLMANS, D. H., WOLFHAGEN, H. A., ESSED, G. G., SCHERPBIER, A. J. \& VAN DER VLEUTEN, C. P. (2002) Students' perceptions of relationships between some educational variables in the out-patient setting. Med Educ, 36, 735-41.

DYLAN, W. \& THOMPSON, M. (2008) Integrating assessment with instruction: What will it take to make it work? Future of assessment: Shaping teaching and learning. New York, Lawrence Erlbaum Associates.

ENTWISTLE, N. \& MCCUNE, V. (2004) The Conceptual Bases of Study Strategy Inventories. Educational Psychology Review, 16, 325-345.

ENTWISTLE, N. J. \& ENTWISTLE, D. M. (1970) The relationships between personality, study methods and academic performance. Br. J. Educ. Psychol, 40 132-141.

ERAUT, M. (2004) Informal learning in the workplace. Studies in Continuing Education, 26, 247-273.

EVA, K. W. \& REGEHR, G. (2008) "I'll never play professional football” and other fallacies of selfassessment. J Contin Educ Health Prof, 28, 14-9.

FALCHIKOV, N. \& BOUD, D. (1989) Student Self-Assessment in Higher Education: A Meta-Analysis. Review of Educational Research, 59, 395-430.

HARLEN, W. (2005) Teachers' summative practices and assessment for learning tensions and synergies. The Curriculum Journal, 16, 207 - 223.

HATTIE, J. A. (1978) Identifying the salient factors of a model of student learning: a synthesis of meta- 


\section{Introduction}

analyses. International Journal of Educational Research, 11, 187-212.

KIRBYA, N. F. \& DOWNSA, C. T. (2007) Self-assessment and the disadvantaged student: potential for encouraging self-regulated learning? Assessment \& Evaluation in Higher Education, 32, 475-494.

KRUGER, J. \& DUNNING, D. (1999) Unskilled and unaware of it: how difficulties in recognizing one's own incompetence lead to inflated self-assessments. J Pers Soc Psychol, 77, 1121-34.

LARSEN, D., BUTLER, A. \& ROEDIGER, H. (2008) Test enhanced learning in medical education. Med Edu, 42, 959-966.

LAVE, J. \& WENGER, E. (1990) Situated Learning: Legitimate Periperal Participation, Cambridge, UK, Cambridge University Press.

MARTON, F. \& SALJO, R. (1997) Approaches to learning. IN MARTON, F., HOUNSELL, D. J. \& ENTWISTLE, N. J. (Eds.) The Experience of Learning. 2nd ed. Edinburgh, UK, Scottish Academic.

MCKEACHIE, W. J. (1990) Research on college teaching: The historical background. J. Educ. Psychol, 82, 189-200.

MUiJtJens, A. M., HOOGENBOOM, R. J., VERWIJNEN, G. M. \& VAN DER VleUTEN, C. P. (1998) Relative or Absolute Standards in Assessing Medical Knowledge Using Progress Tests. Adv Health Sci Educ Theory Pract, 3, 81-87.

NARCISS, S. (2004) The impact of informative tutoring feedback and self-efficacy on motivation and achievement in concept learning. Exp Psychol, 51, 214-28.

NEWBLE, D. \& JAEGER, K. (1983) The effect of assessment and examination on the learning of medical students. Med Educ, 17, 165-171.

NEWBLE, D. I. (1988) Eight years' experience with a structured clinical examination. Med Educ, 22 , 200-4.

NORMAN, G., NEVILLE, A., BLAKE, J. M. \& MUELLER, B. (2010) Assessment steers learning down the right road: impact of progress testing on licensing examination performance. Med Teach, 32, 496-9.

NORTON, L. (2004) Using assessment criteria as learning criteria: a case study in psychology. Assessment and Evaluation in higher education, 29, 687-702.

PRESCOTT, L. E., NORCINI, J. J., MCKINLAY, P. \& RENNIE, J. S. (2002) Facing the challenges of competency-based assessment of postgraduate dental training: Longitudinal Evaluation of Performance (LEP). Med Educ, 36, 92-7.

RAMSDEN, P. (1992) Learning to teach in higher education, London, Routeledge

REAY, D. \& WILIAM, D. (1999) 'I'll be a nothing': structure, agency and the construction of identity through assessment. British Educational Research Journal, 25, 345-354.

REID, W. A., DUVALL, E. \& EVANS, P. (2007) Relationship between assessment results and approaches to learning and studying in Year Two medical students. Med Educ, 41, 754-62.

ROEDIGER, H. L. \& KARPICKE, J. D. (2006) Test-enhanced learning: taking memory tests improves long-term retention. Psychol Sci, 17, 249-55.

RUSHTON, A. (2005) Formative assessment: a key to deep learning? Med Teach, 27, 509-513.

SCHMECK, R., RIBICH, F. \& RAMANAIAH, N. (1977) The development of a self-report inventory for assessing individual differences in learning processes. Appl. Psychol. Meas, 1, 413-431.

SCHUWIRTH, L. \& VAN DER VLEUTEN, C. (2004) Merging views on assessment. Med Educ, 38, 1208-10. 
SCHUWIRTH, L. W. \& VAN DER VLEUTEN, C. P. (2011) Programmatic assessment: From assessment of learning to assessment for learning. Med Teach, 33, 478-85.

STERN, D. T., WILliAMS, B. C., GILL, A., GRUPPEN, L. D., WOOLLISCROFT, J. O. \& GRUM, C. M. (2000) Is there a relationship between attending physicians' and residents' teaching skills and students' examination scores? Acad Med, 75, 1144-6.

TORRANCE, H. \& PRYOR, J. (1998) Investigating Formative Assessment: teaching, Learning and Assessment in the Classroom. Open University Press. Buckingham.

VAN DER VLEUTEN, C. P. \& SCHUWIRTH, L. W. (2005) Assessing professional competence: from methods to programmes. Med Educ, 39, 309-17.

VERMUNT, J. D. (1989) The interplay between internal and external regulation of learning, and the design of process-oriented instruction. Paper presented at theThird Conference of the European Association for Research on Learning and Instruction. Madrid, Spain.

VERMUNT, J. D. (1996) Metacognitive, cognitive and affective aspects of learning styles and strategies: A phenomenographic analysis. Higher Education, 31, 25-50.

VERMUNT, J. D. (1998) The regulation of constructive learning processes. Br. J. Educ. Psychol, 68, 149-171.

WENGER, E. (1998) Communities of practice Learning, meaning, and identity, Cambridge, UK, Cambridge University Press.

WOOLLISCROFT, J. O., TENHAKEN, J., SMITH, J. \& CALHOUN, J. G. (1993) Medical students' clinical self-assessments: comparisons with external measures of performance and the students' selfassessments of overall performance and effort. Acad Med, 68, 285-94. 

Chapter 2

\section{Exploring factors affecting undergraduate medical students' study strategies in the clinical years: a qualitative study}

A full text version was published as:

Al Kadri, H. M., Al-Moamary, M. S., Elzubair, M., Magzoub, M. E., Almutairi, A., Roberts, C., and van der Vleuten, C. (2011). Exploring factors affecting undergraduate medical students' study strategies in the clinical years: a qualitative study. Adv Health Sci Educ Theory Pract. 16(5):553-67 



\section{Abstract}

The aim of this study is to explore the effects of clinical supervision, and assessment characteristics on the study strategies used by undergraduate medical students during their clinical rotations. We conducted a qualitative phenomenological study at King Saud Bin Abdulaziz University for Health Sciences, College of Medicine, Riyadh, Saudi Arabia during the period from November 2007 to December 2008. We conducted semi structured focus groups interviews with students and conducted individual interviews with teachers and students to explore students' and clinical teachers' perceptions and interpretations of factors influencing students' study strategies. Data collection was continued until saturation was reached. We used Atlas-ti Computer Software (Version 5.2) to analyse the data, apply the obtained themes to the whole dataset and rearrange the data according to the themes and sub-themes. Analysis of data from interviews with twenty-eight students and thirteen clinical supervisors yielded three major themes relating to factors affecting students' study strategies: "clinical supervisors and supervision", "stress and anxiety" and "assessment". The three themes we identified played a role in students' adoption of different study strategies in the "community of clinical practice". It appeared that teachers played a key role, particularly as assessors, clinical supervisors and as a source of stress to students.

\section{Introduction}

Learning style inventories initially emphasized the relative stability of students' study strategies. They were used to predict students' future academic performance (Biggs, 1970; Biggs, 1976; Entwistle, and Entwistle, 1970; Schmeck, Ribich, and Ramanaiah, 1977). Based on the early inventories, it was found that students'study strategies reflected three basic learning models (Broadbent, 1966): surface learning, involving "repetition of analyses already carried out" and usually directed at reproducing information; deep level learning, using "a greater degree of semantic or cognitive analysis" usually aimed at gaining insight and understanding and "assessment driven category", named 
Factors affecting students' study strategies

a strategic approach to studying (Entwistle, and McCune, 2004). Further research on students' study approaches has focused more on the effect of teaching-learning environment (Biggs, 1999). It investigated the differing ways students interpret the requirements of a task within a specific learning context (Marton, and Saljo, 1997). Students' study approaches were then found to be unstable. More recent inventories have emphasized students' self-conscious reflection on studying, drawing on the ideas of "metacognition" and "self-regulation" (McKeachie, 1990; Vermunt, 1996; Vermunt, 1998). Categorizations of students' study approaches were then modified to a deep, reflective and elaborate approach and superficial with serial, reiterative, or rehearsal approach. A third approach of studying that describes methodical, wellorganized studying linked to effort and achievement motivation was added replacing the strategic approach to learning.

Several factors have been shown to influence undergraduate students' study approaches, with students adopting strategies in accordance with their interpretations of the requirements of the tasks assigned within a specific learning environment (Marton et al., 1997). These interpretations varied depending on students' perception of the academic quality of a course and the nature of the curriculum (Richardson, Dawson, Sadlo, Jenkins, and McInnes, 2007), students' implicit theories of learning on entering higher education (Edmunds, and Richardson, 2009) and the learning environment to which students are exposed (Reid, Duvall, and Evans, 2005). However, these interpretations alone do not explain the full range of students' intentions and motivations with respect to studying (Mattick, and Knight, 2009). Students' perceptions of the environment in which they learn and are assessed also interfere with their choice of the study approaches. However, it is hard to predict which choices students will make (Mattick, and Knight, 2007; Mattick et al., 2009). It appears that there is a variety of factors influencing how students tackle their academic work. These factors can lead to swings in students' appraisals of the usefulness of different study approaches. When students 
start clinical training, during which they gain experience and skills by taking part in the provision of patient care, they encounter different supervised learning environments and different assessment programs. They are also exposed to stressful situations where patients' safety is a major issue (Kennedy, Regehr, Baker, and Lingard, 2009). In this learning environment, supervisors' knowledge, skills, encouragement of a problemsolving approach, critical reflection on practice and the way they supervise students are perceived as important factors affecting students' learning and study approaches (Marrow, and Tatum, 1994).

Most assessment in the clinical workplace is directed at different levels of Miller's Pyramid (Miller, 1990). In addition to knowledge, students are assessed on their ability to perform specific skills in clinical practice. Ideally, assessment in the clinical years should focus on students' ability to organize thoughts, link theoretical knowledge to patients' management, communicate with patients and the environment and elaborate on patients' conditions. It should also focus on students' clinical skills and work. Deeplearning approaches, including knowledge integration, writing summaries and selftesting (Groves, 2005; McParland, Noble, and Livingston, 2004; Newble, and Entwistle, 1986) are important to prepare students for assessment, clinical problem solving and safe patient management. However, since students are known to swing between different study strategies in response to different environmental and contextual factors, they may occasionally use strategies involving "memorizing without understanding" (Meyer, 2000) and "fragmented knowledge" (Meyer, and Dunne, 1991).

From the above, it is clear that learning functions play a central role in the theory on regulation of learning processes (Vermunt, 1989). However, little is known about the manner in which students carry out and regulate these functions in a clinical educational context. Insight into these processes can make an important contribution to the improvement of clinical education and its instructional process. In the clinical 
Factors affecting students' study strategies

years, we expected to find a spectrum of students' responsiveness to stressful situations, in terms of study strategies, such as dealing with patient problems, assessment and clinical supervision. A question with important implications for teaching and learning then arises: under which conditions do students use which study strategies? And furthermore, which factors contribute to students' use of particular strategies? To our knowledge, no study has specifically explored this issue within the clinical learning environment. The increasing need for information on training and certification in higher education and the increasing requirements of universities to justify effectiveness and efficacy of their teaching put more importance to the answers of these questions. In this work, we have addressed two research questions: (I) what are the effects of clinical supervision, patient encounters and assessment characteristics on the study strategies adopted by medical students? and (II) can these factors be classified as promoting one of the different known study strategies?

\section{Methods}

The study was conducted at King Saud Bin Abdulaziz University for Health Sciences (KSAU-HS), College of Medicine (COM), Riyadh, Saudi Arabia between November 2007 and December 2008. The College accepts only male students and is housed within King Abdulaziz Medical City, a 900-bed tertiary care centre. KSAU-HS offers a 4 year graduate entry program with an integrated, problem-based learning (PBL) curriculum adopted from the University of Sydney.

\section{Assessment of clinical blocks}

The clinical years (years 3 and 4) of the Undergraduate Medical Program consist of five different clinical blocks. Within each clinical block, students rotate in different clinical attachments and are assigned to supervisors responsible for clinical training and assessment. The term "clinical supervisor" refers to 
experienced clinicians, who implement the curriculum objectives and are responsible for patients' protection and safety while providing professional support and teaching to students. Their role also includes helping individual students to develop knowledge and competence and assume responsibility for their own practices.

At KSAU-HS, students accompany their clinical supervisors throughout their daily clinical activities and the supervisors assign students tasks that are in alignment with the curriculum objectives. These tasks are performed under direct supervision of supervisors or senior team members, as students are not allowed to conduct clinical encounters unsupervised. When students rotate to another clinical attachment within the same block, they change supervisors and clinical exposure. The assessment program for the clinical years is block-based. At the end of each clinical attachment, students meet with their clinical supervisor for verbal and written qualitative formative assessment and feedback. Clinical supervisors are responsible not only for formative but also for summative assessments, both of which should reflect students' performance during the clinical attachment. In this paper, we use the term "summative assessment" with reference to assessment contributing to course grades, while "formative assessment" refers to assessment as a tool to aid the learning of students but without grading. During each clinical block, students are given one summative long case exam and one mid-block summative multiple choice (MCQ) exam, while the end-of-block assessment consists of a summative MCQ exam, a summative objective structured clinical examination (OSCE) and a summative MCQ exam on data interpretation. The MCQ exams are mostly composed of type A questions aimed at assessing the "knows how" level of Miller's pyramid. Assessment of students' clinical performance during all attachments within a block counts for $40 \%$ of the final block grade, while the final exam is worth the remaining $60 \%$. 
Factors affecting students' study strategies

\section{Participants}

We conducted semi-structured interviews (Ashworth, 2000; Creswell, and Miller, 2000; Ritchie, and Spencer, 1994) because this type of interview provides more freedom to obtain a complete picture of participants' experiences. By way of purposeful sampling, we invited students who had experienced the clinical blocks and the related assessments and the clinical supervisors of the different blocks to participate in the study. We performed seven semi-structured focus group interviews in groups of four students to achieve greater variation in the discussion and elaboration on students' lived experiences. Occasional conflicts among students enriched the data, which led to deeper discussions and more varied opinions. The participants were 28 out of the total of 61 students in the third and fourth years of the study program. We also conducted semi-structured individual interviews with 13 out of 44 clinical supervisors. The thirteen clinical supervisors were randomly selected from the pool of clinical supervisors who all agreed to participate. The purpose of using individual interviews was to allow the teachers to speak openly and without restrictions or bias. Open-ended interview questions similar to those used with the students were used to probe the teachers' views on students' study strategies and the factors which influenced their strategy selection (Appendix). Interview schedules included some open core questions and a number of suggestions for continuation questions. Questions were asked about the methods used by the students when studying the course materials, the role of the teachers in their studies, the factors affecting the way the students' study, views on studying at the university, study expectations and study plans and the supervisors' roles in patients' encounters and assessment. For triangulations of the data, we conducted a second set of interviews with all of the thirteen clinical teachers and twelve of the students who had participated in the focus groups. They were asked about their opinions on the results of the first round of students' focus group interviews (figure 1). 


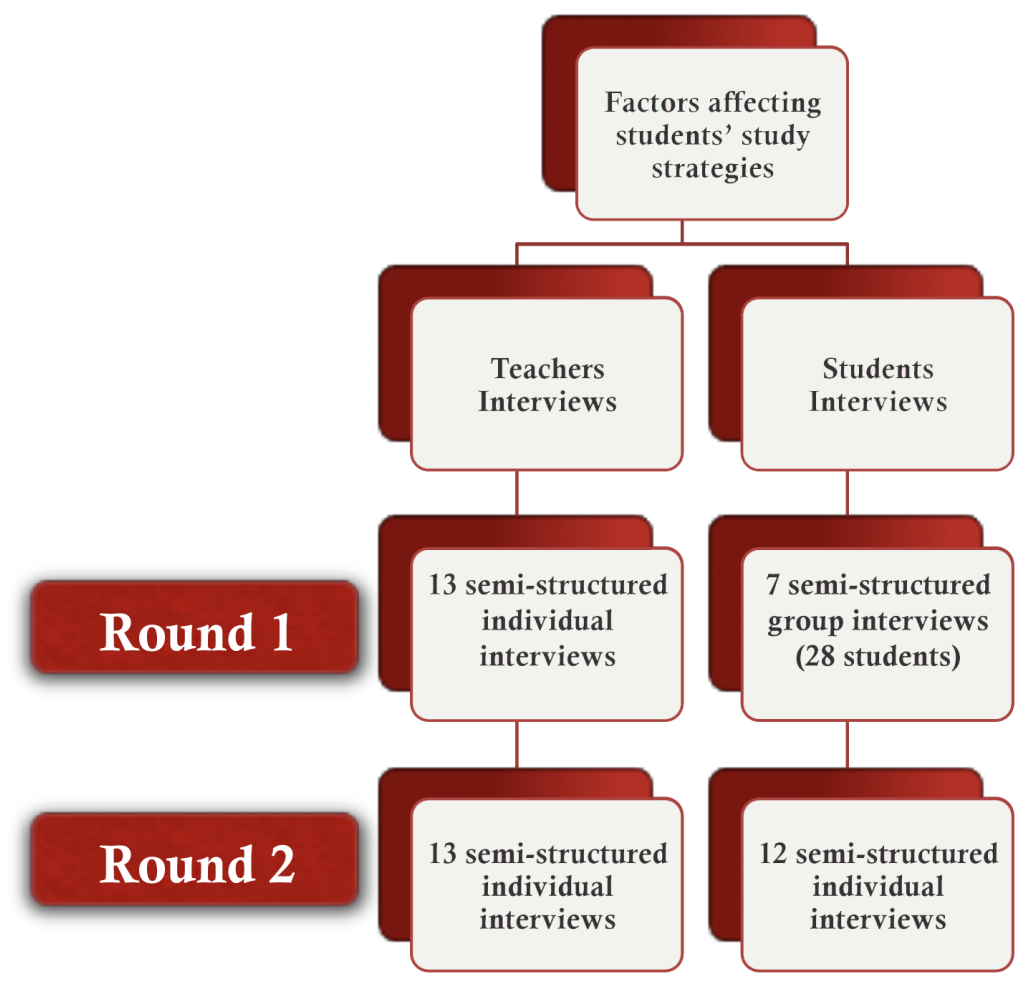

Figure 1: Diagram representing the two rounds of the research conduction

Students' reported study approaches and related factors were the focus of these interviews. The themes derived from the initial analysis were used to create further interview questions for a more in-depth individual exploration of the reasons for the students' lived experiences. The KSAU-HS, COM Research Ethics Committee approved the study.

\section{Data collection}

All interviews were conducted by the principal investigator and a research assistant. Both students and teachers were allowed to talk freely and express their opinion on the effects of clinical supervision, patient encounters and assessment characteristics on 
Factors affecting students' study strategies

students' adopted study strategies. We appreciated the presence of complex relationships between students' different study strategies and their teaching and learning environment; therefore, we decided to choose the phenomenographical approach. Phenomenography is a research methodology that can be used to map the qualitative different ways people experience, conceptualize, perceive and understand phenomena (Marton, 1986). The objective of this method is to frame and describe these qualitative differences in conceptual categories. The phenomenographic approach has been used in various educational research contexts and with various populations (Marton, 1986; Pramling, 1990; Vermunt, 1996). We examined qualitatively the different ways by which students approach their studying in response to different stressful conditions. Our assumption was that different students perceive clinical exposure and direct patients encounter, clinical assessment, supervisors and supervision differently (Marton, 1986). We aimed to understand the similarities and differences between students' perceptions (Severiens, and Ten Dam, 1997) and the relationship between these differences and students' learning strategies. The themes of the interviews and focus groups were summarized and presented to the participants for feedback and reactions. The researchers' field notes, debriefing notes and the verbatim transcriptions of all the interviews and focus groups were integrated. This process was repeated for the second set of interviews. Each focus group session lasted 45-90 min and the individual interviews lasted for $30-45 \mathrm{~min}$.

\section{Data analysis}

All interviews were transcribed verbatim and read completely. Subsequently, the principal investigator studied the interviews several times. Themes and sub-themes relevant to the research questions were assigned and representing quotes were selected. This process was repeated for each interview in order to capture the full breadth and diversity of the students' and teachers' views and experiences (Ritchie et al., 1994). Similarities and differences were determined. Themes and sub-themes (codes) were 
then refined and finalized. In order to improve the credibility and transferability of the data, we used member checking whereby two of the authors, HK and MM, compared the themes and codes resulting from the analyses of 3 students' and 3 teachers' interviews and discussed differences until they reached a consensus (Creswell et al., 2000; Polit, and Beck, 2003). Occasionally, students elaborated on their experiences during the pre clinical years (phase I) or even their previous study. The data was considered as confounder and was not included in the research. The Computer Software Atlas-ti (Version 5.2) was used to apply the themes to the whole dataset and to rearrange the data according to themes and subthemes.

\section{Results}

We invited 56 out of a total of 61 students (clinical years 3 and 4) to participate in the study. Twenty-eight of these students participated voluntarily (50\% response rate). The students' mean age was (26.74) years, and their mean graduation GPA was (3.96/5). These characteristics are similar to the mean age (26.77 years) and the mean GPA at the graduation time $(3.89 / 5)$ of the non participant students. The analysis revealed three major themes in relation to factors affecting students' study strategies: "clinical supervisors and supervision", "stress and anxiety" and "assessment" (table 1). We presented the themes with illustrative quotes from interviews with students (S) and teachers $(\mathrm{T})$.

\section{Clinical supervisors and supervision in a clinical context}

Students indicated that their learning strategies were strongly influenced by supervision, particularly when they felt it was constructive. Students appreciated effective workplace supervision, and indicated that it had a positive impact on their study strategies. Constructive supervision helped students to integrate clinical knowledge into clinical practice, summarize patients' histories and solve patients' problems. Teachers as role models, their way of coaching and guiding students, their experience and their 
Factors affecting students' study strategies

commitment to clinical teaching were identified as crucial factors in enhancing the quality of learning in the workplace. According to the students, there was a relationship between students' motivation, teaching and study strategies. Direct supervision was a strong motivator for students to use deep learning strategies. Supervisors who showed a flexible attitude towards students and their knowledge stimulated students to use a focused study strategy and a deeper approach to learning. The availability of experienced and motivated supervisors, who support direct patients' encounter made students more interested in critically analyzing patients' clinical conditions, read about them and utilize this work to formulate management plans. Some of the students related the way they tackled their patients' clinical problems to their supervisors, saying, "It depends on whom I rotate with, who my supervisor is and who assesses me,... this may encourage me to study and read more" and "...to show me how to read, what to follow and.... then.. ok he can... he can say go by yourself..." (S). Thus, teachers' availability and teaching experience were important factors in the selection of students' study approach. According to the teachers, their workload needed to be redistributed to make them available for direct supervision. The role of the college was emphasized in this regard. Teachers felt that a good balance between clinical work and teaching encouraged them to involve students in patient management and increased students' interest in clinical training "more time spent with patients, makes them keen to learn" (T). Due to the conflict between clinical teachers' assignment as clinicians and their duties as clinical teachers, their work arrangement appears to be difficult to achieve. Teachers recommended that all their clinical and academic assignments come from one direction. "The college should assign teachers' workload and not the clinical departments" (T).

Students reported that patient encounters boosted their confidence in performing physical examinations and led to gains in clinical experience. Direct contact with patients stimulated the students to approach their learning deeply. They were more 
capable of integrating their theoretical knowledge into clinical practice and elaborating on their patients" conditions. The studied teachers have supported the same idea, "The most important trigger for students to learn is their new experience in the hospital. The resulting excitement...stimulates them to do more reading and preparation for assessment", (T) and "clinical attachment, the more cases I see the better. I go home and read about the disease and try to gather all relevant information about it" (S).

When supervision was disorganized and not constructive, many clinical activities of the students went unsupervised and students became frustrated and lost interest in clinical training. This had a negative effect on their studies. Students in this case opted to utilize the reiterative and rehearsal method for reading. They read from lecture slides and previous students' notes. The unavailability of supervisors' time and motivation led to their superficial approach to learning. "How can he assess our efforts? He didn't show up and he did not see us at all...I would rather stay at home and read..." (S). It was difficult for teachers to find time for teaching in their busy clinical schedules. As a result, the amount of time devoted to teaching depended mainly on teachers' motivation and willingness to teach. Variability in attention of supervisors led to variability in students' approaches to learning. Students' narratives were consistent with a deep approach to their learning in the presence of increased systematic clinical supervision. "...we work in a busy department; we don't have much time assigned to students...", (T) and "... there are tutors who love to teach and devote time to teaching and some just don't", (T) and "Yes, we usually focus during the clinical attachments and make a real effort to learn from our supervisors. Their presence gives us a lot of motivation to use the time dedicated for the clinical attachment to learn. But some supervisors don't show up, arrive late or leave immediately after rounds ... ", (S).

Teachers became more interested in teaching when they identified teaching competencies that needed to be improved. They saw faculty development activities to 
Factors affecting students' study strategies

improve their teaching skills as major factors, which could indirectly improve students' study strategies “ "...to improve student learning, supervisors” teaching skills should be improved first" (T).

\section{Stress and anxiety}

Students indicated that supervisors could be a potential source of stress and anxiety; for instance when supervisors over-estimated their clinical abilities, asking them to answer questions or perform tasks that went beyond the curriculum objectives. This was even more stressful when it happened in front of a patient or a colleague. Stress and anxiety led to intermittent, unfocused reading and a superficial approach to learning. Occasional unavailability of supervisors at the workplace was another source of stress for students, especially since the students were dependent on supervisors for their final marks. Students felt it was unfair to be assessed by someone who was not quite familiar with their performance. To avoid stress and embarrassment, students start to speculate on the possible tasks or questions that they might be asked by their supervisors and try to prepare for them. This results in rehearsal of sporadic and disorganized reading. ' Our supervisors should orient their teaching to the curriculum objectives. They should be familiar with the required levels. I think some of them expect us to perform at the level of a resident" (S) and "we have to read for the exam, we have to read for the clinical attachment, this is the problem..., how can I get time in between to read, I am always under stress..." (S) and "how can I meet the entire objectives in three months? So we are under stress. I tried to read from here and there... If without stress, ... I can organize myself; schedule my topics, my objectives ..." (S).

\section{Assessment}

Opinions about the impact of different assessment methods (OSCE, long case, etc.) on students' study strategies differed. Some students said that assessment methods did not affect how they prepared for exams, but some other students said they were affected by 
assessment methods, "I will be prepared regardless of the exam method" (S) and "The assessment method affects how I study" (S). Students' opinions differed from those of the majority of the teachers. Teachers thought that all students were exam oriented and used assessment strategically to achieve their goals.

Some students mentioned that they modified their study strategies based on the weighting of the subject and how much time was assigned for reading "......as students, we organize our study agenda based on the exam schedule and assessment methods" (S). As a result, students sometimes adopted superficial strategies trying to read all possible

Table 1: Various themes and codes relating to factors affecting students' study strategies in a clinical context

\section{Themes}

Codes

\section{Clinical supervisors and supervision}

Constructive supervision

Role modeling

Teaching time

Teaching experience

Teachers motivation

Faculty development

Stress and anxiety

Over estimation of students objectives

Supervisors un-availability

\section{Assessment}

Assessment method

Assessment weight

Assessment time

Fair assessment 
Factors affecting students' study strategies

information that might come in the exam without relating it to a patients' management plan. They opt to guarantee a pass or even high marks by mimicking what looks like an achievement motivation study strategy "... we are doing surgery, I have consulted my friends ... and I know some interns. I selected the best book on surgery and read it from a to $z$. I read each day ten pages till I covered it all ..." (S). Despite these differences, there was congruence between students and teachers in their identification of exam marks as a main influence on study strategies. Students acted as "mark hunters" using variable strategies (deep, superficial, and to a higher extent an effort management strategy). They aimed at passing the exam or scoring high marks "I just work hard because I just want to get an $A$ " (S).

The sub-theme of a "fair assessment" was derived from views expressed by both students and teachers. It was described as a well-designed assessment aimed at students' true level of training and in alignment with curriculum objectives. It gave students a sense of security and was an important factor in encouraging them to study deeply. Properly designed blue printing and linkage of assessment to the curriculum objectives was one of the characteristics (of fair assessment), "final exam should reflect all the curriculum components in a fair distribution percentagewise" (T) and "if assessment items are not too precise, it's difficult for assessors to decide on the mark, is it one or two, two or three, one or zero" (T). Assessment that reflects curriculum objectives promotes students' satisfaction and affects students' study approach. It guides students while studying based on these objectives. However, that will be towards effort management strategy. Hence, the way we structure our curriculum objectives will have a major effect on students' study approach "I follow the objectives, ... why I follow them?.... because I always think about the final exam." (S). 


\section{Discussion}

In the clinical context, students' knowledge about their training requirements, their assessment and their supervision created a common ground for students' learning, guided their study strategies and gave meaning to their actions and interactions. The presence of a community of students and supervisors in the clinical context created the social fabric of students' learning, relations with the surroundings and their interrelations.

The term "community of practice" was first described by Lave and Wenger (1990) as learning through practice and participation. It describes the function of a group of people who have a variety of experiences but share an interest or a profession. They share information and experiences, learn from each other, and have similar opportunities to develop (Lave, and Wenger, 1990). Everybody is involved in a number of communities of practice whether at work, school, or home. Their structural characteristics are defined as a domain of knowledge, a notion of community and a practice (Wenger, 1998).

It appears that the studied students' learning in the clinical context and their social experiences are a new example of "community of practice" that can be called "community of clinical practice". In this research, we have evaluated students' actions and interactions in the "community of clinical practice" and their effect on their study strategies. We then reached in our evaluation Kirkpatrick's second level of learning evaluation model (Kirkpatrick, 1994) (Figure 2). In the "community of clinical practice", students are brought together by joining common clinical and educational activities and by what they have learned through their mutual engagement in these activities (Wenger, 1998). In this context, students had to generate and prepare a shared repertoire of ideas and commitments. They had to share ways of doing and approaching tasks. The studied students" ongoing activities and their involvement in "community of clinical practice" manipulated their learning strategy approach (Lave, and Chaiklin, 1993). Students' clinical training resulting stress, clinical supervisors and supervision 
Factors affecting students' study strategies

and the implemented assessment were identified as factors affecting students' study strategies. Depending on whether these factors have a negative or positive impact on the learning environment, students' study strategies can become increasingly surface oriented, swing between surface, deep, effort and achievement motivation or reflect a deep learning strategy (Figure 2). This is consistent with evidence in both medical and non-medical disciplines (Groves, 2005; Ramsden, and Entwistle, 1981; Schmidt, CohenSchotanus, van der Molen, Splinter, Bulte, and Holdrinet, 2010; Seabrook, 2003).

A teacher's personality, availability and effectiveness as a role model in the "community of clinical practice" influenced how students studied. On the whole, the supervisory practice of clinical teachers strongly influenced the way students studied. Students placed great value on the availability of supervisors to provide guidance and coaching. While availability enhanced skill acquisition, lack of supervision deprived students of essential educational support, thereby creating a potential source of stress. Findings from other studies lend support to factors identified by the teachers and students in this study. The absence of supervisors can also lead to a loss of the effect of role modelling (Kennedy et al., 2009) and can increase the likelihood of students practising surface or strategic approaches to learning (Gray, Hildreth, Fisher, Brown, Jones, Turner, and Boobis, 2008).

Teachers' methods of presenting information to students, their supervisory skills (Biggs, 1999), their skills in activating clinical reasoning and their ability to motivate students have all been identified as major factors in determining students' study strategies (Diemers, Dolmans, Verwijnen, Heineman, and Scherpbier, 2008; Entwistle et al., 2004). Furthermore, well trained teachers have been shown to be more interested in direct supervision during patient encounters (Diemers et al., 2008). Here lies the importance of teachers' professional development, which can lead to more competent, satisfied, motivated and reliable teachers (Bland, Seaquist, Pacala, Center, and Finstad, 2002). It has been shown that properly trained teachers are better equipped to provide 
less structured flexible clinical teaching. This facilitates students' transition from preclinical to clinical training and its related "community of clinical practice" issues, motivates the students to learn and helps them to modify their professional behaviour through utilizing their teachers' feedback (Diemers et al., 2008; Fry, 1993).

Our results with regard to available time for teaching are also confirmed by other studies, which have reported variations between institutions and teachers in the acceptance of responsibility for clinical teaching and the time allocated for supervision (Hayes, 2008; Wimmers, Schmidt, and Splinter, 2006). In our study, limited time for clinical teaching was regarded as a barrier to high quality teaching practice, with some of the teachers saying they needed protected time for teaching while others were not prepared to allocate more time for teaching. The variation in teachers' perception of time needed to transmit knowledge and practice in the domain of "community of clinical practice" reflects their variation in interest, interaction and sense of responsibility.

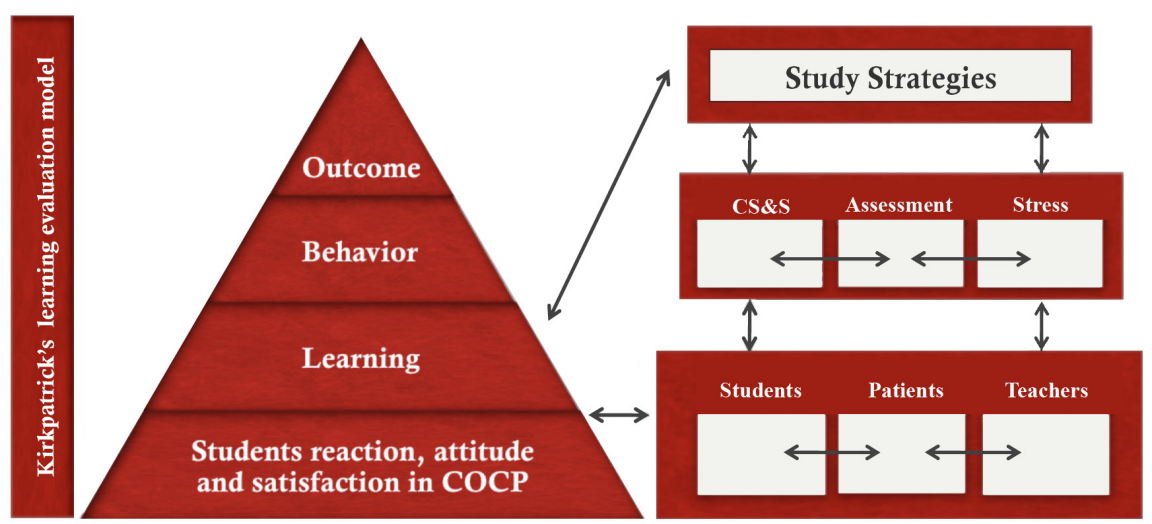

Figure 2: Evaluation of factors affecting students' study strategies in the "community of clinical practice".

COCP Community of clinical practice, CS\&S Clinical supervisors and supervision 
Due to factors in the setting of our study, the results offer no insights into the role of supervisors in ensuring patient safety and alleviating students' stress. Because the students were not allowed to engage in patient contacts without supervision, stress related to unsupervised patient encounters and its effects on study strategies could not be studied (Kennedy et al., 2009). However, there were other types of stress experienced by students, notably stress related to teachers combining the supervisory role with responsibility for assessment. This interaction promoted patchy reading and surface or strategic plans to the detriment of deep study strategies, thereby creating a hidden curriculum (Al Kadri, Al-Moamary, and van der Vleuten, 2009).

Most students and teachers perceived assessment as a major influence on the way students prepared for exams. This is not surprising based on the generally accepted view that clinical exams should be more than tests of factual knowledge. Students need good knowledge as well as the ability to apply that knowledge to any given task or clinical scenario. Encouragement of appropriate application of clinical knowledge can foster a deep approach to learning and enhance performance on clinical assessments (Norton, 2004). Timing and weight of exams influenced students' study strategies, which confirm that students use deep, strategic and surface approaches variably, depending on perceived challenges of the learning environment. Similar variation was reported in other studies (Groves, 2005; Papinczak, 2009).

There are several limitations to this study. This research has evaluated the first two levels of Kirkpatrick's learning and training evaluation (students' reaction and their learning in the clinical context). To evaluate the third and fourth levels (Kirkpatrick, 1994; Phillips, 1996), further research is required. This will enable us to understand the continuous interaction among students, teachers, education environment and patients' care and satisfaction. Moreover, the results may be uniquely applicable to the medical 
school where it was conducted. However, by including the main stakeholders and using a clear methodology we aimed to give a clear unbiased example on students' learning behavior in one of the "communities of clinical practice". To assess different behave our in different environments, and different genders further research is needed. Some might raise the question whether it would not have been preferable to explore learning strategies in specific clinical specialties rather than across all clinical blocks. They may be right in arguing that factors observed in one context may be different in another one. We think this should be a subject for further research. Moreover, our study design was limited to students' and teachers' views and experiences and did not include the relationship between learning strategies and students' outcomes. A relatively recent study from the Netherlands found no significant relationships between learning strategies and clinical performance (van Lohuizen, Kuks, van Hell, Raat, and CohenSchotanus, 2009), but we acknowledge that this is an important area for further work.

\section{Conclusion}

In an educational clinical context, it was found that students' social interactions affect their studying approach. This social and clinical education environment represents a new example of the community of practice theory, we named it: "community of clinical practice". In this community, we identified factors with potential negative and/or positive effects on the likelihood of students adopting different learning strategies. In the community of clinical practice, teachers played a crucial role, particularly in assessment and clinical supervision and can be a potential source of stress to students. They were unaware of the potential negative and positive consequences of their actions as supervisors and assessors with regard to students' learning strategies. Awareness enhancement through an effective faculty training program is required to promote desired students' study strategies. Further promotion of desired study strategies can be obtained through clinical curriculum modification. The emphasis should be on more supervisors and supervision role, more clinical involvement, more clinical assessment and constructive alignment. 
Factors affecting students' study strategies

Appendix: Factors affecting students' study strategies

Semi-structured interview questions

1. Can you please describe to me which block or rotation you are currently in?

2. What are the clinical activities you are expected to perform in this block?

3. In addition to this, what other structured learning activities do you take part in?

4. How do you usually study? What factors may affect (change) your way of studying? Why?

5. What do you think of your clinical supervision? What about your supervisors? What about your team? How did this affect your studying? So what improvements might make you focus more on your patients' management?

6. What is your assignment within the clinical team? How do you tackle your patients' problems?

7. What about stress when you started the clinical training? How do you deal with it, if any? Will this affect your studying?

8. What assessments have you undergone so far this year?

9. So how do you prepare for these various assessment activities? Do you use different strategies (ways) for different assessments? Can you give me an example of the strategies (ways) that you would use?

10. What kind of things influence what you read or do in preparation for the various assessments?

11. What do you think of your assessment? What do you think of its fairness?

12. What about the alignment or link between the structured teaching and learning program and assessment? How will this affect your studying or the way you study?

13. What do you think of the timing of your assessments? What about the different weightings given to each? Does this affect the way you study? 


\section{References}

AL KADRI, H. M., AL-MOAMARY, M. S. \& VAN DER VLEUTEN, C. (2009). Students' and teachers' perceptions of clinical assessment program: A qualitative study in a PBL curriculum. BMC Res Notes $2,263$.

ASHWORTH, P. (2000). Achieving Empathy and Engagement: a practical approach to the design, conduct and reporting of phenomenographic research. Studies in Higher Education 25, 295-308.

BIGGS, J. (1999). Teaching for Quality Learning at University. SRHE and Open University Press, Buckingham.

BIGGS, J. B. (1970). Faculty pattern in study behaviour. Aust. J. Psychol 22, 161-174.

BIGGS, J. B. (1976). Dimensions of study behaviour: Another look at a.t.i. Br. J. Educ. Psychol 46, 6880.

BLAND, C. J., SEAQUIST, E., PACALA, J. T., CENTER, B. \& FINSTAD, D. (2002). One school's strategy to assess and improve the vitality of its faculty. Acad Med 77, 368-76.

BROADBENT, D. E. (1966). The well-ordered mind. Am. Educ. Res. J 3 281-295.

CRESWELL, J. W., MILLER, D. L. . (2000). Determining validity in qualitative inquiry. Theory Into Practice 39, 124-130.

DIEMERS, A. D., DOLMANS, D. H., VERWIJNEN, M. G., HEINEMAN, E.\& SCHERPBIER, A. J. (2008). Students' opinions about the effects of preclinical patient contacts on their learning. Adv Health Sci Educ Theory Pract 13, 633-47.

EDMUNDS, R. \& RICHARDSON, J. T. (2009). Conceptions of learning, approaches to studying and personal development in UK higher education. Br J Educ Psychol 79, 295-309.

ENTWISTLE, N.\& MCCUNE, V. (2004). The Conceptual Bases of Study Strategy Inventories. Educational Psychology Review 16, 325-345.

ENTWISTLE, N. J. \& ENTWISTLE, D. M. (1970). The relationships between personality, study methods and academic performance. Br. J. Educ. Psychol 40 132-141.

FRY, A. W. (1993). Capturing the complexity of clinical learning environments with multiple qualitative methods. Evaluation and the Health Professions 16, 44-60.

GRAY, C. S., HILDRETH, A. J., FISHER, C., BROWN, A., JONES, A., TURNER, R. \& BOOBIS, L. (2008). Towards a Formative Assessment of Classroom Competencies (FACCs) for postgraduate medical trainees. BMC Med Educ 8, 61.

GROVES, M. (2005). Problem-based learning and learning approach: is there a relationship? Adv Health Sci Educ Theory Pract 10, 315-26.

HAYES, R. (2008). Assessment in medical education: Roles for clinical teachers. The Clinical Teacher 5, 23-27.

KENNEDY, T. J., REGEHR, G., BAKER, G. R. \& LINGARD, L. A. (2009). 'It's a cultural expectation...' The pressure on medical trainees to work independently in clinical practice. Med Educ 43, 645-53.

KIRKPATRICK, D. L. (1994). Evaluating Training Programs. Berrett-Koehler Publishers, Inc, San Francisco.

LAVE, J. \& WENGER, E. (1990). Situated Learning: Legitimate Periperal Participation. Cambridge University Press, Cambridge, UK.

LAVE, J., AND CHAIKLIN, S. (1993). Understanding Practice: Perspectives on Activity and Context. 
University of Cambridge Press, Cambridge.

MARROW, C. E. \& TATUM, S. (1994). Student supervision: myth or reality? J Adv Nurs 19, 1247-55. MARTON, F. (1986). Phenomenography - A research approach investigating different understandings of reality. Journal of Thought 21, 28-49.

MARTON, F. \& SALJO, R. (1997). Approaches to learning. In The Experience of Learning, 2nd ed. (MARTON, F., HOUNSELL, D. J. \& ENTWISTLE, N. J., eds.), pp. 39-58. Scottish Academic, Edinburgh, UK.

MATTICK, K. \& KNIGHT, L. (2007). High-quality learning: harder to achieve than we think? Med Educ 41, 638-44.

MATTICK, K. \& KNIGHT, L. (2009). The importance of vocational and social aspects of approaches to learning for medical students. Adv Health Sci Educ Theory Pract 14, 629-44.

MCKEACHIE, W. J. (1990). Research on college teaching: The historical background. J. Educ. Psychol 82, 189-200.

MCPARLAND, M., NOBLE, L. M. \& LIVINGSTON, G. (2004). The effectiveness of problem-based learning compared to traditional teaching in undergraduate psychiatry. Med Educ 38, 859-67.

MEYER, J. H. \& DUNNE, T. T. (1991). Study approaches of nursing students: effects of an extended clinical context. Med Educ 25, 497-516.

MEYER, J. H. (2000). Variation in contrasting forms of 'memorising' and associated observables. $\mathrm{Br} J$ Educ Psychol 70 ( Pt 2), 163-76.

MILLER, G. E. (1990). The assessment of clinical skills/competence/performance. Academic Medicine 65, 63-67.

NEWBLE, D. \& ENTWISTLE, N. (1986). Learning styles and approaches: implications for medical education. Med Educ 3, 162-175.

NORTON, L. (2004). Using assessment criteria as learning criteria: a case study in psychology. Assessment and Evaluation in higher education 29, 687-702.

PAPINCZAK, T. (2009). Are deep strategic learners better suited to PBL? A preliminary study. $A d v$ Health Sci Educ Theory Pract 14, 337-53.

PHILLIPS, J. (1996). How much is the training worth? Training and Development 50, 20-24.

POLIT, D. F. \& BECK, C. T. (2003). Analyzing Qualitative data. In Nursing Research: Priciples and Methods, pp. 430-439. Lipincott, Philadelphia.

PRAMLING, I. (1990). Learning to learn: a study of Swedish preschool children. Springer, New York. RAMSDEN, P. \& ENTWISTLE, N. J. (1981). Effects of academic departments on students' approaches to studying. British Journal of Educational Psychology Review 51, 368-383.

REID, W. A., DUVALL, E. \& EVANS, P. (2005). Can we influence medical students' approaches to learning? Med Teach 27, 401-407.

RICHARDSON, J. T., DAWSON, L., SADLO, G., JENKINS, V. \& MCINNES, J. (2007). Perceived academic quality and approaches to studying in the health professions. Med Teach 29, e108-16.

RITCHIE, J. \& SPENCER, L. (1994). Qualitative data analysis for applied policy research. In Analyzing qualitative data (Bryman, A., and Burgess, R., eds.). Routledge, London.

SCHMECK, R., RIBICH, F. \& RAMANAIAH, N. (1977). The development of a self-report inventory for assessing individual differences in learning processes. Appl. Psychol. Meas 1, 413-431.

Schmidt, H. G., Cohen-Schotanus, J., van der Molen, H. T., Splinter, T. A. W., Bulte, J. \& Holdrinet, R. 1. 
(2010). Learning more by ebting taught less: A "time-for-self-study" theory explaining curricular effects on graduation rate and study duration. Higher Education 60, 287-300.

SEABROOK, M. A. (2003). Medical teachers' concerns about the clinical teaching context. Med Educ 37, 213-22.

SEVERIENS, S. E. \& TEN DAM, G. T. M. (1997). Gender and gender identity differences in learning styles. Educ. Psychol 17, 79-93.

Van Lohuizen, M. T., Kuks, J. B., van Hell, E. A., Raat, A. N. \& Cohen-Schotanus, J. (2009). Learning strategies during clerkships and their effects on clinical performance. Med Teach 31, e494-9.

VERMUNT, J. D. (1989). The interplay between internal and external regulation of learning, and the design of process-oriented instruction, Madrid, Spain.

VERMUNT, J. D. (1996). Metacognitive, cognitive and affective aspects of learning styles and strategies: A phenomenographic analysis. Higher Education 31, 25-50.

VERMUNT, J. D. (1998). The regulation of constructive learning processes. Br. J. Educ. Psychol 68, 149-171.

WENGER, E. (1998). Communities of practice Learning, meaning, and identity. Cambridge University Press, Cambridge, UK.

WIMMERS, P. F., SCHMIDT, H. G. \& SPLINTER, T. A. (2006). Influence of clerkship experiences on clinical competence. Med Educ 40, 450-8. 

Chapter 3

\section{Students' and teachers' perceptions of clinical assessment program: A qualitative study in a PBL curriculum}

A full text version was published as:

Al Kadri, H. M., Al-Moamary, M. S., and van der Vleuten, C. (2009). Students' and teachers' perceptions of clinical assessment program: A qualitative study in a PBL curriculum. BMC Res Notes 2, 263. 



\section{Abstract}

Background: "Examinations drive students' learning." This statement refers to what is assumed to be one of the strongest relationships in education. We explored in this research how and why students differ in their approaches to learning, how assessment affects deep learning, and which barriers stand in the way of good assessment and learning in the clinical years of a Problem Based Learning (PBL) graduate entry medical curriculum.

Findings: Method: We conducted a qualitative, phenomenological study using semi structured group interviews with students and semi-structured individual interviews with teachers and students. The transcripts were analyzed, and themes were identified.

Setting: The research was conducted at the King Saud bin Abdulaziz University for Health Sciences, College of Medicine, Riyadh, Saudi Arabia from November 2007 to March 2008.

Results: A total of 28 students participated in 7 focus group interviews. Semi-structured individual interviews were conducted with 12 teachers and 12 students. The analysis yielded four themes: summative assessment, formative assessment, continuous assessment of clinical attachments, and learning objectives.

Conclusions: The results of this study confirm that assessment affects students' perceptions of learning and how they learn. These effects are not uniformly positive. According to the students, the predominantly summative assessment program offers little inducement to engage in deep learning. They express a clear preference for formative assessment, which may foster a deeper approach to learning. Efforts to achieve more clinically relevant assessment with adequate balance between the various types of assessment are required. Research is needed to decide this balance. 


\section{Introduction}

The concept that assessment drives learning has been accepted as one of the principles of good assessment practice (Messick, 1995). Assessment affects not only what students learn but also how they learn (Norton, 2004). Unfortunately, some student learning strategies contribute little to the learning processes intended by the curriculum (Norton, 2004).

The relationship between assessment and the learning behaviors of medical students has been examined by several authors (Larsen, Butler, and Roediger, 2008; McDaniel, Roediger, and McDermott, 2007). In examining this relationship, Biggs focused on the concept of constructive alignment (Biggs, 2003), advocating an approach to curriculum development that ensures that both teaching and assessment are aligned with the curriculum's stated learning objectives.

Different effects of assessment on learning have been reported in the literature. Some studies have shown that students' approaches to learning and their retention of knowledge differ across a range of assessment methods (Larsen et al., 2008; Reid, Duvall, and Evans, 2007). In a quantitative study, Gijbels et al (Gijbels, Segers, and Struyf, 2008) found a relationship between the learning environment, assessment demands as perceived by students, and students' approaches to learning; however, these findings require further clarification. A study by Baeten et al (Baeten, Dochy, and Struyven, 2008) found no evidence that assessment advanced a deep rather than a superficial approach to learning. Mattick et al (Mattick, and Knight, 2007) emphasized the importance of understanding the barriers to a deep learning approach as perceived by students.

In summary, if we want to enhance the students' learning and promote deep learning, we need to understand how and why students differ in their approaches to learning, how assessment affects deep learning, and which barriers stand in the way of good assessment and learning. In this study, we explored these issues by seeking students' 
and teachers' perceptions of their experiences with the assessment program in the clinical years of a PBL curriculum.

\section{Methods}

We conducted a phenomenological study to examine what happens when students direct their study strategies to fit with assessment rather than learning objectives. Our aim was to gain insight into how students and teachers experienced the assessment program. We explored students' perceptions in semi-structured group interviews. When more depth into students' perceptions was required, we conducted semi-structured individual interviews with students until we reached data saturation. Semi-structured individual interviews were also performed to explore the perceptions of teachers.

\section{Study setting}

The study was conducted at King Saud Bin Abdulaziz University for Health Sciences (KSAU-HS), College of Medicine (COM), Riyadh, Saudi Arabia in the period from November 2007 to March 2008. The college accepts only male students and is housed within King Abdulaziz Medical City, a 900-bed tertiary care center. The KSAU-HS, COM curriculum is a Problem-Based Learning (PBL) curriculum. It is a four-year graduate entry program consisting of two preclinical years and two years of clinical education. During the clinical years, there are two concurrent but different paths of learning, one of workplace learning and one of PBL group discussions.

\section{Assessment program}

The assessment program for the clinical years at KSAU-HS, COM is block-based (total of five blocks). In each block, students' assessment is divided into two main parts (figure 1). Assessment of students' performance during each block clinical attachments accounts for $40 \%$ of the final grade (students portfolio), and the final examination accounts for the other $60 \%$. By the end of each clinical attachment 
(every 1-4 weeks), students meet with their clinical supervisors and are expected to receive a written qualitative formative assessment and feed back on their performance during that attachment. In the present paper the term "summative assessment" refers to an assessment performed to assign students a course grade, "formative assessment" refers to an assessment as an educational tool to aid students' learning without grading, and "continuous assessment" refers to an assessment of students' progress based on work they do or tests they take throughout the block.

\section{Study population}

Students were recruited by a stepwise purposeful sampling approach. 56 out of a total of 61 students who had experienced the assessment program of the clinical years were invited to participate in the study. 28 students participated in seven semi-structured focused group interviews. Each group interview was attended by four students. Purposeful sampling was also used to select 12 teachers out of the pool of clinical years block directors and clinical supervisors. All teachers who were contacted agreed to participate and were interviewed individually to allow more freedom and avoid bias. To explore the obtained themes, 12 additional students out of the same pool were individually interviewed. All interviews were done by the principal investigator, who was at a comparable seniority with the interviewed teachers and had not yet started teaching any of the interviewed students at the time of data collection.

\section{Data collection}

Students were asked to talk about their perceptions of the assessments and how their experiences with the assessment program affected their learning activities and strategies for handling assessments. Each group interview lasted about 40-75 minutes. We benefited from some of the conflicting opinions that were expressed by the students. When no new themes arose, we stopped conducting interviews. The interviews were recorded on tape, and students' nonverbal behavior was registered. The audiotapes 
were transcribed verbatim, and field notes and the verbatim transcriptions of the group interviews were integrated. This process was repeated for the individual semi-structured interviews with the students (12 students took part in interviews lasting 30-45 minutes) and teachers (12 teachers took part in interviews lasting 30-45 minutes).

\section{Data analysis}

The transcriptions of the interviews were analyzed using Atlas-ti (Version 5.2) computer software. Analysis involved line-by-line scrutiny of the transcript and assignment of keywords to text fragments. For each interview, categories and themes were identified; these categories and themes were subsequently tested and refined in a cyclic analytical process, moving backward and forward between the interviews. This type of analysis resembles the open coding and axial coding phases of grounded theory (Strauss, 1987). This approach was chosen for its ability to provide systematic inductive guidelines for collecting and analyzing data. To capture a more complete and contextualized picture of the data, we performed investigators' triangulation by having a co-investigator code two interviews independently. There was minor disagreement, which was resolved by discussion. Furthermore, we improved the credibility and transferability of the data by member checking. The results were presented to a group of the students who were asked to give feedback. A similar procedure was used with the interviewed teachers. KSAUHS ethics approval was obtained prior to conducting the research.

\section{Results}

We invited 56 from a total of 61 clinical year students to participate, and 28 of them agreed to do so. Each student took part in one of seven focus group interviews, with four students per group. The students' mean age was 26.74 years, and their mean graduation GPA was 3.96/5. These characteristics are similar to the mean age (26.77 years) and the mean GPA at graduation (3.89/5) of the non-participating students. Twelve teachers were approached, and they all agreed to take part in the study. 


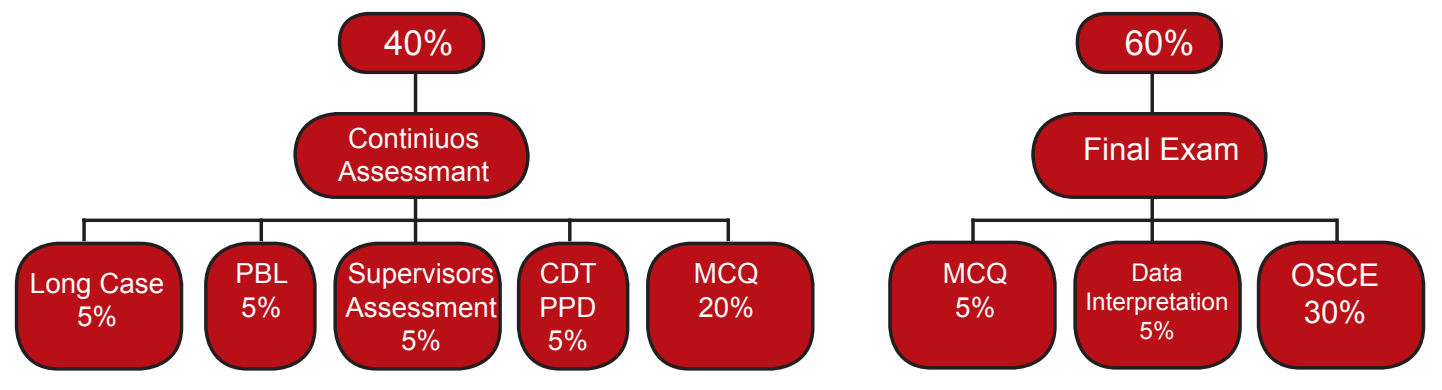

Figure 1: Flow chart of the Assessment Program in Years 3 and 4 at KSAU-HS, COM MCQ: multiple choice questions. PBL: problem-based learning. CDT: community doctor theme. PPD: personal professional development. OSCE: objective structured clinical examination.

The first analysis of the data generated four themes with its related codes (table 1). We noticed diversity in the level of abstraction and aggregation of codes within themes and between students and teachers. Twelve additional individual semi-structured student interviews were performed for a better understanding of the obtained themes. In order to illustrate how our concept of the effect of assessment on student learning is grounded in the data, we present quotes from the transcripts.

\section{Students' and teachers' perceptions of the assessment program; Summative versus formative assessment}

A majority of the students preferred summative exams as a discriminating factor between them, but they did not agree upon its effect on their future performance: "I would prefer summative assessment because I will have evidence to compare myself with others," and "I don't think it will reflects whether the students are good or bad." Some of the students were doubtful of the role of summative assessment in graduating better practicing doctors: "Student who had a full grade might not be able to deal with patients." On the other hand, a few students were not able to decide the 
superiority of summative or formative assessment role in their learning: "It's confusing whether to choose summative or formative assessment,; each one has advantages and disadvantages. "Several students said that summative assessment caused anxiety: "one hour of exam will determine your future; it is stressful." This stressful situation makes some students resort to sporadic, patchy reading through hunting the information they think is important or might come in their exam. Some started even to neglect thinking about the exam mark and focused only on passing it aiming to avoid this stressful feeling: "we have to read for the exam, we have to read for the clinical attachments; how can I get time in between, I am always stressed, very stressed." And "I don't care about the marks. If I care that much I'll be anxious and commit more mistakes..". Summative assessment was also considered by some students as unfair, it didn't reflect the effort they put and the activities they do across the block. Therefore some students were regretting the time they spent in preparing and performing these exams: "The exam is unfair... I feel I'm wasting my time for nothing. "In reaction to these complex opinions, some students became marks hunters practicing several study strategies aiming for passing the exam or scoring high marks. Of these strategies was holistic reading or on the contrary strategic, selective reading: "I will make sure that I could retain at least the minimum requirement," and "I read the important subjects like life threatening and emergency situations." Some students studied based on their own selected objectives, preferences, or type of exam. They used their own opinion, exam experiences, feelings and speculation in creating their hidden curriculum: "to pass the exam... I really need to remember some numbers, some percentages and things that can come in MCQ questions" and "I know some things about him or her that would make the examination easy for both of us.".

A majority of the students clearly preferred formative assessment due to its attached feedback. This feedback helped them to identify their learning objectives and improve their study strategies. The students' views contradicted the opinions expressed by 
some of the teachers, who stated that summative assessment was an essential step in students' assessment, leading to fair assessment. Teachers did not deny the importance of formative assessment. However they thought that it was time consuming.

Table 1: Themes within the coding system for both student and teacher interviews

\section{Theme}

1 Summative assessment

2 Formative assessment
Description

Codes on effect of summative assessment on students' learning

Codes on effect of formative assessment on students' learning
Codes

provoking anxiety; stressful; sporadic reading; has no role; a waste of time; unfair; summative is mandatory and more summative fair

Improve students' learning; affected by culture; time consuming; should not be overdone; fair; learning strategy improvement and problem identification

Should be given more weight; fair students' learning

Codes on the effect of objective directed assessment and constructive alignment on students' learning

Heavy; fair; content coverage; blueprinting; assessment weight

Formative affected by culture; changing culture; educational culture
Codes on the role of culture in students' learning and teachers' assessment 
If formative assessment is further increased, they may not be able to provide and maintain its performance; hence, it would be a waste of time. Interestingly, the expression "waste of time" was used by both students and teachers but with reference to different types of assessment. The teachers took into consideration the effect of the college's education culture on the implementation of formative assessment. "In our education culture, we focus on the exam and how much we achieve on the exam.". Both students and their teachers are mainly graduates of traditional curriculum where formative assessment was not part of their assessment methods. They thought that gradual adaptation to formative assessment and orientation of the learners and teachers to the process and to the goal of the process are important. Adequate time allowance recognized as essential prior to heavy formative assessment implementation.

\section{Continuous attachment-related assessment}

Assessments during clinical attachments (continuous assessment) were the composite of students' portfolios and were preferred by the students. It helped them improving their knowledge, and increasing self-awareness:" Continuous assessment actually encourages the student to read more and to keep up to the maximum level that is required from him.". The students felt that the weight placed on continuous assessment is very small. They emphasized that it should be sufficient enough to stimulate their clinical learning. Most of the teachers agreed with the students on the importance of clinical attachment assessment leading to better training: “...More weight for the Continuous Assessment, students will give more attention to the clinical attachment."

\section{Objectives}

An assessment program that reflects the curriculum's objectives was considered to be fair and reliable. It will enable them to correctly direct their reading based on what is planned for them in the curriculum: "I follow the objectives when I think about the exam.". Therefore students think that an assessment program should not be standardized 
across the blocks and should be individualized based on each block objectives. What was more important to the students is that their supervisors should be familiar with the curriculum objectives. The teachers noted various problems in relation to the objectives, indicating that these objectives should be simple and easily implemented. It should not be broad and the depth of knowledge and skills required should be specified. In their view, fair assessment was characterized by a strong alignment with curriculum objectives. Teachers stressed the importance of 'blueprinting' and 'assessment weight' in designing fair assessments: "A final exam should reflect all the curriculum components.".

\section{Discussion}

In this research, KSAU-HS, COM was taken as an example of a college that implement assessment program characterized by being mainly summative. The performed work was exploratory rather than definitive: its main value is to guide development of further enquiries and understanding. We kept in mind that even the best designed interventions will not always result in better learning for all students but for sure it will improve it at least for some.

In this study, the participating students said that they adapted their study strategies to task demands. The type of assessment and the weight accorded to it were significant factors that affected their approach to learning. This result is in agreement with other research, where assessment type (Pressley, Yokoi, Van Meter, Van Etten, and Freebern, 1997) and weight (Broekkamp Hein, Bernadette HAM, and Hout-Wolters, 2007) were identified as factors that influence students' approaches to learning.

The teachers in this study see more advantages of summative assessment than the students, with the latter group experiencing summative assessment as stressful, anxiety provoking, and inducing sporadic and superficial reading. In contrast to the teachers, 
the students express a preference for formative assessment with feedback. Formative assessment known to produce greater increase in students' achievement than class size reduction or increase in teachers' content knowledge (Dylan, and Thompson, 2008). In fact even summative tests can provide ways of eliciting evidence of student achievement. If used appropriately, can prompt feedback that moves learning forward (Black, and Dylan, 2009). However using grades alone for feedback is found to be the poorest type of feedback (Shute, 2008).

Formative assessment used in the presented research is considered as medium cycle formative assessment (every 1-4 weeks). The existing research base shows that shortand medium-cycle formative assessments improve student achievement (Popham, 2006). Having the students recognized the importance of this method of assessment despite its limited portion in the assessment program may be considered an extra evidence of its effectiveness.

The literature provides support mostly for the students' preferences, with a meta-analysis reporting that feedback produces the most powerful single effect on achievement (Hattie, 1978). The information processing needed for deep learning may be hampered when students do not spontaneously engage in cognitive activities that foster such learning (Sandberg, and Barnard, 1997). Research supports the use of learning materials and teaching methods that encourage students to employ deeper learning strategies whenever possible (Ross, and Tuovinen, 2001). To achieve this goal, the infrastructure needed to implement formative exams with appropriate accompanying feedback should be assessed. Formative feedback time, orientation of the learner to the process and to the goal of the process (Bienstock, Katz, Cox, Hueppchen, Erickson, and Puscheck, 2007) are essential. Ruston (Rushton, 2005) has discussed the presence of cultural difficulties to implement formative assessment and feedback. He questioned if the paradigm shift in assessment culture has occurred, as the majority of the existing 
literature is centered on summative assessment. The decision to accept and use formative feedback is influenced by several external and internal factors includes self perceptions, emotion, reflection and professional culture (Sargeant, Mann, Sinclair, Van der Vleuten, and Metsemakers, 2008). Through using historical data, it was clearly demonstrated how student perceptions have changed over time as a result of internal and external influences (Harvey, Moon, and Plimmer, 1997).

The students' remarks that summative assessment provokes anxiety and stress give pause for thought. The primary sources of stress have been repeatedly found to be examination and grades (Kumar, Dagli, Mathur, Jain, Prabu, and Kulkarni, 2009), therefore care should be directed to students' perceived feelings. The problem is not solely related to the introduction of summative versus formative exams; it is wider than this view. Balancing formative and summative assessments is part of the work needed to reduce student anxiety. To what extent formative assessment should be used at the expense of summative assessment is an area that needs further investigation. The studied group was in their clinical years, where PBL sessions were parallel to their work-based learning. The cognitive and emotional effects of small group learning in PBL are not clear enough in the literature (Dolmans, and Schmidt, 2006).

Anxiety in general affects the performance of any student. Joels (Joels, Pu, Wiegert, Oitzl, and Krugers, 2006) reported that stress within the context of a learning experience induces focused attention and improves remembering relevant information. Therefore, eliminating educational stress appears to be an impossible, unnecessary goal, whereas reducing unnecessary stress and improving the work environment should be targeted. Both the students and teachers preferred continuous attachment-related assessment. The main benefits of port folio use were improvements in students' knowledge and understanding, increased self-awareness, engagement in reflection, and improved student-teacher relationships (Buckley, Coleman, Davison, Khan, Zamora, Malick, 
Morley, Pollard, Ashcroft, Popovic, and Sayers, 2009). However, students' and teachers' time commitment required for portfolio completion and evaluation may be a major drawback even if a portfolio was required as part of students' assessment. The assessors should be well prepared, 'trained' as assessors, and perceived to be fair, competent, skilful, and knowledgeable (Neary, 2000). Therefore, adding the portfolio to the assessment program without appropriate infrastructure of faculty training and time allowance may contribute negatively to student anxiety and the educational culture.

The first priority in designing assessment program is to serve the purpose of promoting students' learning without forgetting the purpose of accountability, ranking and certifying competence. Assessment program that cover all the knowledge and competencies required based on Miller pyramids (Miller, 1990) is needed. While methods like MCQ, essay and oral exam can cover the knows and knows how levels. There is a need to implement types of work based assessment such as mini clinical examination (mini CEX), directed observation of practical skills (DOPS), long and short cases and others to test the students capabilities and competencies in both vivo and vitro. These should be accompanied by direct observation and feedback to allow improvement and guarantee competency. Overall, a careful balance between formative, summative, and continuous assessment (portfolio) needs to be attained. To decide what appropriate balance is, further researches are needed.

Students and teachers agree that an assessment program should be aligned with the curriculum objectives. Learning models that are guided by curriculum objectives are effective educational tools that help students achieve a broad and direct exposure to core educational concepts (Rapp, Gong, Reynolds, Lucioni, and Zagaja, 2007). Such a model will prevent the establishment of hidden curriculum, feelings of unfairness, and stress whenever an exam is conducted. Hence, it may stimulate a deep approach to learning. 
Some weaknesses of this study should be mentioned. The population consisted entirely of male students. We are not aware of previous comparisons between male and female students regarding their perceptions of exams, but a gender effect cannot be excluded. This study has discussed students' perceptions of their ability to learn. Further work on the effect of assessment on student learning is needed. Finally, the focus on one medical college maybe considered as narrow. However, we believe that the research impact is broad. The research has evaluated the assessment program taking into consideration the instructional design, curriculum used while reflecting on the other published research on students' assessment.

\section{Conclusion}

The results of this study confirm that assessment affects students' perceptions of learning and how they learn. These effects are not uniformly positive. According to the students in this study, the predominantly summative assessment program offers little inducement to engage in deep learning. They express a clear preference for formative and continuous assessment, which may foster a deeper approach to learning. It is important to be aware of the differing views held by students and teachers concerning the same educational assessment program. Faculty improvement programs and faculty time are important infrastructures for formative assessment implementation. Finally, efforts to achieve more clinically relevant assessment with adequate balance between the various types of assessment are required. Research is needed to decide this balance.

\section{References}

BAETEN, M., DOCHY, F. \& STRUYVEN, K. (2008). Students' approaches to learning and assessment preferences in a portfolio-based learning environment. Instructional Science 36, 359-374.

BIENSTOCK, J. L., KATZ, N. T., COX, S. M., HUEPPCHEN, N., ERICKSON, S. \& PUSCHECK, E. E. (2007). To the point: medical education reviews--providing feedback. Am J Obstet Gynecol 196, 508-13. BIGGS, J. (2003). Aligning teaching and assessing to course objectives, University of Averio. BLACK, P. \& DYLAN, W. (2009). Developing the theory of formative assessment. Educ Asse Eval Acc $21,5-31$. 
BROEKKAMP HEIN, BERNADETTE HAM, \& HOUT-WOLTERS, V. (2007). Students' Adaptation of Study Strategies When Preparing for Classroom Tests. Educ Psychol Rev 19, 401-428.

BUCKLEY, S., COLEMAN, J., DAVISON, I., KHAN, K. S., ZAMORA, J., MALICK, S., MORLEY, D., POLLARD, D., ASHCROFT, T., POPOVIC, C. \& SAYERS, J. (2009). The educational effects of portfolios on undergraduate student learning: a Best Evidence Medical Education (BEME) systematic review. BEME Guide No. 11. Med Teach 31, 282-98.

DOLMANS, D. H. \& SCHMIDT, H. G. (2006). What do we know about cognitive and motivational effects of small group tutorials in problem-based learning? Adv Health Sci Educ Theory Pract 11, 32136.

DYLAN, W. \& THOMPSON, M. (2008). Integrating assessment with instruction: What will it take to make it work? In Future of assessment: Shaping teaching and learning. Lawrence Erlbaum Associates, New York.

GIJBELS, D., SEGERS, M. \& STRUYF, E. (2008). Constructivist learning environments and the (im)possibility to change students' perceptions of assessment demands and approaches to learning. Instructional Science 36, 431-443.

HARVEY, L., MOON, S. \& PLIMMER, L. (1997). The Student satisfaction manual, Buckingham.

HATTIE, J. A. (1978). Identifying the salient factors of a model of student learning: a synthesis of metaanalyses. International Journal of Educational Research 11, 187-212.

JOELS, M., PU, Z., WIEGERT, O., OITZL, M. S. \& KRUGERS, H. J. (2006). Learning under stress: how does it work? Trends Cogn Sci 10, 152-8.

KUMAR, S., DAGLI, R. J., MATHUR, A., JAIN, M., PRABU, D. \& KULKARNI, S. (2009). Perceived sources of stress amongst Indian dental students. Eur J Dent Educ 13, 39-45.

LARSEN, D., BUTLER, A. \& ROEDIGER, H. (2008). Test enhanced learning in medical education. Med Educ 42, 959-966.

MATTICK, K. \& KNIGHT, L. (2007). High-quality learning: harder to achieve than we think? Med Educ 41, 638-44.

MCDANIEL, M., ROEDIGER, H. \& MCDERMOTT, K. (2007). Generalizing test-enhanced learning from the laboratory to the classroom. Psychonomic Bulletin \& Review 14, 200-206.

MESSICK, S. (1995). The interplay of evidence and consequences in the validation of performance assessments. Educational Research 13-23.

MILLER, G. E. (1990). The assessment of clinical skills/competence/performance. Academic Medicine 65, 63-67.

NEARY, M. (2000). Supporting students' learning and professional development through the process of continuous assessment and mentorship. Nurse Educ Today 20, 463-74.

NORTON, L. (2004). Using assessment criteria as learning criteria: a case study in psychology. Assessment and Evaluation in higher education 29, 687-702.

POPHAM, W. (2006). Phony Formative Assessment: Buyer Beware!. Educational Leadership 64, 8687.

PRESSLEY, M., YOKOI, L., VAN METER, P., VAN ETTEN, S. \& FREEBERN, G. (1997). Some of the reasons why preparing for exams is so hard: What can be done to make it easier? . Educational Psychology Review 9, 1-38.

RAPP, D. E., GONG, E. M., REYNOLDS, W. S., LUCIONI, A. \& ZAGAJA, G. P. (2007). Assessment 
of the core learning objectives curriculum for the urology clerkship. $J$ Urol 178, 2114-8.

REID, W. A., DUVALL, E. \& EVANS, P. (2007). Relationship between assessment results and approaches to learning and studying in Year Two medical students. Med Educ 41, 754-62.

ROSS, G. C. \& TUOVINEN, J. E. (2001). Deep versus surface learning with multimedia in nursing education development and evaluation of WoundCare. Comput Nurs 19, 213-23.

RUSHTON, A. (2005). Formative assessment: a key to deep learning? Medical Teacher 27, 509-513. SANDBERG, J. \& BARNARD, Y. (1997). Deep learning is difficult. Instructional Science 25, 15-36. SARGEANT, J., MANN, K., SINCLAIR, D., VAN DER VLEUTEN, C. \& METSEMAKERS, J. (2008). Understanding the influence of emotions and reflection upon multi-source feedback acceptance and use. Adv Health Sci Educ Theory Pract 13, 275-88.

SHUTE, J. V. (2008). Focus on Formative Feedback. Review of Educational Research 78, 153-189. STRAUSS, A. L. (1987). Qualitative Analysis for Social Scientists. Cambridge University Press, Cambridge. 
Chapter 4

\section{Students' perceptions of the impact of assessment on approaches to learning: A comparison between two medical schools with similar curricula}

A full text version was published as:

Kadri, H. M. A., Al-Moamary, M. S., Magzoub, M. E., Elzubeir, M., Roberts, C., and van der Vleuten, C. P. (2011). Students' perceptions of the impact of assessment on approaches to learning: a comparison between two medical schools with similar curricula. Int J Med Educ. 2, 44-52. 



\section{Abstract}

Objectives: The aim of the study was to investigate students' perceptions of assessment and the resulting learning styles.

Methods: Qualitative semi-structured interviews were conducted with 14 students and 8 clinical supervisors from Sydney Medical School and 12 students and 13 clinical supervisors from King Saud bin Abdulaziz University. Both institutions have similar curricula but a different assessment approach. The interviews were transcribed and analyzed using thematic analysis. Interview transcripts were stored and analyzed using ATLAS.ti.

Results: Three themes emerged from analyses of the interviews: the function of assessment, learning outcomes and, finally, authentic assessment in the clinical environment. A model is presented to show the relationship between contextual and different personal factors and students' perceptions of the impact of assessment on learning styles.

Conclusions: Cultural differences and emotions can affect students' perceptions of assessment and learning styles. A combination of formative and summative assessment based on learning objectives is required. This combination should take into consideration students' cultural background, values and the implemented education system. This balance should be sufficient to motivate students in order to maintain their focus and attention, and reduce the potential negative impacts of a hidden curriculum. The experience of authentic assessment was a powerful motivator for students' approaches to learning. 
Impact of assessment on approaches to learning

\section{Introduction}

It is well known that assessment is one of the most important factors affecting students' approaches to learning (Crooks and Mahalski, 1985, Ramsden, 1992, Scouller and Prosser, 1994, Thomas and Bain, 1984). Although many researchers emphasize this relationship,(Thomas and Bain, 1984, Newble and Jaeger, 1983, Al Kadri et al., 2009, Biggs, 1993, Biggs, 1999) it continues to be poorly understood particularly with regard to the following aspects: 1) the persistent incongruence between curricular and assessment objectives; 2) the purpose of assessment methods (formative/summative); and 3) the effect of personal influences, such as students' expectations for specific courses, academic discipline, prior education, age and gender, and cultural influences (Cooksey et al., 2007). Crooks et al. (Crooks, 1998) warned against the possible incongruence between academic objectives as intended by the curriculum and the objectives defined through the assessment process. Synchronization between these two types of objectives is called constructive alignment. When constructive alignment is achieved, it is assumed to be conducive to learning. Biggs (Biggs, 1979) and Ramsden (Ramsden, 1974, Ramsden, 1992) have described the interactive relationship among student factors, teaching context, the on-going approaches to a particular task and student learning outcomes. Through teaching and learning with consideration to this interactive relationship, learners will usually learn what should be learned. Therefore, one of the consequences of curriculum misalignment is that repeated discrepancies between what students perceive that they need to know for assessment purposes and the stated course objectives can potentially lead to a local culture, whereby a hidden curriculum 13 is created. Hafferty (Hafferty, 1998) defined the hidden curriculum as "a set of influences that function at the level of organizational structure and culture." Its nature depends on the students' own interests, supervisors' interests, and even students' personal speculations about what might be in their summative assessments (Al Kadri et al., 2009). 
In this study, the term "summative assessment" refers to an assessment performed to assign students a course grade, whereas the term "formative assessment" refers to an ungraded assessment that carries meaningful information as an educational tool to aid students' learning. Existing research on the effects of summative and formative assessment on students' learning strategies is unequivocal. Whilst some researchers claim that formative assessment is more effective than summative assessment in producing deep learning strategies, (Al Kadri et al., 2009, Rushton, 2005) others disagree (Torrance and Pryor, 1998, Hattie, 1978). Researchers have concluded that feedback and formative assessment produce the most powerful effect on student achievement (Crooks and Mahalski, 1985).

Formative assessment appears to play a larger role in increasing student achievement than does a reduction in class size or an increase in teachers' content knowledge (Dylan and Thompson, 2008). On the other hand, summative assessment is a proven way of eliciting evidence of student achievement and discriminating between students of differing abilities (Al Kadri et al., 2011, Black and Dylan, 2009). therefore similar to formative assessment, summative assessment may prompt feedback from faculty that promotes students' learning.

The third area of research where there is a lack of clarity is the effect of personal influences on students' approaches to learning. Students enter a course or a program with specific intentions about the study strategies that they are likely to employ (Biggs, 1993). These strategies are mediated by differing personal and contextual influences and the different ways by which students perceive assessment and its demands (Biggs, 1993, Al Kadri et al., 2009). Vermunt (Vermunt, 2005) tried to clarify the relationship between the way students learn and personal and contextual variables. He found that educational contexts like the way the learning environment is structured and personal factors; such as academic discipline, prior education, age and gender, had an effect on 
Impact of assessment on approaches to learning

students' learning patterns. For example, older or more experienced students showed greater ability to differentiate between various learning strategies than younger or less experienced students (Vermunt and Vermetten, 2004).

Furthermore, culture is a personal factor that encompasses students' beliefs, behaviors, attitudes, and practices that are learned, shared and passed on (Tervalon, 2003). Students' sense of a "cultural identity" (Dogra, 2001) is derived from a complex mixture of cultural, gender, social, economic, religious, and political affiliations. Little is known about how these different cultural factors might influence the way students perceive their assessment and affect their study strategies. Due to this inconsistency in our understanding of the effects of different personal and contextual factors on students' perceptions of the implemented assessment and their approaches to learning, further research is needed.

We were in a unique position to address our research aim by comparing students' perceptions of assessment and the students' resultant learning strategies in two programs in two different countries, with differing cultural values. At the time of the study, the King Saud Bin Abdulaziz University for Health Sciences, College of Medicine (KSAUHS, COM), Riyadh, Saudi Arabia had implemented a four-year graduate entry ProblemBased Learning (PBL) curriculum derived from that of Sydney Medical School (SMS). However, both had different assessment processes implemented during the third and fourth year of the curriculum (clinical years).

The assessment process during the clinical years in SMS was characterized by different requirements for each clinical block. During these blocks, students rotate in different clinical attachments where at the end of each they must complete a formative selfassessment form. This self assessment was done in parallel with supervisors' formative assessment and feedback. Students were also required to complete a variety of summative 
assessments, formative clinical exercises and online assessments that were differed from block to block. A multiple-choice question (MCQ) barrier exam was conducted by the end of the third year, which the students had to pass in order to progress to the final year. Based on their performance in their barrier exam, students were provided with summative feedback indicating their grades in each discipline and their standing in relation to their peers. Finally, a summative long case must be completed during the last year of the curriculum.

The assessment program for the third and fourth year of the curriculum at KSAUHS, COM was block-based and was similar from block to block. Students must pass all clinical blocks prior to their graduation. In each clinical block, students rotate in different clinical attachments where at the end of each they must complete formative self assessment form paralleled with supervisors' formative assessment and feedback. Moreover, in each block, students' assessment was divided into two main parts. The continuous assessment of students' performance accounted for $40 \%$ of the final grade, and the final block examination accounted for the other $60 \%$. In summary, the third and fourth years of the curriculum assessment program of KSAU-HS, COM in comparison to SMS assessment program was characterized by 1) uniformity from block to block,

2) less formative assessment, 3 ) more frequent structured summative assessments and 4) no barrier exams. The research aim of this study was to provide a theoretical insight into the interaction of different personal and contextual factors on students perception of the faculty implemented assessment in a clinical context and to understand their impact on students' resultant approaches to learning.

\section{Methods}

\section{Study Setting}

In both study settings, KSAU-HS, COM and SMS, third and fourth-year students rotate 
Impact of assessment on approaches to learning

through different clinical blocks, including medicine and surgery and specialist blocks. In each clinical block, students will join different clinical attachments supervised by clinical supervisors. This stage of the training program utilizes case based learning to augment direct patient contact.

\section{Study Design}

A qualitative approach using thematic analysis (Attride-Stirling, 2001) was used to generate a rich understanding of the full range of opinions and experiences of students when they are exposed to the implemented assessment. Our assumption was that students of different cultural background were influenced in their approach to learning by different personal and contextual factors. In interpreting our data we used a theoretical framework based on the work of Biggs (Biggs, 1979) and Ramsden (Ramsden, 1992, Ramsden, 1974) describing the interactive relationship among student factors, teaching context, the on-going approaches to a particular task and student learning outcomes.

\section{Study Population}

The study participants were students who were in the last two years of the curriculum. This convenience sampling was undertaken to gain students' common experiences and perceptions of the various methods of assessment implemented during this phase of the curriculum. In order to provide a richer insight into the contextual factors associated with working in a clinical placement, we also interviewed students' clinical supervisors. Accordingly, our data set incorporated the perspective of the experienced clinicians in charge of implementing the formal process of teaching and assessment.

At the time of the research conduction, KSAU-HS, COM was accepting only male students into the Medical program. All eligible students (61 students) and clinical supervisors (fifty six supervisors) were invited to participate in the study through an e-mail announcement and direct contact. Twenty eight students and all supervisors 
agreed to participate in the semi-structured individual interviews. We interviewed twelve students and thirteen supervisors, after which our analysis revealed data saturation.

In SMS, all third- and fourth-year students and their supervisors were invited to participate in the research through e-mail announcements. Those who accepted were called for interviews (fourteen students; eight males and six females, and eight supervisors) were interviewed after which our analysis revealed data saturation. The first author and a research assistant conducted interviews. At the time of the interviews, the first author was not involved in the academic experiences of the students participating in the study.

\section{Data Collection}

Semi-structured individual interviews and open-ended questions were conducted with students and supervisors. We explored students' experience of the curriculum, its learning objectives alignment with students learning activities and the given assessment. Furthermore, we explored students' experiences of feedback and the different assessment implemented with a particular focus on the ways in which students' practice had influenced their learning approaches. Each interview lasted from 30-45 minutes. Interviews were recorded on audiotape and transcribed verbatim.

\section{Analysis}

Interview data was examined in-depth aiming to obtain the emerging themes. Initial coding revealed a number of basic themes that were arranged to form organizing themes. Subsequently, organizing themes were iteratively discussed between authors and were renegotiated when differences existed. After further analysis, the organizing themes were condensed into the three global themes discussed in this paper (Attride-Stirling, 2001). The analysis of the transcriptions of the interviews, were managed using Atlas.ti (Version 5.2). 
Impact of assessment on approaches to learning

\section{Ethical Approval}

The approvals of the University of Sydney Human Research Ethics and KSAU-HS were obtained prior to conducting the research.

\section{Results}

A theoretical insight is presented that illustrates first, how students with different personal characteristics including their cultural backgrounds are influenced by contextual assessment related factors. Second, how this complex interaction affects their learning approaches. Our results are organized into three main themes: 1) students' personal perceptions of assessment function; 2) students' perceptions of learning outcomes; and 3 ) the student experience of authentic assessment in the clinical environment. We present these themes with illustrative quotes from students (S) and supervisors (T) from either SMS (U) or KSAU-HS (K).

\section{Students Personal Perceptions of Assessment Function}

\section{Summative Assessment}

Summative assessment was appreciated by all students due to its ability to provide students with a clear idea of their progress. It was perceived as a major factor in stimulating students to study more, exert more effort to pass an exam or get a higher mark; it can thus be considered as a stimulus to effort and achievement, as well as being a motivation strategy for study.

"If your exam counts and there will be a grade next to your name, you are going to be more serious when preparing for the exam" (SK).

The Saudi students particularly noted that this phenomenon related to their prior educational experience and the inherent importance given to summative assessment during their high school study and previous university degrees: 
“...we care about our marks, we study for the marks, ...we were raised like this. When we joined here...., problem based learning did not change us. We still focus on the marks....even our supervisors they focus mainly on our marks..." (SK).

Supervisors likewise perceived summative assessment as a stimulus for hard work, improved clinical performance, enhanced patients' safety and was a successful method in motivating students to increase their effort and improve their achievement. They believed that summative assessment could stimulate the development of better approaches to learning. Supervisors believed that students who pass their summative assessment would become better future doctors and therefore they recommended including this type of assessment in the curriculum assessment strategy:

"if students can't pass their summative assessment, that means they are not really suitable to be doctors" (TU).

Although summative exams lead to increased anxiety among the students, some of them were able to cope effectively. Our data suggests that coping methods could have been influenced by cultural background since students in each of the schools used different coping strategies. Stressful situations led KSAU-HS, COM students to resort to sporadic, patchy, superficial reading and to study the information they thought was important or might appear in the exam without going into the depth of the subject. These practices represent a superficial approach to learning:

"We have to study for the exam, we have to read for the clinical sessions; how can I get time in between? I am always stressed, very stressed" (SK).

Similarly, SMS students experienced stress when undergoing summative assessment, but they perceived this stress positively. It stimulated them to work hard, helped them develop deeper approaches to learning and enhanced their performance on exams: “.... the stress.... I like the stress...... stress helps. It motivates me. It is a little bit stressful but I think this is part of our learning" (SU). 
Impact of assessment on approaches to learning

We note that on top of the cultural differences, KSAU-HS, COM students' stress might have been related to the fact that they faced more frequent summative assessments. It may also be linked to their anxiety regarding future opportunities to enroll in postgraduate study, obtain a scholarship or even get a job, all of which could be determined by their accumulative assessment marks. On the other hand, SMS students were more confident of progressing as long as they passed all the assessment tasks.

Neither cultural differences nor differences in the implemented methods of assessment affected supervisors' perception of both students' anxiety and the purpose of summative exams. All supervisors considered this stress as a positive element that improves student learning, prepare them for patients care, future work challenges and helps students to practice effort and achievement motivation strategies while coping with this stress:

"So it is definitely the most stressful experience for students, but you know life is full of stress and if we just try to remove all stress, students will not be prepared to deal with real life stress." (TU)

Our data suggest that the learning approaches used by KSAU-HS, COM students to prepare for their summative assessment were generally more superficial and geared toward short-term results than were the approaches of the students at the SMS. These differences were a result of multiple personal and contextual factors, including different students' ability to cope with stress in the two study settings and the more frequent summative assessments faced by the KSAU-HS, COM students.

\section{Formative Assessment}

When the supervisors used formative assessment properly, it helped students to identify learning objectives and to improve their study strategies. This resulted in an improved ability of students to diagnose patients' diseases, apply theoretical knowledge to patient care, and plan appropriate management strategies. Therefore, formative assessments stimulated meaningful and multifaceted learning. However, students in SMS were more 
capable of accommodating negative feedback and perceiving it positively than were KSAU-HS, COM students. The SMS students were more enthusiastic about receiving and using direct verbal feedback:

"I like negative feedback, I think it is good. To me it just tells me to go and read more... you need to be reminded to take care" (SU).

It appears that cultural values and preferences may have contributed to the KSAU-HS, COM students' perception of negative feedback and their ability to accommodate it. They considered negative feedback as criticism carrying no constructive value: "negative feedback puts students under more stress. You work hard for long time and at the end your performance is assessed to be poor..." (SK).

Students and supervisors in both settings disagreed about the suitable frequency of formative assessment and feedback. The recommended frequency ranged from once every few months to twice every week:

"A formative exam may be once every two blocks, you know just to get people to know what the barrier exams are going to be like." (SU) and "at least once or twice a week and not less than that." (SU).

This wide range maybe attributed to the differing perceptions of the appropriate amount of time and effort necessary for completion and evaluation of assignments.

\section{Students Perception of Learning Outcomes}

Students considered assessment fair and a stimulus to deep approach to learning when it was tailored to curriculum objectives. When the formulated objectives were neither specific nor precise, students directed their studying towards selected objectives. They used their own opinions, exam experiences, feelings and speculations in creating their own curriculum. The tendency to create a hidden curriculum 13 was more prevalent among the SMS students, which may reflect a better understanding of the curriculum implementation and assessment process and greater academic independence. The 
Impact of assessment on approaches to learning

more formative emphasis of the SMS assessment program may have contributed to the students' behavior. On the contrary, the more summative emphasis of KSAU-HS assessment program made the students more hesitant to create their own study agenda or a hidden curriculum:

"I didn't find the objectives very useful. I'll never really study in that way. I am happier to just focus mainly on the clinical stuff....so it's more of personal preferences." (SU) and "Usually I start by looking into the objectives and I make sure that when I study I read these points specifically... I found that quite helpful...." (SK).

The presence of broad, imprecise objectives across the curriculum have resulted in variations in students' understanding of their assignments and therefore variations in their approaches to learning. Students who studied based on the curriculum objectives have opted to study as much as possible of the many topics covered in their objectives without focusing on their importance, relevance or linking it to patients' management. That lead to superficial approach to learning. Others have opted to create their own objectives based on preferences, senior advises or what they have observed as important information during their direct patients contact. Therefore, they studied based on these selected objectives and linked it to patients' care resulting in deep approach to learning for selected topics and superficial approach to learning for others. These approaches variations in response to broad imprecise objectives did not differ between the two studied groups of students with higher tendency to create a hidden curriculum between SMS students:

"...the problem I have found is that there is no guide to how much you need to know on that subject. It is too broad...you can read a textbook or you can read a very brief definition...it usually depends on the students themselves how much they decide to study "(SU) and "To read based on the objectives....that will take a lot of time. What I usually do, is to look into the information given to us... and just study this information and expand on the points that I need..." (SU). 
What made the hidden curriculum even more prevalent is students' perception of their learning outcome and its relation to their assessment. Students stressed on the role of personal values and preferences on their learning approaches. They thought that safe patients care and good practice are more motivating for quality studying compared with faculty assessment. This perception was not affected by the different cultural and contextual differences between the two studied groups:

"I have a certain expectation of myself; I would perform indeed as a safe practitioner, and to be a safe practitioner. I need to know my limits and I need to know when to ask for help. I do not think the assessments we have really reinforce these points" (SU). Direct patients contact and the authentic assessment in the work place were connected to a better learning approach, which directs us to our third identified theme.

\section{Student Perception of Authentic Assessment in the Workplace}

All students in both settings preferred and appreciated observed clinical assessment. They perceived it as reflecting their future performance and patient-care capabilities. Constructive and direct supervision helped students integrate clinical knowledge into practice, summarize patients' histories and solve patients' problems. When supervisors acted as role models, their methods of coaching and guiding students, experience and commitment to clinical teaching were crucial factors in enhancing the quality of learning and improving students' performance on exams.

Direct patient encounters improved students' performance and confidence in performing clinical examinations and enhanced student learning. It helped students in directing their efforts towards solving their patients' problems and encouraged clinical work. However, in both study settings, there were variations in students' responses to their clinical experiences. It appeared that, in addition to direct patient encounters, general and personal factors are needed to enhance students' motivation. Moreover, supervisors' investment of time, interest and teaching skills were important enhancements. 
Impact of assessment on approaches to learning

Supervisors felt that an appropriate balance between clinical tasks and teaching motivated them to involve students in patient management and to consider them as part of the managing team. When the clinical supervisors had more teaching time and experience, they were more capable of stimulating students' interest in clinical training and fostering favorable learning conditions:

"The most important trigger for students to learn is their new experience in the hospital. The resulting excitement...stimulates them to do more reading and preparation for assessment" (TK); "I'm not sure that all students have the same preferences. There is a student who is supposed to be my partner in this rotation; he has not been seen for the last two weeks. Obviously that person has not turned out for anything at all." (SU).

\section{Discussion}

Our study has provided an insight into the interactive relationship between students' personal values, cultural factors, teaching context, their perceptions of assessment tasks and the students' resultant learning approaches as they progressed through their clinical placements. Important factors affecting students' perceptions of their assessment included the type of assessment used (summative or formative), their perception of curriculum objectives and learning outcome, and the presence and nature of authentic clinical assessment with the involvement of clinical supervisors. Variations in students' study strategies were influenced by; firstly different contextual processes of curriculum (including assessment) implementation; and secondly, by students' cultural backgrounds and personal values and preferences. These different perceptions have resulted in differences in students' learning approaches (figure 1).

The cultural differences between the two studied groups appears to have influenced their perception of learning and their approach to their studying (Tervalon, 2003). This resonates with previous research on cross-cultural learning styles and learning behaviors which has recommended that students' approaches to studying must be interpreted in the educational, institutional and cultural contexts within which the study 
occurs (Sadler-Smith and Tsang, 1998). This partly reflects prior educational experience and the prevailing educational systems in differing cultures. For example, in one study, Asian students tend to have difficulty adjusting to an educational environment that is characterized by independent learning and less instructor supervise on and guidance (Watkins and Biggs, 1996). Saudi students similarly are thought to have had a teacheroriented, lecture-style learning environments (Shaw, Unpublished Doctoral Thesis) prior to medical school.

In this study, both studied groups acknowledged the importance of summative assessment as a motivator for hard work. However, in contrast to SMS students, the KSAU-HS, COM students perceived summative assessment as a stressful and anxietyprovoking experience that led them to engage in sporadic and superficial reading. Therefore, the summative system may have both positive and negative influences on students' learning. These influences depend upon students' perceptions. The behavioral symptoms displayed by the KSAU-HS, COM students during preparation for and completion of summative exams might be what is known as "test anxiety". Based upon the literature, about $10 \%$ of students suffer from test anxiety, which compromises their performance and emotional well-being (Schaefer et al., 2007, Latas et al., 2010). This problem is not specific to KSAU-HS, COM students. Severe test anxieties have been reported for medical trainees in many countries with different social and financial backgrounds, including the United States, Australia, China, England, Germany, India, Italy, the Netherlands, Pakistan and Turkey (Powell, 2004).

It was found that the primary source of test anxiety was exam grades (Kumar et al., 2009). Severe anxiety symptoms were reported to occur in anticipation of and/or during professional licensing examinations, particularly those that contained test questions in a multiple-choice format (Powell, 2004). Therefore, the summative characteristics of the Saudi college assessment program may have led students to experience adverse 
Impact of assessment on approaches to learning

cognitive and emotional effects, including impaired attention, problems with focusing and difficulties with the retrieval of stored knowledge (Braunstein-Bercovitz et al., 2001). These problems, in turn, may have adversely affected their study habits (Smith et al., 1990), leading to a more superficial approach to learning.

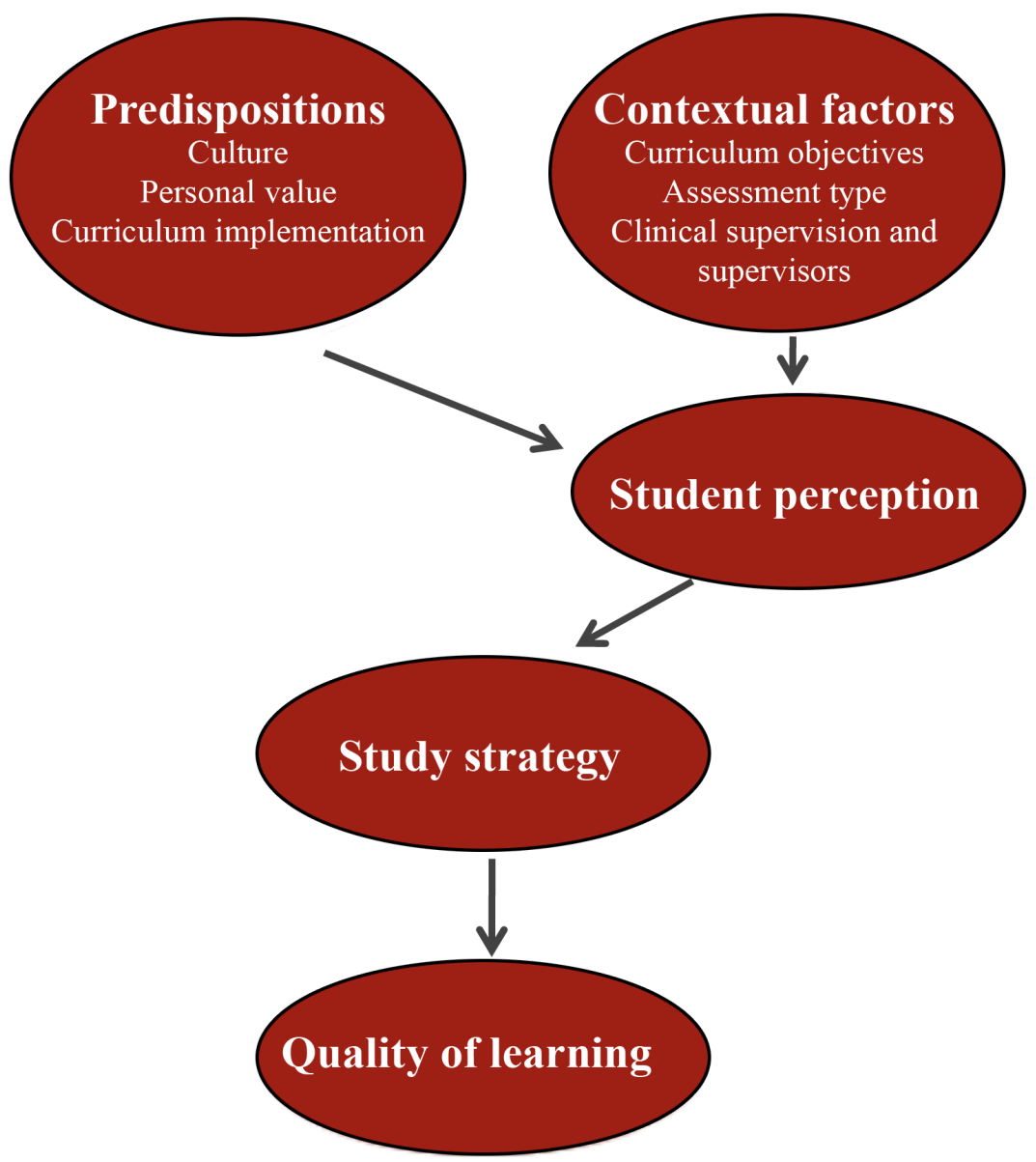

Figure 1: The effect of different contextual factors and various predispositions on students' perception of their assessment and learning 
The SMS students were subjected to less frequent summative assessment but to more formative assessment compared to KSAU-HS, COM students. It appeared that the balance between summative and formative assessment in the SMS program reduced the negative anxiety effects of summative assessment. Moreover, the possible influence of cultural factors in enabling SMS students to cope with this anxiety and its subsequent effect on their perception of the assessment process cannot be ruled out. The assessment program in SMS may have created just the right degree of stress, leading to focused attention, improved memory (Joels et al., 2006) and better overall results. Our data suggest that student perceive stress from summative exams as beneficial to a certain degree, beyond which it has negative effects. Therefore, negative stress resulting from an over focus on summative assessment should be balanced with adequate frequency of formative feedback.

Both groups of students and supervisors recognized the importance and positive impact of formative assessment on students' learning. Despite this agreement, it appears that the presence of cultural differences between the two groups influenced students' responses to negative feedback. KSAU-HS, COM students' self-perception, emotions and professional culture have affected their ability to accept and accommodate supervisors' negative feedback (Sargeant et al., 2008, Harvey et al., 1997).

Curriculum alignment of learning objectives, assessment and teaching and learning activities help students to achieve broad and direct exposure to core educational concepts (Rapp et al., 2007). However, many students were actually selecting their own study objectives, thereby creating a hidden curriculum (Hafferty, 1998). The prevalence of this phenomenon among SMS students may have been a result of the less structured and formative nature of assessment compared with KSAU-HS, COM assessment program. KSAU-HS, COM assessment program, with its frequent summative assessments and highly structured format, was more successful in stimulating curriculum objectivebased learning and in reducing the tendency to create a hidden curriculum. However, the 
Impact of assessment on approaches to learning

emphasis on structured summative assessment has led to more anxiety and a tendency towards a superficial and achievement motivation study strategies.

In fact, the health-education system in Saudi Arabia may have contributed to the development of such attitudes among KSAU-HS, COM students. A student's prospects for residency training in Saudi Arabia are greatly dependent on their accumulative assessment grades. Consequently, students develop a very competitive attitude with the goal of achieving high scores, and this attitude makes them less likely to gamble in selecting their study objectives.

There were similarities in students' perceptions of clinical assessment as opposed to written assessment in between the two studied groups. Students particularly appreciated work based assessment that was conducive to learning and held significant value for them. Work-based assessment was perceived by the students as leading to more skilled doctors and was a stimulant for better approaches to learning. When students begin clinical training, they encounter different supervised learning environments and different assessment programs. In these learning environments, supervisors' knowledge, skills, encouragement of a problem-solving approach, critical reflection on practice, supervision and assessment methods are perceived as important factors affecting students' study strategies (Marrow and Tatum, 1994).

The results of this study regarding the importance of available time for teaching and clinical assessment are also confirmed by other studies (Hayes, 2008, Wimmers et al., 2006). Variations between institutions and supervisors in the acceptance of responsibility for clinical teaching and time allocated for supervision have been reported. In this study, limited time and resources for clinical teaching were regarded as barriers to highquality teaching performance. This result was not affected by the various differences between the two groups. 


\section{Study Limitations}

One of the limitations of this study is related to the study population; at KSAU-HS, COM the student population is entirely male, whereas SMS has a mixed population of male and female students. A second limitation lies in the difficulty of comparing two very different groups. Additional cultural factors may have contributed to differences in study strategies, for example the differences between the multi-cultural society of Sydney and the ethnically homogenous Saudi student population at KSAU-HS, COM. Additional contextual factors could include the length of supervisors' teaching experience. Furthermore, despite the fact that similar curricula were implemented in both schools, we cannot deny the possibility of curricular differences.

\section{Conclusion}

Differences in assessment methods appear to lead to different perceptions and learning approaches; this might be mediated by some differences in cultural values. To maximize the educational impact of assessment programs and to avoid the possible negative effects of cultural barriers, a combination of formative and summative assessment is needed accompanied with precisely written curriculum objectives. There should be a balance between summative and formative assessment to stimulate stress, which helps students focus their attention, improve their performance and avoid the creation of a hidden curriculum. Such assessment programs should be tailored for each institution, taking into consideration not only assessment factors but also cultural values, preferences, health-education systems and job opportunities. Furthermore, students and supervisors should be prepared prior to the implementation of formative assessment through adequate orientation and faculty development programs. Such preparation will allow students to accommodate and benefit from negative feedback. Finally, there is a paucity of research in cross-cultural teaching and learning in a medical education context and we recommend further research that focuses on the cultural role on students' perception of their assessment and the resulting study strategies. 


\section{References}

AL KADRI, H. M., AL-MOAMARY, M. S., ElZUBAIR, M., MAGZOUB, M. E., ALMUTAIRI, A., ROBERTS, C. \& VAN DER VLEUTEN, C. (2011) Exploring factors affecting undergraduate medical students' study strategies in the clinical years: a qualitative study. Adv Health Sci Educ Theory Pract. DOI. 10.1007/s10459-010-9271-2.

AL KADRI, H. M., AL-MOAMARY, M. S. \& VAN DER VLEUTEN, C. (2009) Students' and teachers' perceptions of clinical assessment program: A qualitative study in a PBL curriculum. BMC Res Notes, $2,263$.

ATTRIDE-STIRLING, J. (2001) Thematic networks: an analytic tool for qualitative research. Qualitative Research, 1, 385-405.

BIGGS, J. (1979) Individual differences in study processes and the quality of learning outcomes. Higher Education, 9, 114-125.

BIGGS, J. (1993) What do inventories of students' learning processes really measure? A theoretical review and clarification. Br J Educ Psychol, 63, 3-19.

BIGGS, J. (1999) Teaching for Quality Learning at University, Buckingham, SRHE and Open University Press.

BLACK, P. \& DYLAN, W. (2009) Developing the theory of formative assessment. Educ Asse Eval Acc, 21, 5-31.

BRAUNSTEIN-BERCOVITZ, H., DIMENTMAN-ASHKENAZI, I. \& LUBOW, R. E. (2001) Stress affects the selection of relevant from irrelevant stimuli. Emotion, 1, 182-92.

COOKSEY, R. W., FREEBODY, P. \& WYATT-SMITH, C. (2007) Educational Research and Evaluation, 13, 401-434.

CROOKS, T. J. (1998) The impact of classroom evaluation practices on students. . Review of Educational Research, 58, 438-481.

CROOKS, T. J. \& MAHALSKI, P. A. (1985) Relationships among assessment practices, study methods, and grades obtained. Research and Development in Higher Education, 8, 234-40.

DOGRA, N. (2001) The development and evaluation of a programme to teach cultural diversity to medical undergraduate students. Med Educ, 35, 232-41.

DYLAN, W. \& THOMPSON, M. (2008) Integrating assessment with instruction: What will it take to make it work? Future of assessment: Shaping teaching and learning. New York, Lawrence Erlbaum Associates.

HAFFERTY, F. (1998) Beyond curricular reform: confronting medicine's hidden curriculum. Academic Medicine, 73, 403-407.

HARVEY, L., MOON, S. \& PLIMMER, L. (1997) The Student satisfaction manual. SRHE / Open University Press. Buckingham.

HATTIE, J. A. (1978) Identifying the salient factors of a model of student learning: a synthesis of metaanalyses. International Journal of Educational Research, 11, 187-212.

HAYES, R. (2008) Assessment in medical education: roles for clinical teachers. The Clinical Teacher, 5, 23-27. JOELS, M., PU, Z., WIEGERT, O., OITZL, M. S. \& KRUGERS, H. J. (2006) Learning under stress: how does it work? Trends Cogn Sci, 10, 152-8.

KUMAR, S., DAGLI, R. J., MATHUR, A., JAIN, M., PRABU, D. \& KULKARNI, S. (2009) Perceived 
sources of stress amongst Indian dental students. Eur J Dent Educ, 13, 39-45.

LATAS, M., PANTIC, M. \& OBRADOVIC, D. (2010) Medical students test anxiety. European Psychiatry, 25, 707.

MARROW, C. E. \& TATUM, S. (1994) Student supervision: myth or reality? J Adv Nurs, 19, 1247-55.

NEWBLE, D. \& JAEGER, K. (1983) The effect of assessment and examination on the learning of medical students. Med Educ, 17, 165-171.

POWELL, D. H. (2004) Behavioral treatment of debilitating test anxiety among medical students. J Clin Psychol, 60, 853-65.

RAMSDEN, E. L. (1974) Behavioral Science in Medical Education: A Learning Model. Journal of Med Educ, 49, 1182-83.

RAMSDEN, P. (1992) Learning to teach in higher education, London, Routeledge

RAPP, D. E., GONG, E. M., REYNOLDS, W. S., LUCIONI, A. \& ZAGAJA, G. P. (2007) Assessment of the core learning objectives curriculum for the urology clerkship. $J$ Urol, 178, 2114-8.

RUSHTON, A. (2005) Formative assessment: a key to deep learning? Med Teach, 27, 509-513.

SADLER-SMITH, E. \& TSANG, F. (1998) A comparative study of approaches to studying in Hong Kong and the United Kingdom. British Journal of Educational Psychology Review, 68, 81-93.

SARGEANT, J., MANN, K., SINCLAIR, D., VAN DER VLEUTEN, C. \& METSEMAKERS, J. (2008) Understanding the influence of emotions and reflection upon multi-source feedback acceptance and use. Adv Health Sci Educ Theory Pract, 13, 275-88.

SCHAEFER, A., MATTHESS, H., PFITZER, G. \& KOHLE, K. (2007) [Mental health and performance of medical students with high and low test anxiety]. Psychother Psychosom Med Psychol, 57, 289-97.

SCOULLER, K. \& PROSSER, M. (1994) Students' experiences in studying for multiple-choice question examinations. Studies in Higher Education, 19, 267-279.

SHAW, D. (Unpublished Doctoral Thesis) Bridging Differences: Saudi Arabian Students Reflect on Their Educational Experiences and Share Success Strategies Oregon State University.

SMITH, J., ARNKOFF, D. B. \& WRIGHT, T. L. (1990) Test anxiety and academic competence: A comparison of alternative models. Journal of Counseling Psychology, 37, 313-321.

TERVALON, M. (2003) Components of culture in health for medical students' education. Acad Med, 78, 570-6.

THOMAS, P. R. \& BAIN, J. D. (1984) Contextual dependence of learning approaches: the effects of assessment. Human Learning, 3:, 227-240.

TORRANCE, H. \& PRYOR, J. (1998) Investigating Formative Assessment: teaching, Learning and Assessment in the Classroom. Open University Press. Buckingham.

VERMUNT, J. D. (2005) Relations between student learning patterns and personal and contextual factors and academic performance. Higher Education, 49, 205-34.

VERMUNT, J. D. \& VERMETTEN, Y. J. (2004) Patterns in Student Learning: Relationships Between Learning Strategies, Conceptions of Learning, and Learning Orientations. Educational Psychology Review, 16, 359-384.

WATKINS, D. \& BIGGS, J. (1996) The Chinese Learner: Cultural, Psychological and Contextual Influences, Melbourne, CERC \& ACER.

WIMMERS, P. F., SCHMIDT, H. G. \& SPLINTER, T. A. W. (2006) Influence of clerkship experiences on clinical competence. Med Educ, 40, 450-458. 



\section{Chapter 5}

\section{Self-assessment and students' study strategies in a community of clinical practice: A qualitative study}

Provisionally accepted for publication as:

Al-Kadri, HM., Al-Moamary, MS., Al-Takroni, H., Roberts, C., and van der Vleuten, CPM. (2011). Self-assessment and students' study strategies in a community of clinical Practice: A qualitative study. Med Educ Online Journal. Under Processing. 



\section{Abstract}

Background: Self-assessment is recognized as a necessary skill for lifelong learning. It is widely reported to offer numerous advantages to the learner. We have evaluated in this research the impact of students and supervisors' self-assessment and feedback training on students' perceptions and practices of self-assessment. Moreover, we evaluated the effect of self-assessment process on students' study strategies within a community of clinical practice.

Methods: We conducted a qualitative phenomenological study from May 2008 to December 2009. We held Thirty-seven semi-structured individual interviews with three different cohorts of undergraduate medical students until we reached data saturation. The three cohorts were exposed to different contexts while experiencing their clinical years' assessment program. In the interviews, students' perceptions and interpretations of "self-assessment practice" and "supervisor-provided feedback" within different contexts and the resulting study strategies were explored.

Results: The analysis of interview data with three different cohorts of students yielded three major themes. These were strategic practice of self-assessment, self-assessment and study strategies and feedback and study strategies. It appears that self-assessment is not appropriate within a summative context, and its implementation requires cultural preparation. Despite education and orientation on the two major components of the selfassessment process, feedback was more effective in enhancing deeper study strategies.

Conclusion: This research suggests that the theoretical advantages linked to the selfassessment process are a result of its feedback component rather than the practice of self-assessment isolated from feedback. Further research exploring the effects of different contextual and personal factors on students' self-assessment is needed. 


\section{Introduction}

Self-assessment has been recognized as a necessary skill for lifelong learning (Dearnley and Meddings, 2007, Boud, 1995). It is widely reported to offer numerous advantages to the learner. The resulting dialogue between students and teachers and the skills acquired through self-assessment processes have been found to enhance student achievement, critical awareness, and reflection on learning (Black and Wiliam, 1998, Dearnley and Meddings, 2007). Therefore, self-assessment promotes a deeper approach to learning (Ramsden, 1992). However, research suggests that students' perceptions and the practice of self-assessment are unstable skills that can be affected by variables such as students' gender, degree of ability, length of practice, breadth of the studied area and understanding of the criteria necessary to judge and effectively interpret their own work (Boud, 1989). This instability leads to inconsistent effects of self-assessment practice on students' self-regulation and even on their self-direction as lifelong learners (Kirbya and Downsa, 2007). Moreover, this instability leads to inconsistencies in the strategies used by students when their self-assessment practice leads to an over or underestimation of their performance. It also results in poor agreement between students' self-assessment ratings and their supervisors' in course performance, particularly in student ratings as measured by the supervisor (Woolliscroft et al., 1993).

Self assessment is defined as "the involvement of students in identifying standards and/or criteria to apply to their work and making judgments about the extent to which they have met these criteria and standards" (Boud, 1995). In fact self assessment is identified to be a complex task; "it is a pedagogical process by which a student undertakes the task of looking outward to seek feedback and explicit information from external sources and uses these externally generated sources of assessment data to direct performance improvement" (Eva and Regehr, 2008). The process of self-assessment then requires two main skills: 1) skills on identifying self-ability in comparison to the required standards for a task and 2) skills on seeking and using constructive feedback 
(Boud, 2000, Dearnley and Meddings, 2007, Kruidering-Hall et al., 2009). Students' enrichment with these specific self-assessment skills leads to enhancement of their empowerment and reduction of the unilateral assessment power of the academic staff (Stefani, 1998, McMahon, 1999, Butcher and Stefani, 1995, Rainsbury and Hodges, 1998, Somervell, 1993, Boud, 1995). Therefore, it may increase students' interest, motivate them and shift the assessment process from an assessment of learning to an assessment for learning (Wood, 2009, Dearnley and Meddings, 2007).

In the clinical context, students' knowledge of their training requirements, their assessment and their supervision create a common ground for learning; guiding their study strategies and giving meaning to their actions and interactions. A community of students and supervisors in the clinical context creates the social fabric of students' learning, fostering surroundings and interrelations that can be called a "community of clinical practice" (Al Kadri et al., 2011). In the community of clinical practice, self-assessment is interpreted as a learning experience that can stimulate learning by identifying standards and providing suggestions for improvement (Colliver et al., 2005). Teachers in the community of clinical practice, make an invaluable contribution to students' learning (Dolmans et al., 2002, Stern et al., 2000) and face the difficult task of meeting two important objectives; meeting high performance standards for service and teaching and optimizing students' learning experiences by providing support, direction and constructive feedback (Kilminster and Jolly, 2000).

Most of the published research within clinical practice has quantitatively evaluated students' self-assessment scores and compared them to the scores given by their teachers. To our knowledge, there has been no reflection on the effect of self-assessment on study strategies subsequently practiced by the students. Furthermore, research on self-assessment within the medical education literature is lacking a specific theoretical framework in its analysis. Within the community of clinical practice, summative 
assessment has resulted in the enhancement of students' motivation to learn at the cost of superficial approach and achievement-motivation approach to learning (Vermunt, 1998, Vermunt, 1996). However, formative assessment with feedback has resulted in a tendency toward a deep approach to learning (Vermunt, 1996, Vermunt, 1998, Al Kadri et al., 2011). We are not aware of any research that has discussed the effect of self-assessment and the rule of effective training on self-assessment process on students' study strategies in a clinical setting. Therefore, we aimed in this paper to explore the relationship between students' perceptions and practices of self-assessment and their study strategies within a community of clinical practice. Moreover, through the conduction of this research, we aimed to assess the impact of student and supervisor self-assessment and feedback training on students' perceptions and practices of self-assessment.

\section{Methods}

We conducted a qualitative, phenomenological study to explore the values, cultural influences, orientation/education influences and strategies used by the students while practicing self-assessment in the community of clinical practice. To conduct this research, we structured our self-assessment practice and research to allow an in-depth exploration of different contexts that may affect students' practice of self-assessment. We implemented an integrated self-assessment by the students and a summative assessment by the supervisors, in which the students self-assess and then compare their assessment with their supervisors' summative evaluations "both students and supervisors assessments are performed on the same form parallel to the students' selfassessment". Finally, the students receive constructive feedback and a plan for their future learning from their supervisors. We performed semi-structured individual interviews to provide students with the freedom to express their perceptions and practice of the two main components of self-assessment process and the resulting study strategies. To provide a richer understanding of our research questions, we manipulated the contexts surrounding our research in particular the interaction between summative assessment 
and self-assessment and student and supervisor orientation on self-assessment and feedback. We observed students practicing the process of self-assessment in different contexts and sampled three successive cohorts of students.

\section{Study Setting}

The study was conducted at the College of Medicine (COM), King Saud bin Abdulaziz University for Health Sciences (KSAU-HS), Riyadh from May 2008 to December 2009. The college accepts only male students and is housed within King Abdulaziz Medical City, a 1000-bed tertiary care teaching hospital. The KSAU-HS, COM curriculum is a hybrid problem-based learning (PBL) curriculum. It is a four-year, integrated, graduateentry program consisting of a two-year pre-clinical phase and a two-year of clinical phase. The PBL extends into the clinical years along with direct patients encounters. The students practiced self-assessment during the clinical years of the curriculum only.

\section{Assessment Program}

The assessment program for the clinical years at KSAU-HS, COM is block-based. In each block, the student assessment is composed of two main parts. The first part is continuous assessment practiced throughout the block, which accounts for $40 \%$ of each block grade. This part includes students' clinical attachment evaluation, the midblock Multiple Choice Question (MCQ) exam, students' PBL sessions evaluation and finally, their personal and professional development and community doctor sessions evaluations. The Final examination accounts for the other $60 \%$ of the block grade that consist of the Objective Structured Clinical Examination (OSCE) and the MCQ.

\section{Self-Assessment Practice}

The implemented curriculum at KSAU-HS, COM during the clinical phase of students training (third and fourth year) is block based through the implementation of five major blocks. Internal Medicine and Surgical Blocks are conducted during the first year of the 
clinical phase of students learning. Obstetrics and Gynecology, Pediatrics and Family and Community Medicine blocks are conducted during the second year of the clinical phase of students learning. Each clinical block is composed of several clinical attachments during which students rotate in different specialties and sub-specialties and are attached to different clinical supervisors. For example in the Obstetrics and Gynecology block (9 weeks block), students will experience five different clinical attachments. Four weeks attachment in the general Obstetrics and Gynecology division in which each three to four students are attached to one clinical supervisor. To follow, students rotate in four other different Obstetrics and Gynecology clinical attachments, (one week each). These are Labor and Delivery, Perinatology, Neonatology and Reproductive Endocrinology attachments. The final week of each clinical block is usually left for the clinical and written final exams. In all the clinical attachments, supervisors are requested to implement curriculum objectives, guide their students, teach, assess and provide them with constructive feedback. At the end of each clinical attachment within each block, students work to arrange for a meeting with their supervisor. During this meeting, students should provide their supervisors with their self-assessment for that clinical attachment. This self-assessment includes student performance in the domains of knowledge, clinical skills, and communication as well as professional and ethical aspects. Following a discussion of the student's self-assessment, the supervisor provides the student with 1) a parallel summative assessment of the student's performance in the clinical attachment and 2) written and verbal feedback that aims to guide the student's future progress. The supervisors' clinical attachment assessment provides $5 \%$ of the total block grade.

Educational/orientation workshops (Implemented for the second and third cohorts of students)

We designed separate student and clinical supervisor half day orientation workshops aiming to assess the effect of students and faculties orientation on self-assessment on students' perception of this type of assessment. Students' workshops were implemented 
on the second and third cohorts of students only. The process was accompanied with a practical session on how to practice self-assessment and how to utilize supervisor feedback. The supervisors' workshops were started after the completion of the first cohort of students' interviews and were accompanied by a practical session on how to provide constructive feedback. We repeated these workshops for both students and supervisors until we ensured that all clinical supervisors who were eligible to participate in the research and all eligible students had attended the training activity. Time for questions and discussion and a contact person for further advice were provided.

Separating the summative assessment process from the self-assessment process (Implemented on the third cohort of students only)

To assess the summative impact on the students' perception and practice of selfassessment, we separated the summative assessment process from the self-assessment process for a third cohort of students who had attended the orientation program on selfassessment and feedback.

We modified the previously implemented integrated summative/self-assessment process by separating the two processes while maintaining identical assessment forms for the students and supervisors. Based on the implemented changes, at the end of each clinical attachment, students completed the self-assessment forms and submitted them independently to the block secretary. The student's supervisor completed the clinical attachment summative assessment without having seen the student's self-assessment. The supervisor, in turn, submitted the form to the block secretary. Subsequently, both the student and the supervisor met while both forms are available to them. The student then received supervisor feedback and an improvement plan. Therefore, while the first two cohorts of interviewed students performed their self-assessment parallel to their supervisors' summative assessments and on the same form, the third cohorts of students' self-assessments and their supervisors' summative assessments were performed independently on two different forms. 


\section{Study Population}

We recruited the students utilizing a stepwise purposeful sampling approach (table 1). To obtain richer data, we interviewed students from three different cohorts that were exposed to different experiences while practicing their self-assessments (figure 1). All the three interviewed cohorts were students that were in their clinical phase of the curriculum. We interviewed the first two cohorts of students before the separation of self-assessment process from the summative assessment. While we interviewed the third cohort of students after the separation of self-assessment process from the summative assessment in an attempt to assess the summative impact on students' selfassessment practices and the resulting study strategies.

Table 1: Represents the three different cohorts that were invited and interviewed during the research interventions

\section{Cohort 1 (2007-2008) \\ Cohort 2 (2008-2009) \\ Cohort 3 (2010-2011)}

Invited (37 students)

Invited (32 students)

Invited (22 students)

Interviewed (13students) $\quad$ Interviewed (10 students) Interviewed (14 students)

Cohort 1 (G1): All 37 students in the 2007-2008 academic year, were invited to participate in the study. We scheduled the interviews at the students' convenience and based on their study schedules. Students participated in 13 semi-structured individual interviews, after which data analysis revealed data saturation.

Cohort 2 (G2): The same process explained above for cohort 1was repeated with a second group of students (G2) after the implementation of an orientation program on self-assessment and feedback. All 32 students who had recently reached the clinical phase in the academic year 2008-2009, (G2) were invited for interviews. We interviewed ten students, after which data analysis revealed data saturation. 


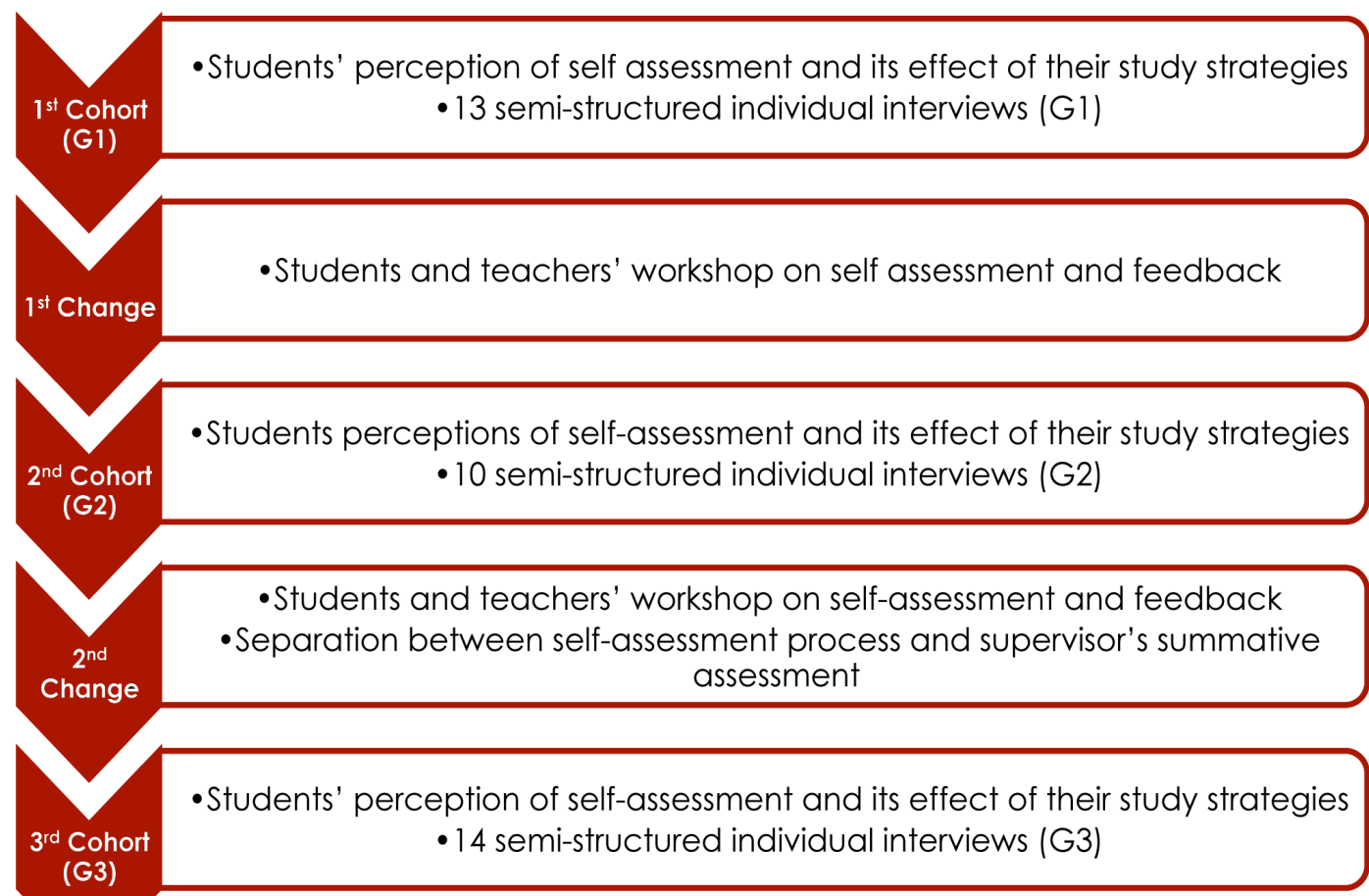

Figure 1: Diagram representing the two implemented changes on the three different cohorts of students included in the research

Cohort 3 (G3): Finally, we invited all the 22 students who had recently reached the clinical phase of the curriculum in the academic year 2009-2010, (G3) to participate in the study. We implemented students and supervisors orientation programs on students' self-assessment and feedback and have administratively separated students' self-assessment process from their supervisors' summative assessment process. We interviewed 14 students, after which data analysis revealed data saturation. No student withdrew consent to participate in the study and no student was interviewed in more than one cohort. 


\section{Data Collection}

The principal author and one of the co-authors interviewed students using openended questions (Appendix 1). These questions were presented in a natural, nonthreatening, conversational and informal setting. We asked the students to talk about their perceptions of their self-assessment, its practice, and factors affecting their selfassessment process and their study strategies. These factors have included the effect of summative assessment and the effect of orientation on self-assessment process on their self-assessment practice. The open-ended questions were based on the research team's observation of students and supervisors' practices of self-assessment and feedback. Each individual interview lasted approximately 25-45 minutes. The interviews were audio-taped, and field notes were taken.

\section{Data Analysis}

A research assistant performed verbatim transcription of the interviews. The transcriptions were analyzed by the principal author and then managed utilizing Atlas.ti (Version 5.2) computer software. Analysis involved line-by-line scrutiny of the transcript and the assignment of relevant codes to text fragments. We identified codes and themes for each interview. These codes and themes were subsequently refined in a longitudinal and transverse cyclic analytical process, moving backward and forward between the interviews. To improve the credibility and transferability of the analyzed data, we performed investigators' triangulation by having a co-author code six interviews independently (two from each group). Similarities and differences in the interpretation of the data were debated until agreement was reached. We performed member checking to achieve further improvement to the credibility and transferability of the data. The results were presented to a group of students who were asked to provide feedback. The approval and financial support of the King Abdullah International Medical Research Centre were obtained prior to conducting the research. 


\section{Results}

We noticed significant similarities in the themes abstracted from the interviews with the three groups therefore, they were analyzed together. Analysis of the data for the three cohorts of students generated three main themes (Table 2). To illustrate how our concept of the effect of self-assessment on student study strategies is grounded in the data, we present quotes from the transcripts. Quotes by different cohorts of students are marked G1, G2 or G3 at the end of the quotes.

\section{Strategic Practice of Self-Assessment}

Students admitted overestimating their self-assessment in an attempt to impress their supervisors and manipulate their decisions to gain higher marks. Even those students who had assessed their own performances correctly or even underestimated their self-assessment were looking for better marks. However, the strategic use of the self-assessment was inconsistent, ranging from overestimation to underestimation of performance. This practice persisted for those who received orientation on how to perform self-assessment, even after eliminating the summative effect of the clinical supervisors' evaluation.

"We are human beings; we are likely to give ourselves better evaluations than what we deserve" (G3).

"I have a self-assessment form already completed in my USB. I rated myself 3 (full mark). Whenever an evaluation form is needed, I print the ready form and only change the date and the supervisor name" (G2).

The students believed in the accuracy of their supervisors' assessment compared with their own assessment. However, the students continued to focus on their marks when they self-assess; they rarely focused on improving their ability to maintain patients' safety, better clinical performance or practice enhancement. The dominant summative mode enhanced students' strategic study strategy or achievement-motivating study strategy. 
Even the third group of students, whose self-assessment was independent of their summative marks for the clinical attachment, thought that their overestimated selfassessment led to higher self-satisfaction and more confidence in patient care,

Table 2: Represents the various themes and codes that were obtained through data analysis

\section{Theme \\ Code}

Feedback and study strategy

Feedback and faculty enhancement

Feedback and students' orientation

Feedback as a drive to learn

\section{Self-assessment and study strategy}

Self-assessment as a drive to learn

Self-assessment and faculty enhancement

Self-assessment and students' orientation

\section{Strategic practice of self-assessment}

Over-estimation of self-assessment

Under-estimation of self-assessment

Accurate estimation of self-assessment

Self assessment and summative effect

Self-assessment and students' self

satisfaction

representing another aspect of an achievement-motivating strategy. The students have isolated their self-assessment practice from the other major component of selfassessment process namely the supervisors' formative feedback. Students' direct interactions with their patients, each other and their clinical supervisors did not change their perceptions of their self-assessment.

"I don't have a fixed strategy for marking myself. Sometimes I give myself outstanding 
marks; sometimes I put an average mark. It depends who my supervisor is" (G2). 'I think the supervisors' assessment is more accurate. People around you can judge your performance more accurately.... Nobody underestimates himself, except a few... I usually overestimate myself because of the marks and GPA..." (G1).

\section{Self-Assessment and Study Strategies}

The majority of the students did not perceive their self-assessment as a drive to learn, and a minority of students saw self-assessment as playing a positive role in their learning. It initially appeared that the summative effect of the implemented self-assessment has limited the positive impact on student learning. However, students persisted in using the same study strategy after eliminating the summative effect of their supervisors' evaluation. Self-assessment, when isolated from supervisor feedback, did not show a positive influence on the practiced study strategies; either it had no effect on students' learning, or it had stimulated an achievement-motivation study strategy in which students put all of their efforts into obtaining higher marks.

"It looks like a good idea, but actually, in real life, it is just a matter of formality.... I don't give it importance. It doesn't change the way I study or approach my patients..." (G2) and "... I don 't think that it is going to make a big difference... I will become a doctor, and I have to be a very good doctor. So I will work hard whether I self-assess or not" (G3). "When I self-assess, this is a trigger for me to learn... It helps me to know where my defects are so I can try to correct them" (G1).

\section{Feedback and Study Strategies}

Students' perceptions of the effect of feedback on their studying and their study strategies were also inconsistent. However, the majority of the interviewed students affirmed the positive effects of feedback on their studying. Some students disagreed with the existence of a positive effect, while a minority of them adopted the extreme view that feedback is occasionally deceiving. 
Self-assessment and students' study strategies

"I follow my supervisors'feedback... but... when it comes to the exams, I sometimes find myself off the right tract... It is sometimes deceiving" (G1).

The majority of students however, believed that formative feedback from supervisor guided them in identifying the necessary knowledge and skills for good patient care. The feedback also identified their knowledge and skills gaps and provided ways through which these gaps could be overcome. Formative feedback had a positive effect on students' performance. It helped them to systematically approach their patients' problems, search for evidence and construct suitable management plans. Therefore, formative feedback stimulated a deeper approach to learning.

"Feedback will help me to identify whether I am on the right track or not, whether I need to improve on some points or just carry on with what I'm doing. For example, if I am managing a patient and I don't know how to approach the case, I waste my time reading irrelevant information. Later, through feedback, I find that I shouldn't have done so" (G1).

Occasionally, negative feedback resulted in student frustration, affront, and loss of confidence in directly encountering patients, and it stimulated a superficial approach to learning. Students commented on the quality of the feedback provided and stressed the need to improve their practice through supervisors training. Moreover, despite students' appreciation to the importance and benefit of the orientation and training they have received on self-assessment and the utilization of formative feedback, students continued to observe inconsistencies in their supervisors' feedback, even after the supervisor enhancement workshop on providing effective feedback. Because of these inconsistencies in the quality of feedback provided, students' practice of study strategies varied between deep and superficial strategies.

"I thought it was going to be a formal, boring meeting, but it was nice. I got a lot from that meeting, and I changed a lot based on it. I realized we were missing the meaning of 
feedback and the effective communication between the students and their seniors" (G2). "Some of the supervisors are very excellent, but they are few. The majority are not good at giving feedback... usually they try to give you a general opinion” (G2).

\section{Discussion}

In the community of clinical practice, several factors were found to contribute to students' perceptions of self-assessment and their practice of study strategies. Students favored training on how to practice self-assessment and the benefits of feedback. However, this training did not change students' strategic practice of self-assessment or their tendency to overestimate themselves. It did not contribute to students' perceptions of patient care or their ability to encounter patients. Regardless of the strategy used for self-assessment whether an overestimation or underestimation of self-performance, the practice was influenced by students' attempts to manipulate their supervisors' summative assessments and receive higher marks or simply to make a good impression. This resulted in an achievement-motivation study strategy and turned the students into grade-seekers. Students valued formative feedback and agreed that it contributed to a deep approach to learning. However, negative feedback was found to result in negative feelings, patchy reading and a superficial approach to learning.

We evaluated the implemented self-assessment program in the community of clinical practice and the resulting student study strategies in relation to Kirkpatrick's four-level evaluation model. We have assessed the degree at which the students have reacted favorably to the self-assessment orientation and implementation. Moreover, we have assessed the degree at which students have acquired the intended knowledge, skills, and attitudes that allow them to practice better study strategy based on their participation in the learning event (Kirkpatrick, 1994). Therefore, we have reached in our analysis the second level of Kirkpatrick's four-level evaluation model (figure 2). In general, students reacted favorably to the implemented education workshops on practicing self-assessment 
and feedback. This finding was not surprising as McDonald and colleagues has obtained similar results by addressing the effect of self-assessment training on the performance of secondary school students in external examinations (McDonald and Boud, 2003). They concluded that the students received the training positively and accepted it as beneficial rather than as an additional burden on their examination year. However, while McDonald studied the academic improvement of students' performance after they have experienced self-assessment, we explored the study strategies practiced by the students because of self-assessment implementation. Similar to McDonald's students, our students acquired the intended knowledge and skills needed to practice self-assessment and utilize feedback. However, unlike McDonald's students, our students did not change their attitudes toward studying and continued to practice preplanned strategies in response to their participation in the learning events within the community of clinical practice.

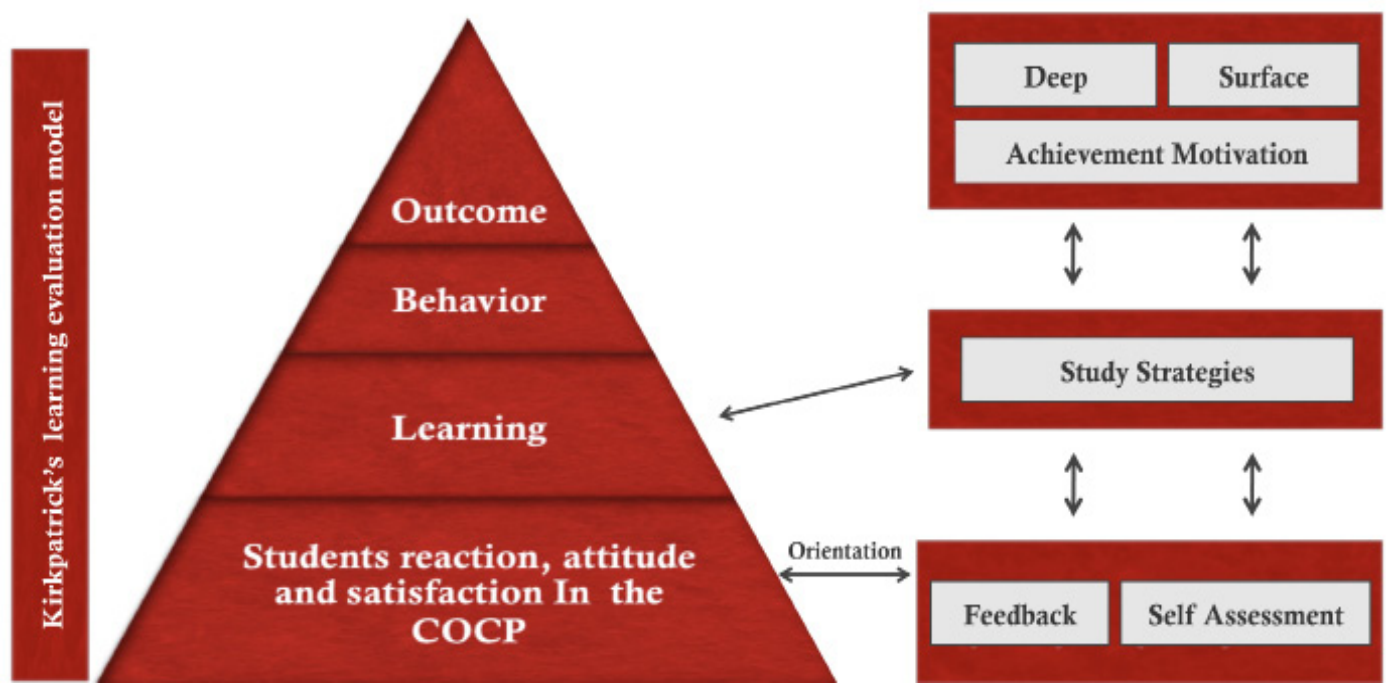

Figure 2: Evaluation of self-assessment related factors contributing to students' study strategies in the "community of Clinical Practice"

COCP: Community of clinical practice 
It appears that education workshops such as those implemented in our study are insufficient to change students' self-assessment practices and their strategic uses of this type of assessment (Shackelton et al., 2009). We should note that the summative characteristic of our assessment and our graduate cohort of students who were more mature than McDonald's students, could have contributed to our students' behavior and their tendency to become grade-seekers (Al Kadri et al., 2011). Interestingly, McDonald and colleagues were unsure of the generalizability of their results, and they discussed factors that may have contributed to the positive impact of the implemented self-assessment for their students. In the view of McDonald, students' high potential for adopting self-assessment practices were influenced by the researchers who were seen as representatives of the examination committee therefore, McDonald's studied group was more motivated than the comparison group.

Implementing self-assessment related feedback in the community of clinical practice depends on supervisors' roles on student learning. To implement this component appropriately, the supervisor's role should extend beyond attending a carefully designed orientation program and excellent training. The supervisor should motivate and lead the knowledge and skills acquired through learning into behavior or more precisely, assist in changing culture. Supervisors should play a major role to guide students toward the appropriate practice of self-assessment, accommodate and utilize supervisor feedback. Their role includes deliberate and consistent knowledge and skills reinforcement (Paes et al., 1994, Sanson-Fisher et al., 2005), consistent work to change the culture and expression of a high level of accountability through highly effective coaching of teaching sessions (Neary, 2000, Kilminster and Jolly, 2000, Sanson-Fisher et al., 2005). The degree to which this reinforcement and coaching occurs may contribute to students' performance improvement, change the culture and obtain positive outcomes of self-assessment (Paes et al., 1994, Tervalon, 2003). 
In our case, the effort in implementing an education/orientation workshop alone was not sufficient to change students' attitudes. We assume that our students' inappropriate experience of self-assessment was not related to failure in establishing a suitable educational workshop rather it is related to failure in changing culture. We cannot neglect the possible contributing factors to our students' attitudes toward self-assessment; students' cultural backgrounds, previous educational backgrounds, personal beliefs and preferences and the accompanied summative assessment that may affect their perceptions of self-assessment, its practice and the resulting study strategies (Tervalon, 2003). In depth exploration of the effect of these factors on students' self-assessment practice is a place for future research.

The most important questions that we would like to answer is the following: Which of the two components of the students' self-assessment process carries the largest effect on students' study strategies?, is it the self-assessment practice or the provided feedback?. By examining our results, we found that despite students' positive perceptions of selfassessment, this positivity was not successful in changing students' study strategies or their strategic approaches to learning; students had a major tendency toward overestimation of their performance. However, appropriately provided feedback was associated with problem identification, correction and therefore, a better approach to learning. It appears from this work that it is likely that the theoretical advantages linked to the self-assessment process are a result of its feedback component rather than the practice of self-assessment isolated from feedback (Eva and Regehr, 2008).

One limitation of this study is that the study population is male. We are not aware of a study that has compared males with females concerning the study strategies resulting of self-assessment practice. Another limitation is the lack of evidence on specific cultural values that may have contributed to the results. Finally, the variability of supervisors' experiences in self-assessment process and feedback might have affected our results. 


\section{Conclusion}

In the community of clinical practice, it appears that of the two major components of the self-assessment process (self-assessment and feedback), feedback is the most effective component for enhancing students' study strategies, leading them toward a deep approach to learning. Feedback component of self-assessment appears to be highly responsible for the documented positive effects of the self-assessment process. The practice of self-assessment on its own, does not appear to be of significant value in enhancing students' learning quality, particularly when applied within a summative context. Further researches that examine the effects of cultural, contextual and personal values on the practice of self-assessment and feedback are needed. 
Appendix 1: Open ended questions that were utilized during the interviews

1. What is your opinion of the value of self-assessment? Why?

2. What is your opinion of the value of feedback? Why?

3. What is your opinion of the value of orientation on self-assessment? Why?

4. How did you benefit from the orientation workshop you have attended? Why?

5. What are the learning opportunity/benefit obtained out of practicing selfassessment? Why?

6. What are the personal uncertainties that can be sorted out by practicing selfassessment? Why?

7. What do you think of having self-assessment implemented as a major assessment tool? Why?

8. On what principle/strategy you are marking (self-assessing) yourself?. Why?

9. Why do you think some are over-estimating/under-estimating themselves?

10. On what principle you think your supervisor marks you?. Why?

11. How do you think you can improve on your self-assessment? Why?

12. How do you think you can benefit from your self-assessment? Why?

13. What about self-assessment as a factor contributing to future safe practice? Why? 


\section{References}

AL KADRI, H. M., AL-MOAMARY, M. S., ELZUBAIR, M., MAGZOUB, M. E., ALMUTAIRI, A., ROBERTS, C. \& VAN DER VLEUTEN, C. (2011) Exploring factors affecting undergraduate medical students' study strategies in the clinical years: a qualitative study. Adv Health Sci Educ Theory Pract. DOI. 10.1007/s10459-010-9271-2.

BLACK, P. \& WILIAM, D. (1998) Assessment and classroom learning. Assessment in Education, 5, 7-74. BOUD, D. (1989) The use of self-assessment in student grading. Assessment and Evaluation in Higher Education, 14, 20-30.

BOUD, D. (1995) Enhancing learning through self-assessment. Kogan Page. London.

BOUD, D. (2000) Sustainable assessment: rethinking assessment for the learning society. Studies in Continuing Education, 22, 151-167.

BURGESS, H., BALDWIN, M., DALRYMPLE, J. \& THOMAS, J. (1999) Developing self-assessment in social work education. Social Work Education, , 18, 133-146.

BUTCHER, A. C. \& STEFANI, L. J. (1995) Analysis of peer, self- and staff-assessment in group project work. Assessment in Education, 2, 165-186.

COLLIVER, J. A., VERHULST, S. J. \& BARROWS, H. S. (2005) Self-assessment in medical practice: a further concern about the conventional research paradigm. Teach Learn Med, 17, 200-1.

DEARNLEY, C. A. \& MEDDINGS, F. S. (2007) Student self-assessment and its impact on learning - a pilot study. Nurse Educ Today, 27, 333-40.

DOLMANS, D. H., WOLFHAGEN, H. A., ESSED, G. G., SCHERPBIER, A. J. \& VAN DER VLEUTEN, C. P. (2002) Students' perceptions of relationships between some educational variables in the out-patient setting. Med Educ, 36, 735-41.

ENTWISTLE, N. \& MCCUNE, V. (2004) The Conceptual Bases of Study Strategy Inventories. Educational Psychology Review, 16, 325-345.

ERAUT, M. (2004) Informal learning in the workplace. Studies in Continuing Education, 26, 247-273.

EVA, K. W. \& REGEHR, G. (2008) "I'll never play professional football” and other fallacies of selfassessment. J Contin Educ Health Prof, 28, 14-9.

FALCHIKOV, N. \& BOUD, D. (1989) Student Self-Assessment in Higher Education: A Meta-Analysis. Review of Educational Research, 59, 395-430.

KILMINSTER, S. M. \& JOLLY, B. C. (2000) Effective supervision in clinical practice settings: a literature review. Med Educ, 34, 827-40.

KIRBYA, N. F. \& DOWNSA, C. T. (2007) Self-assessment and the disadvantaged student: potential for encouraging self-regulated learning? Assessment \& Evaluation in Higher Education, 32, 475-494.

KIRKPATRICK, D. L. (1994) Evaluating Training Programs, San Francisco, Berrett-Koehler Publishers, Inc.

KRUGER, J. \& DUNNING, D. (1999) Unskilled and unaware of it: how difficulties in recognizing one's own incompetence lead to inflated self-assessments. J Pers Soc Psychol, 77, 1121-34.

KRUIDERING-HALL, M., O’SULLIVAN, P. S. \& CHOU, C. L. (2009) Teaching feedback to first-year medical students: long-term skill retention and accuracy of student self-assessment. J Gen Intern Med, 24, 721-6. 
MCDONALD, B. \& BOUD, D. (2003) The Impact of Self-assessment on Achievement: The effects of self assessment training on performance in external examinations. Assessment in Education, 10, 209-220.

MCKEACHIE, W. J. (1990) Research on college teaching: The historical background. J. Educ. Psychol, 82, 189-200.

MCMAHON, T. (1999) Using negotiation in summative assessment to encourage critical thinking. Teaching in Higher Education, 4, 549-554.

NEARY, M. (2000) Supporting students' learning and professional development through the process of continuous assessment and mentorship. Nurse Educ Today, 20, 463-74.

PAES, B. A., MODI, A. \& DUNMORE, R. (1994) Changing physicians' behavior using combined strategies and an evidence-based protocol. Arch Pediatr Adolesc Med, 148, 1277-80.

RAINSBURY, E. \& HODGES, D. (1998) Academic, employer and student collaborative assessment in a work-based cooperative education course. Assessment and Evaluation in Higher Education, 23, 313-325. RAMSDEN, P. (1992) Learning to teach in higher education, London, Routeledge

SANSON-FISHER, R. W., ROLFE, I. E. \& WILLIAMS, N. (2005) Competency based teaching: the need for a new approach to teaching clinical skills in the undergraduate medical education course. Med Teach, 27, 29-36.

SHACKELTON, R., LINK, C., MARCEAU, L. \& MCKINLAY, J. (2009) Does the culture of a medical practice affect the clinical management of diabetes by primary care providers? J Health Serv Res Policy, 14, 96-103.

SOMERVELL, H. (1993) Issues in assessment, enterprise and higher education: the case for self-, peer and collaborative assessment. Assessment and Evaluation in Higher Education, 18, 221-233.

STEFANI, L. J. (1998) Assessment in partnership with learners. Assessment and Evaluation in Higher Education for Health, 23, 339-350.

STERN, D. T., WILliAMS, B. C., GILL, A., GRUPPEN, L. D., WOOLLISCROFT, J. O. \& GRUM, C. M. (2000) Is there a relationship between attending physicians' and residents' teaching skills and students' examination scores? Acad Med, 75, 1144-6.

TAN, K. H. K. (2000) Does student self-assessment empower or discipline students? Assessment \& Evaluation in Higher Education, 29, 651-662.

TARAS, M. (2001) The use of tutor feedback and student self-assessment in summative assessment tasks: towards transparency for students and tutors. Assessment and Evaluation in Higher Education for Health, 26, 605-614.

TERVALON, M. (2003) Components of culture in health for medical students' education. Acad Med, 78, 570-6.

VERMUNT, J. D. (1996) Metacognitive, cognitive and affective aspects of learning styles and strategies: A phenomenographic analysis. Higher Education, 31, 25-50.

VERMUNT, J. D. (1998) The regulation of constructive learning processes. Br. J. Educ. Psychol, 68, 149-171.

WOOD, T. (2009) Assessment not only drives learning, it may also help learning. Med Educ, 43, 5-6.

WOOLLISCROFT, J. O., TENHAKEN, J., SMITH, J. \& CALHOUN, J. G. (1993) Medical students' clinical self-assessments: comparisons with external measures of performance and the students' selfassessments of overall performance and effort. Acad Med, 68, 285-94. 
Chapter 6

\section{Exploring assessment factors contributing to students' study strategies; Literature review}

Accepted for publication as:

Al-Kadri, HM., Al-Moamary, MS., Roberts, C., and van der Vleuten, CPM. (2011). Exploring assessment factors contributing to students' study strategies; Literature review. Med Teach Journal, Saudi Suppliment. In press. 



\section{Abstract}

Background: Assessment steers students' learning is a statement that has been used repeatedly without solid evidence in the literature. This manuscript aims to evaluate the published literatures on the effect of teaching learning environment in particular the implemented assessment on students' learning approaches.

Methods: A literature review was performed on the effect of assessment on students learning approaches. English language literatures were searched in Pubmed, PsycINFO and Medline without restriction to type or date of publication.

Results: Reviewing the literature, the most prominent identified theme was assessment function characterized in summative and formative assessment and general effect of assessment on students learning approaches. The literature review has pointed clearly to the complexity of the relationship between learning environment, students' perceptions of assessment demands, and students' approaches to learning. Many factors (extrinsic and intrinsic) were theoretically proposed to mediate students' approaches to learning in response to their assessment. However, few of these factors were researched in the published literature

Conclusion: Formative assessment is likely to contribute to students' deep approach to learning while summative is likely to contribute to their surface approach. However, these effects are not definite and further research about the complex relationship between assessment and students' learning is required. 


\section{Introduction}

Assessment steers learning is a statement that has been presented by Newble as early as 1983 (Newble, 1988). Since then this statement is viewed as well-proven despite the fact that supportive evidence was quite limited. Historically, adopting the psychometric theories for several decades has focused on factors and indicators, like reliability and validity that are related to assessment quality (Schuwirth and van der Vleuten, 2004). The implementation of such psychometric theory has resulted in summative assessment having higher importance in comparison with formative assessment. Therefore, teachers' test selection was directed to achieve high test quality rather than focusing on the test educational impacts (Schuwirth and van der Vleuten, 2004). Teachers have also consequently avoided practicing a bi-directional process with their students to enhance, recognize and respond to students' learning (Cowie and Bell, 1999). Moreover, they have made little effort to implement formative assessment as a method to help the learning process (Reay and Wiliam, 1999, Black and Wiliam, 1998b). Assessment then became a method to rank students or decide their pass or fail rather than an instrument to enhance their learning. Its influence was mainly on assessment of students learning rather than assessment for students learning enhancement.

In contrast to the psychometric theory, assessment for learning moved the educational focus from an individual instrument into programmatic assessment where we tend to use multiple assessment instruments each of which is directed to cover particular aspect of the intended educational objectives (Schuwirth and Van der Vleuten, 2011, van der Vleuten and Schuwirth, 2005). In this approach, the old assessment tools are not replaced with the new ones, rather they are supplemented with tools that focus more on the tests educational impact (Prescott et al., 2002) and utilize formative assessment to enhance students learning. Assessment for learning was then thought to direct students' studying towards deep approach to learning. 
Research into student learning initially built up evidence about the relationships of motivation and study methods with academic performance (Biggs, 1970, Biggs, 1976, Entwistle and Entwistle, 1970, Schmeck et al., 1977). Researchers stressed the importance of the student's own effort and application in determining levels of academic achievement that shifted the responsibility for high achievement to student's shoulders (Biggs, 1970, Biggs, 1976, Entwistle and Entwistle, 1970).

The initial learning style inventories have emphasized the relative stability of students' learning approaches and were used to predict students' future academic performance (Biggs, 1970, Biggs, 1976, Entwistle and Entwistle, 1970, Schmeck et al., 1977). Based on the early inventories students' learning approaches were found to reflect three basic learning models (Broadbent, 1966): (1) surface learning, involving "repetition of analyses already carried out" and usually directed at reproducing information; (2) deep level learning, using "a greater degree of semantic or cognitive analysis" usually aimed at gaining insight and (3) "assessment driven category", named a strategic approach to learning. Researches on students' learning approaches have progressed to focus more on the effect of the teaching-learning environment (Biggs, 1999) and have investigated the different ways students interpret the requirements of a task within a specific learning context (Marton and Saljo, 1997). Students learning approaches were found to be unstable, swinging between superficial, deep and strategic approaches as a result of different contextual and personal factors. Moreover, inventories have emphasized students' self-conscious reflection on learning, drawing on the ideas of "meta-cognition" and "self-regulation" (McKeachie, 1990, Vermunt, 1996, Vermunt, 1998). Students learning approaches categorizations were then modified to deep, reflective and elaborative approaches and superficial with serial, reiterative, or rehearsal approach. A third approach of learning that describes methodical, well-organized studying linked to effort that is done by the students based on certain motivation and aiming 
for certain achievement such as passing exam or achieving high scores or leaving good impression and obtaining certain recommendation. This particular strategy is called effort and achievement motivation learning strategy and has replaced the strategic strategy approach.

Several researches have been published aiming to explore the relation between assessment and learning. However, until recently, literature was not firmly sure of the effect of assessment on students' learning (Norman et al., 2010). The aim of this literature review is to explore and synthesize the published literatures on the effect of teaching learning environment in particular the implemented assessment on students' perception of their learning and their study strategies.

\section{Methods}

A literature review was carried out to identify and analyze results of what have been published on effects of assessment on students' approaches to learning. The search was conducted through peer reviewed journals and the used searching terms were assessment and study strategy, assessment and learning approach, assessment and deep learning, assessment and surface learning. Pubmed and Medline engines were searched as well as PsycINFO. The only restriction was applied to English language and human literature. Otherwise, there was no restriction to the type of articles or the date of publication. References of all relevant articles were reviewed and experts in the field were contacted to explore the presence of relevant work to be reviewed.

As the collected data were heterogeneous, the identified literatures were clustered under the common themes that were recognized. Therefore, the identified literatures were synthesized under the prominent theme of "summative" and "formative" assessment. Further literatures that have discussed the "general effect of assessment on students learning" were synthesized under this title. 


\section{Results}

The majority of identified literatures discuss general assessment factors contributing to students' approaches to learning (Al Kadri et al., 2011b, Al Kadri et al., 2009, Gijbels et al., 2008, Kadri et al., 2011, Nijhuis et al., 2005, Santy and Mackintosh, 2000, Scouller, 1996, Segers et al., 2008, Segers et al., 2006, Shen et al., 2008, Struyven et al., 2006, Tang, 1994, Tiwari et al., 2005). They also focus on various aspects of either summative assessment (Cilliers et al., 2010, Cilliers et al., 2011, Leung et al., 2008) or formative assessment (Rushton, 2005, Tillema, 2001, Baeten et al., 2008, Koh, 2008, Black and Wiliam, 1998a, Sluijsmans et al., 2002) or were in the form of literature review (Broekkamp Hein et al., 2007, Dochy et al., 2006).

A small number of literatures addressed the swinging study strategies practiced by the students between surface and deep approaches to learning e.g. (Al Kadri et al., 2011b, Mattick and Knight, 2007, Mattick and Knight, 2009, Newble and Jaeger, 1983). Moreover, there were scarce literatures that assessed circumstances or conditions under which students reacted to their assessment and changed their learning approach whether towards deep or surface approach to learning e.g. (Kadri et al., 2011, Al Kadri et al., 2011b, Al Kadri et al., 2009). Furthermore, there were even more scarce literatures that have discussed the role of assessment in driving students' learning in a clinical teaching context (Al Kadri et al., 2011a, Al Kadri et al., 2011b, Al Kadri et al., 2009, Kadri et al., 2011).

Broekkamp (Broekkamp Hein et al., 2007) has developed a theoretical model that integrates various factors which seem relevant to learning strategy adaptation in students' test preparation, including the teacher's intended task demands, students' perceptions of these demands, students' personal goals for learning, and their ability to adapt and implement strategies. The basis of this theoretical model is the construction of strategy adaptation as used in basic cognitive psychology research (Schunn and 
Reder, 1998, Luwel et al., 2005). There was no reliance on medical education literature or on literatures that have discussed students' assessment in a clinical environment.

In this theoretical framework, it was acknowledged that study strategy adaptation might include adaptation to both external and internal task characteristics. While the external task characteristics have included the task implementation environment and its context, internal task processes have included students' ability to adapt various learning strategies, their perception of the task demand and task disposition. In fact research on strategy adaptation in test preparation reported diverse results (Broekkamp Hein et al., 2007). These results varied across disciplines and between experimental and authentic researches. They also differed with the variations of students' grades, between experimental and authentic research environments, different data collection methods and variation in the environmental and individual conditions under which students were expected to adapt their learning strategies to task demands.

Recent research published by Al Kadri et. al. (Kadri et al., 2011, Al Kadri et al., 2011b, Al Kadri et al., 2009) have validated few areas of applicability of factors presented in Broekkamp's theoretical framework in a clinical education field. In their work they have identified ways through which these theoretical factors affect students' approaches to learning, the direction of these effects, contributing factors and how and why they affect students' strategy adaptation. They have assessed various extrinsic factors such as education and students' culture, curriculum objectives, clinical supervisor and supervision and intrinsic factors such as task demand, motivations and task goals. What was prominent in Al Kadri's findings is that though assessment was found to drive students learning, this was not always a positive drive. Students' learning approaches in response to their assessment were not stable and were swinging between surface, deep and achievement motivation strategies. These swinging effects have varied between studied institutions, different studied students, the students themselves and the assessment function whether summative or formative. 


\section{Summative Assessment and Students' Approaches to Learning (table 1)}

Students cannot escape the impact of summative assessment (Boud, 1995). The design of such assessment is more typically informed by psychometric rather than learning considerations, i.e. usually it is designed to assess students' learning rather than to enhance it. In contrast to formative assessment, summative assessment is used to measure students' achievement or failure and judge their performance. It has a dominant effect on students' learning (Knight, 2001). However, the degree of its impact on students' learning is strongly correlated with the magnitude of the severity of consequences associated with its implementation (Al Kadri et al., 2011b, Cilliers et al., 2011).

Cilliers (Cilliers et al., 2010, Cilliers et al., 2011) has published two exploratory studies aiming to probe the mechanisms by which assessment has impacts on learning. He focused on how various dimensions of summative assessment bring about its influence on students' learning. A grounded theory was adopted to conduct unstructured interviews with students in a South African medical school. In these two researches it was noted that learning behaviors of the students are influenced by their appraisal of the impact of assessment and appraisal of their learning response. Moreover, it was found that the negative effect of summative assessment on students learning is overwhelming and outweighs other aspects of assessment effects if not designed in harmony with the overall program. Al Kadri et. al. (Al Kadri et al., 2011b, Al Kadri et al., 2009) have assessed qualitatively students perceptions of their implemented assessment and the resulting study strategies. In these researches they have indicated that summative assessment is strongly influencing students learning leading to more tendencies towards surface approach to learning or in the best case scenario it leads to achievement motivation study strategy rather than directing students toward deep learning approach. However, Al Kadri has indicated that the effect of assessment is not standing alone and it is strongly affected with other different contextual factors such as students' cultural background, job opportunities 
and health care system. For example, students from multi-culture background such as those living in Sydney, Australia and those who were within health care setup that rely mainly on outcome based assessment rather than students' summative assessment results were more adaptive to summative assessment induced stress and considered it as a positive drive to their learning. On the contrary, students who were affected by summative assessment performance consequences were under more stress that has lead to patchy and superficial learning and turned those students into mark hunters. The results of both authors Al Kadri and Cilliers are stressing on the need to accommodate any assessment program within the students' culture and educational environment. In their opinion, such accommodation will enable health educators to get the best of their students' perceptions and direct their studying positively towards deeper approach to learning.

Leung et.al (Leung et al., 2008) have assessed the role of appropriate assessment program design for nursing students on promoting students' deep approach to learning. They used a multiple choice question tool to assess its summative use on nursing students' learning approaches. Leung noticed a general increase in the surface approach and a decrease in the deep approach to students' learning between the pretest (beginning of course) and post-test (completion of the course). It was found that the multiple choice question assessment tool enhanced students' surface approach to learning. Whether this surface approach was affected by the assessment tool used or the summative effect is not clear. However, Leung (Leung et al., 2008) proposed that it was likely that various other contextual factors had contributed to this result and affected students' approach to learning. These "theoretically suggested" factors or un-experimented factors namely students' work load, curriculum design and teaching methods" were part of (Broekkamp Hein et al., 2007) extrinsic factors that were presented in their theoretical framework. To justify Leung and Broekkamp's suggestions of the responsibility of these factors on the effect of assessment on 
students' approaches to learning in medical education setup, further research is needed.

\section{Formative Assessment and Students' Approaches to Learning (table 2)}

Black and Wiliam (Black and Wiliam, 1998a) in their review article, examined over 250 studies on formative assessment. Their meta-analysis of these articles revealed that formative assessment resulted in positive benefits to learning and that good feedback could make a considerable difference to the quality of students' learning. Moreover, Santy and Mackintosh (Santy and Mackintosh, 2000) in another review that assessed the learning process in nurses education, seconded Black and Wiliam's results and recommended that formative assessment be considered as an integral part of students education rather than as a means of measuring ability. They have emphasized the use of different methods to assess students formatively with the intention of engaging these students in learning activities that address gaps in knowledge and provide feedback on their learning achievement. Therefore, they have recommended the use of assessmentto enhance students' learning rather than to assess their achievement. Further review onthe effect of formative assessment on students' learning was done by Koh et.al. (Koh,2008) who have summarized the benefits of formative assessment implementation to (1) students' development of deep thinking, (2) maintenance of students' motivation and (3) enhancement of students' self esteem and encouragement of self regulated learning. However, while confirming both Black's and Santy's results in formative assessment positive outcome on students' learning, Koh has emphasized the role of students and teachers' understanding of both assessment environment and assessment process to achieve desired educational results. Teachers' awareness of the interrelationship between the three areas of "assessment, curriculum, and pedagogy" was further stressed by Klenowski (Klenowski, 1996) and was identified to influences students' deep approach to learning. Teacher's personality, availability, training and effectiveness as a role model carried a positive impact on students' approaches to learning. Despite this strong emphasis on the role of teachers in students' learning, when teachers have a simple rather than conventional idea of 
stimulating students' studying, they prefer summative assessment to formative as afactor that leads to deeper approach to learning. Those teachers do believe that the only and first priority in designing an assessment program is to serve the purpose of students' accountability, ranking and certifying competence.

On the other hand they deny its remarkable effect on promoting students' learning. It appears that teachers' education, experience and cultural backgrounds have an influence on how they perceive different assessment programs and how they respond to the implemented assessment whether summative or formative (Al Kadri et al., 2011b, Al Kadri et al., 2009, Kadri et al., 2011).

In fact, most of the literatures on formative assessment are linked more strongly to teaching rather than learning (Tillema, 2001, Rushton, 2005). Edstrom et al. (Edstrom et al., 2006) suggest that students' perception of assessment depend less on how it is actually designed, but more on how it is presented to them. However, some teachers view assessment as a process they go through after finishing their teaching rather than something central to learning (Koh, 2008). As teachers can alter the students' perception of assessment, it is worthwhile to spend enough time to explain clearly to these teachers the intention of formative assessment and their significant influence on students learning approaches.

Although formative assessment had been found to be linked to students' deep approach to learning, based on Rushton's synthesis of educational literature to healthcare (Rushton, 2005), it was questionable if the required paradigm shift in assessment culture has occurred. Rushton's analysis suggests that further changes are required in assessment practice. These changes should enable effective development of formative assessment involving the consideration of teaching and learning strategies, in particular, the provision of feedback. 


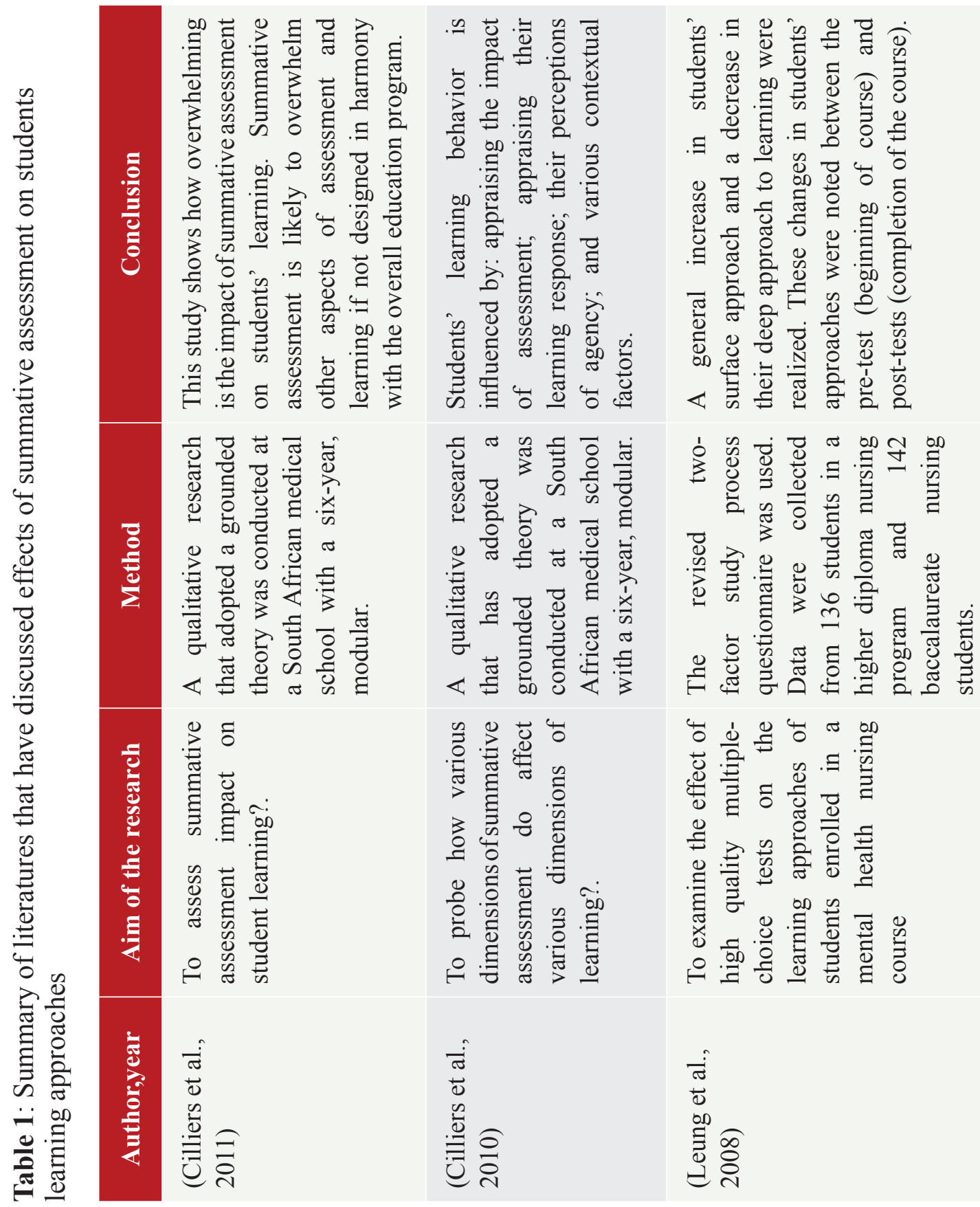




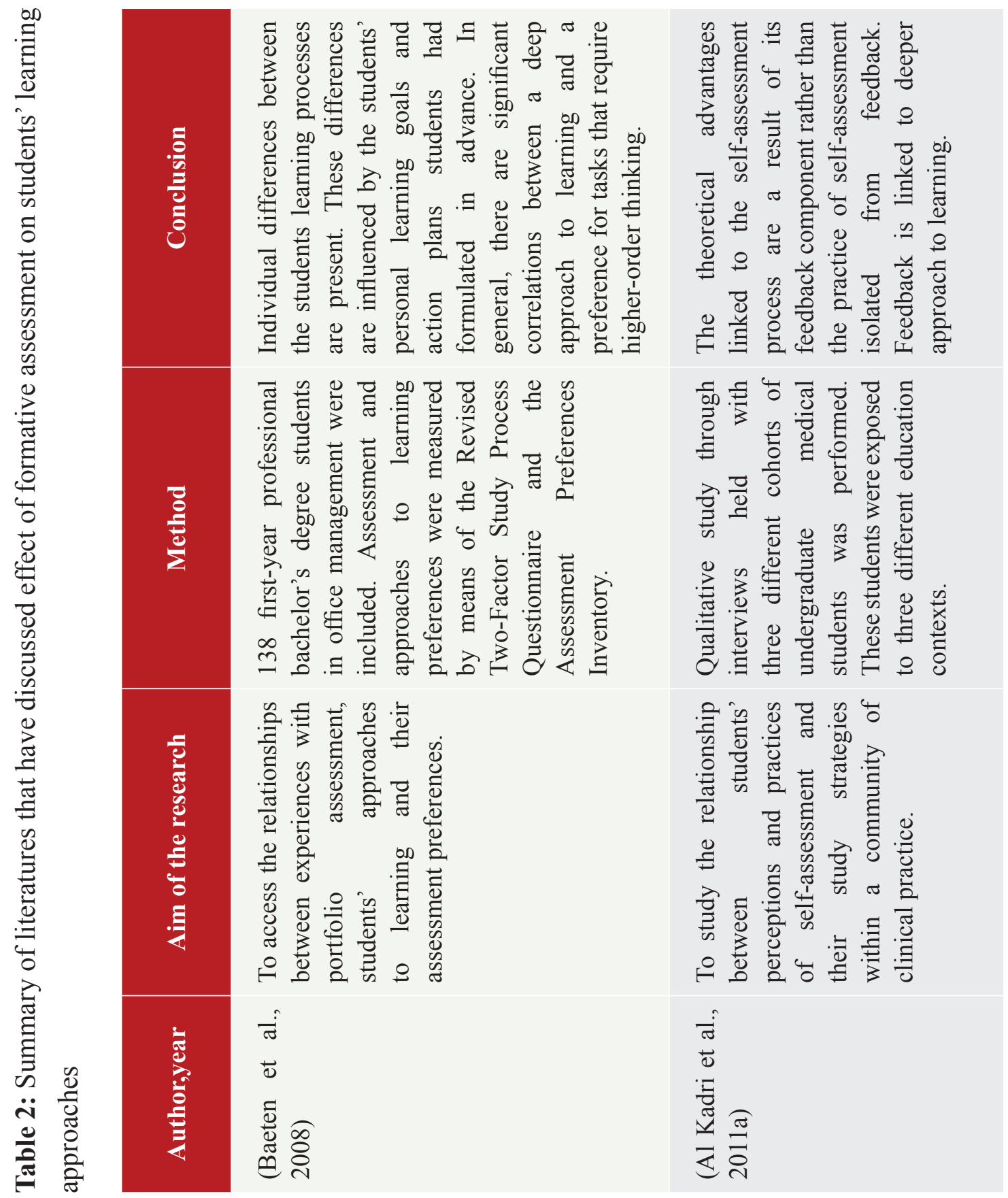




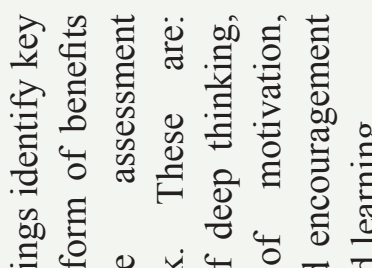

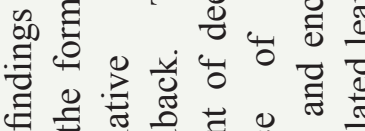

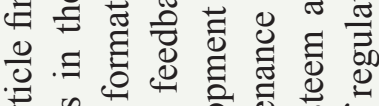

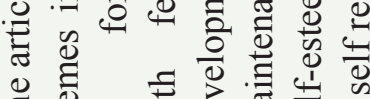

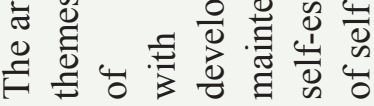

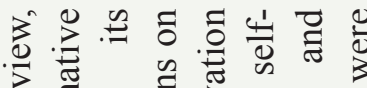

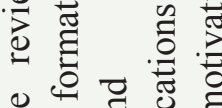

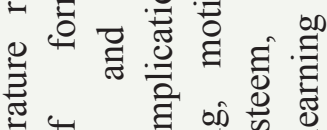

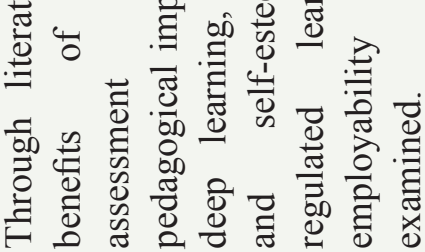

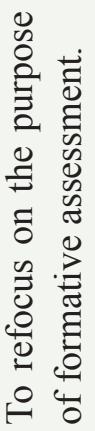

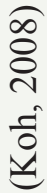

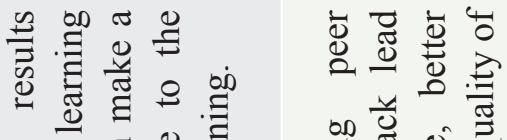

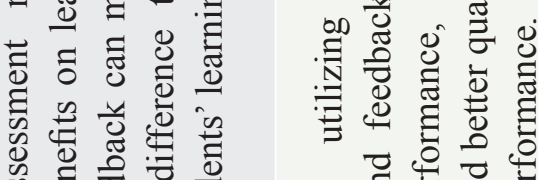

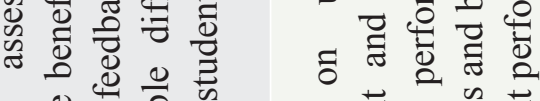

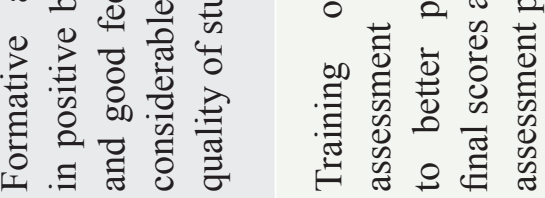

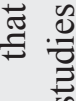

ํํำ

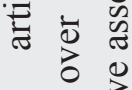

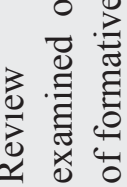

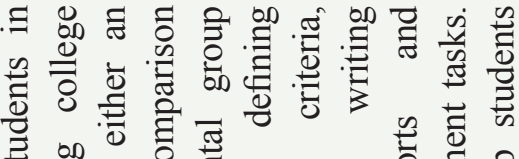

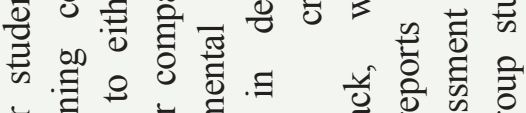

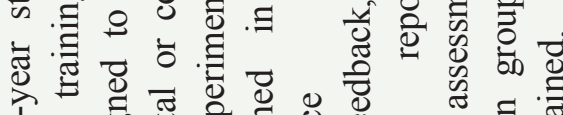

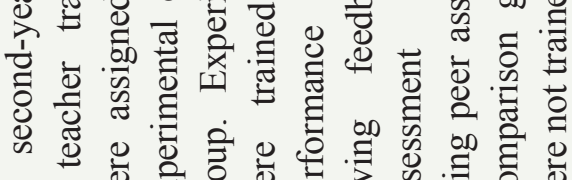

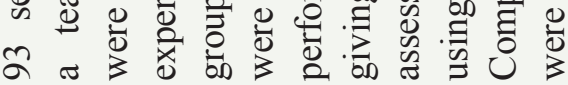

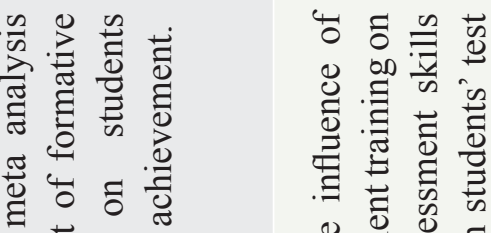

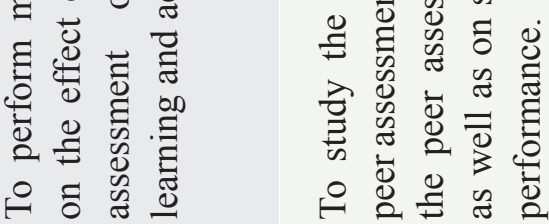

$\varpi$

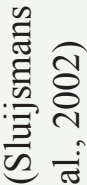




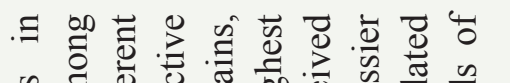

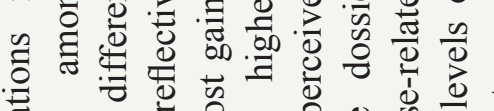

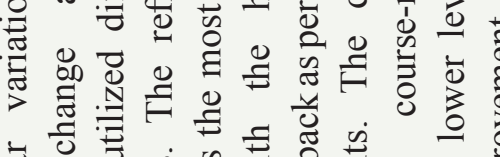

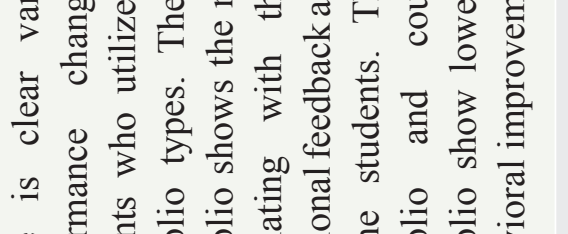

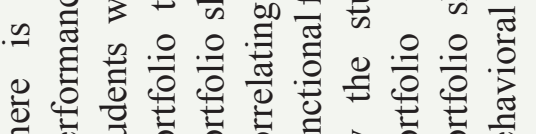

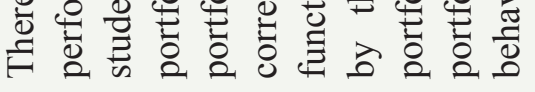

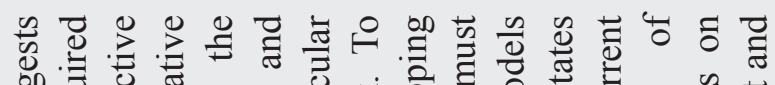

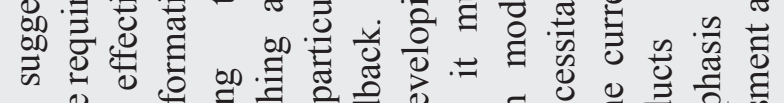

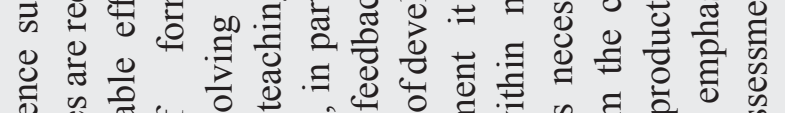

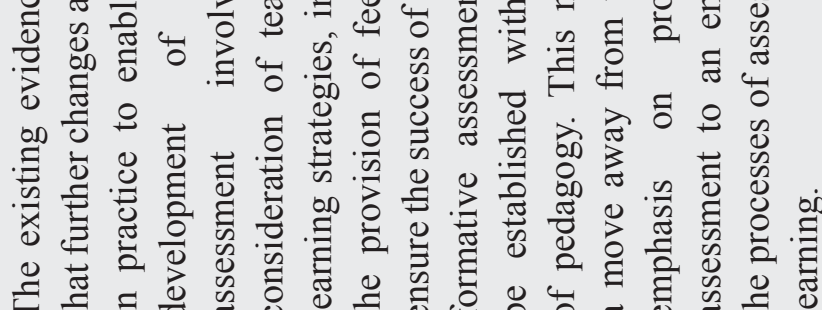

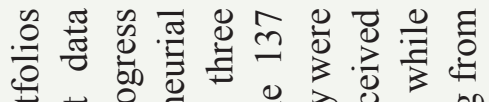

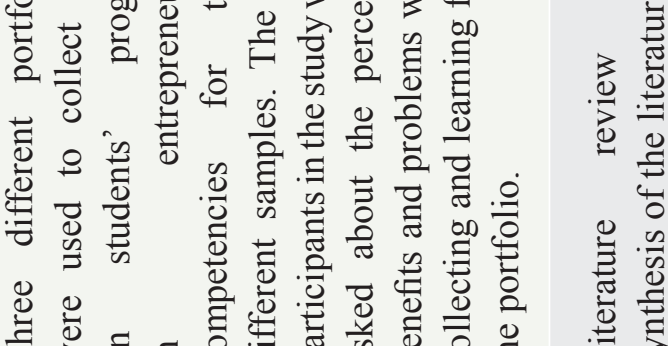

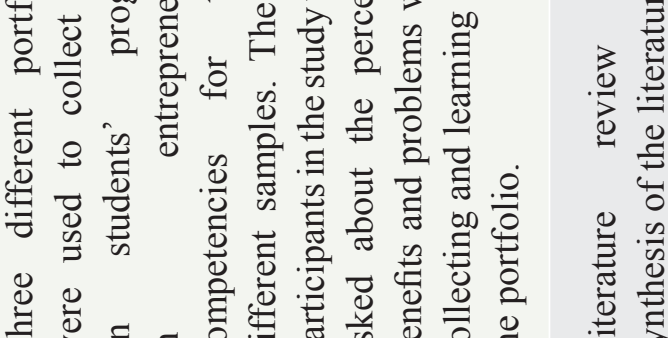

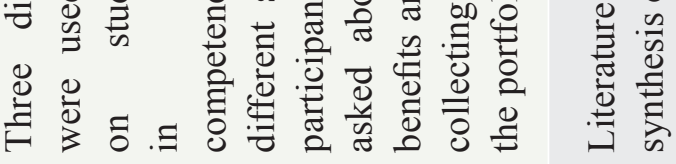
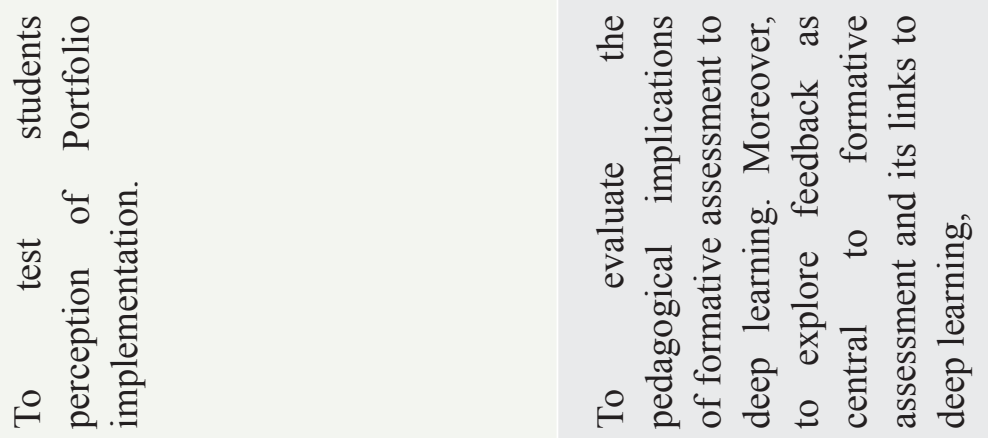

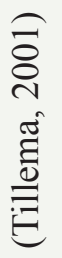

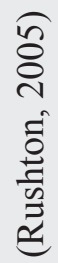


Three years after Rushton's plea, Baeten et. al. (Baeten etal., 2008) published their experiment on the dynamics of students' approaches to learning and their assessment preferences in a portfolio-based learning environment. It appears that despite Rushton's recommendations, similar findings were achieved by Baeten. The cultural change did not occur and Baeten's students' learning processes were goal-oriented and were individually different because of students' personal learning goals and action plans. A similar finding was reached later on by Al Kadri et. al. (Al Kadri et al., 2011a, Al Kadri et al., 2011b) where educational culture and students' and teachers' beliefs of the role of assessment have significantly affected students' perception of formative assessment and their study approaches.

Overall, it must be stated that literatures highlight many claims regarding the positive effects of formative assessment on learning. These claims might be "overstated and under-theorized" particularly when considering students' approaches to learning (Torrance and Prayor, 1998). Further work is recommended to put the existing theories into practice. Moreover, it appears that more emphasis is needed on the role of culture and culture change to achieve the positive learning effects of formative assessment. This change is required as a pre-requisite to formative assessment implementation.

\section{General Assessment Effects on Students' Approaches to Learning (table 3)}

Students might have a predisposition or preference to either deep or surface learning approaches (Biggs, 1993). This preferred learning approach can be modified by the learning environment for individual courses or for particular tasks (Ramsden, 1984). For example students may vary in their approaches to learning when trying to cope with assessment tasks. Learning approaches therefore, are not stable (Marton and Saljo, 1976, Al Kadri et al., 2011b). The direct effects of the students' general beliefs on the cognitive demands of assessment and students' learning approaches were investigated by Segers (Segers et al., 2008). Segers found that students' perception of the deep-level demands have mediated their adoption of deep approaches to learning. For some students deep 
approach to learning is perceived as requirements for all assessment conditions (Tang, 1994). However, this perception is not always paralleled by similar change in students' approach to learning (Gijbels et al., 2008). These approaches are affected by students' pre-intended learning strategies at the beginning of a study course (Gijbels et al., 2008, Segers et al., 2006, Nijhuis et al., 2005). This intention is under influence of various factors including assessment that may strongly affect the way students prepare for their tasks (Scouller, 1996, Struyven et al., 2006, Broekkamp Hein et al., 2007). In general when students plan to learn and understand, they tend to use a deep approach to learning, but when they prepare for their exams, regardless their perception of learning approaches, they are likely to use less of deep approach to learning and more of surface approach to learning (Tang, 1994). This is found to be true even with the introduction of new modes of assessment (Struyven et al., 2006) that requires more deep thinking and analytic approach and it is also true even with the change of learning environment (Shen et al., 2008) through education, explanation and orientation on the benefits of certain assessment tool or certain learning approach (Al Kadri et al., 2011a). These facts add more priority to the need for changing education culture in order to enhance students learning.

\section{Conclusion}

Even after almost a century of research, efforts to positively influence learning through assessment do not always yield to encouraging results. This reflects how little we know about the complex relationship between assessment and students' learning and highlights the urgent need to conduct further research to explore this area.

The previous literature review has pointed clearly to the complexity of the relationship between the learning environment, the students' perceptions of assessment demands, and students' approaches to learning. This complexity indicates that many factors (extrinsic and intrinsic) are mediating students' approaches to learning in response to their assessment. It appears also that drawing a firm conclusion on different assessment factors contributing to students' learning approaches is difficult. 

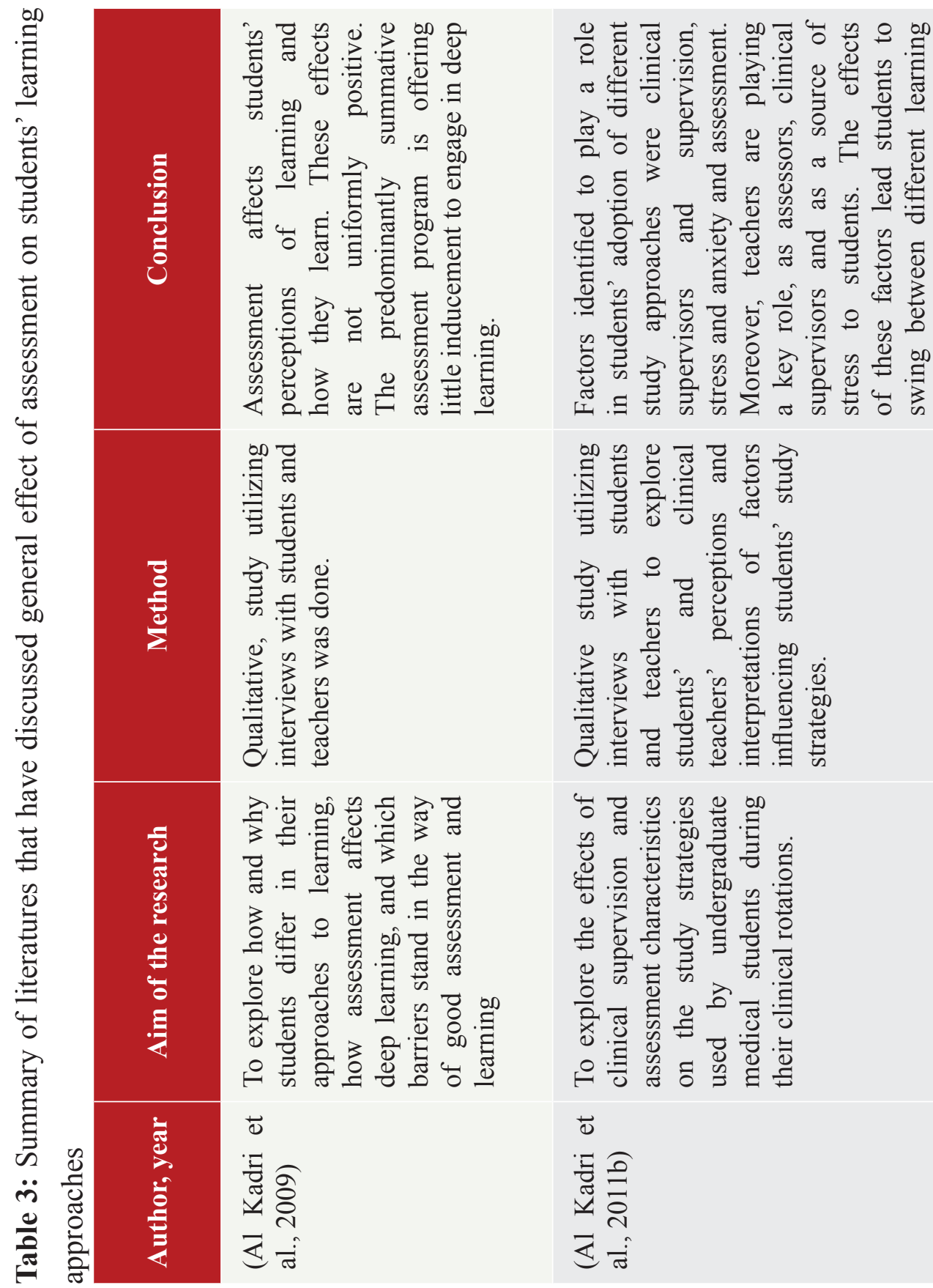

స广

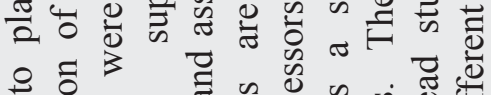

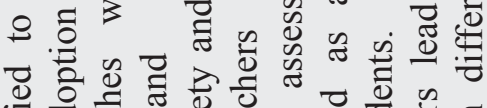

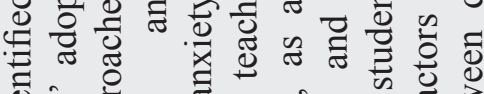

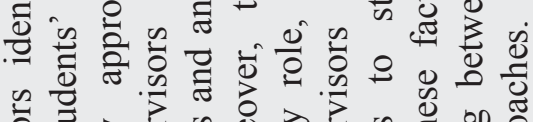

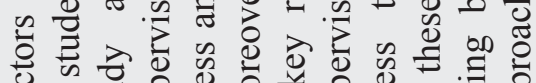

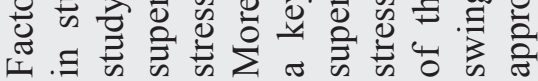

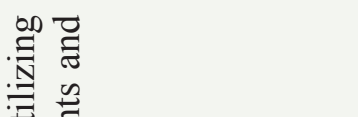

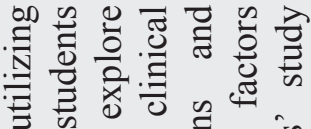

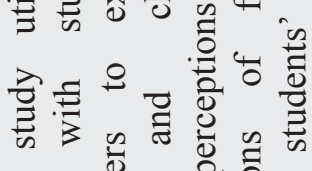

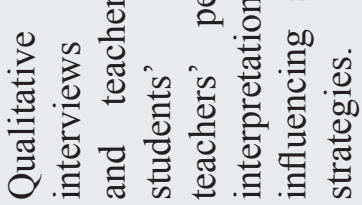

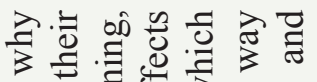

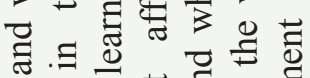

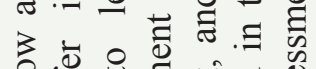

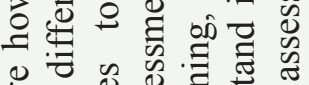

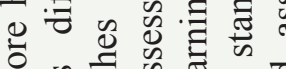

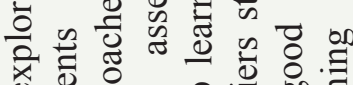

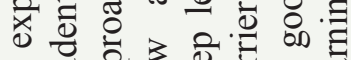

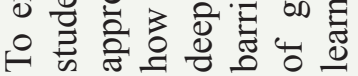

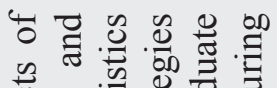

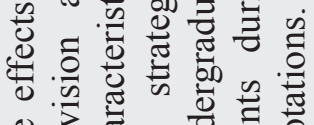

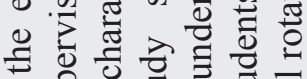

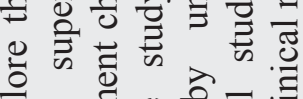

훙 छٓ

으의

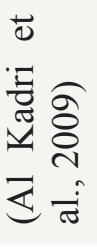

$\bar{\nu}$

폰을

ङ 


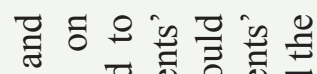

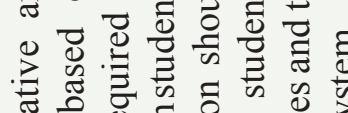
哲

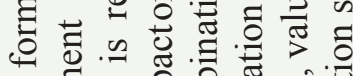

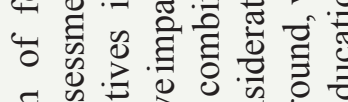

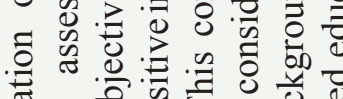

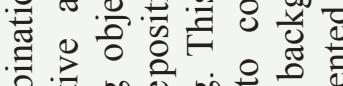

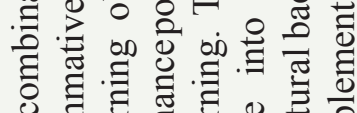

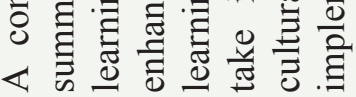

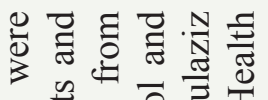

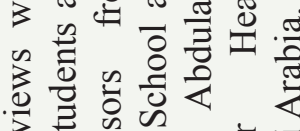

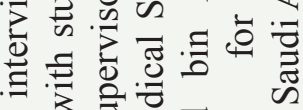

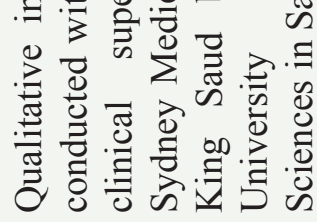

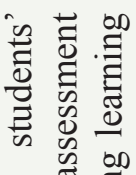

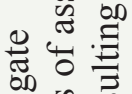

空

क्ष

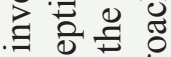

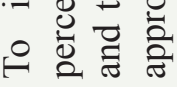

बं

$\overline{0}$

节

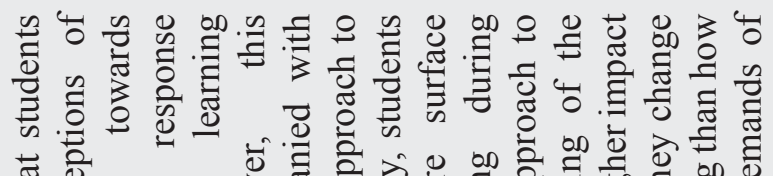

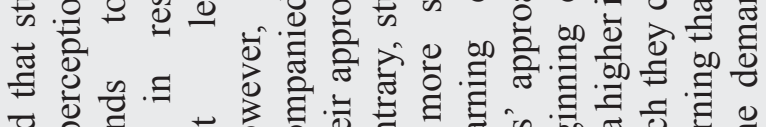

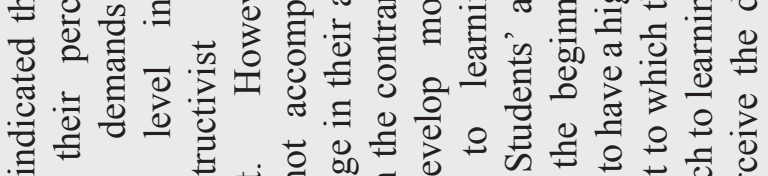

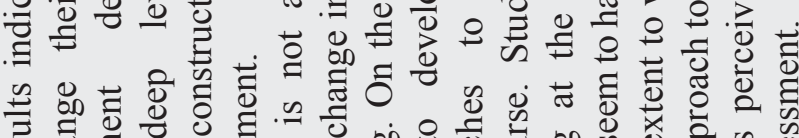

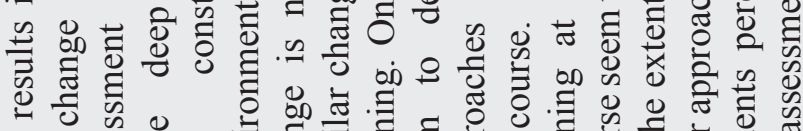

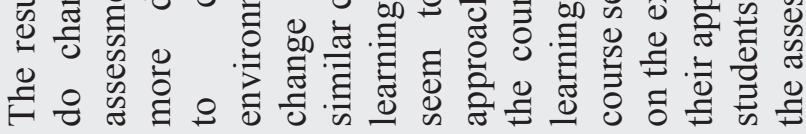
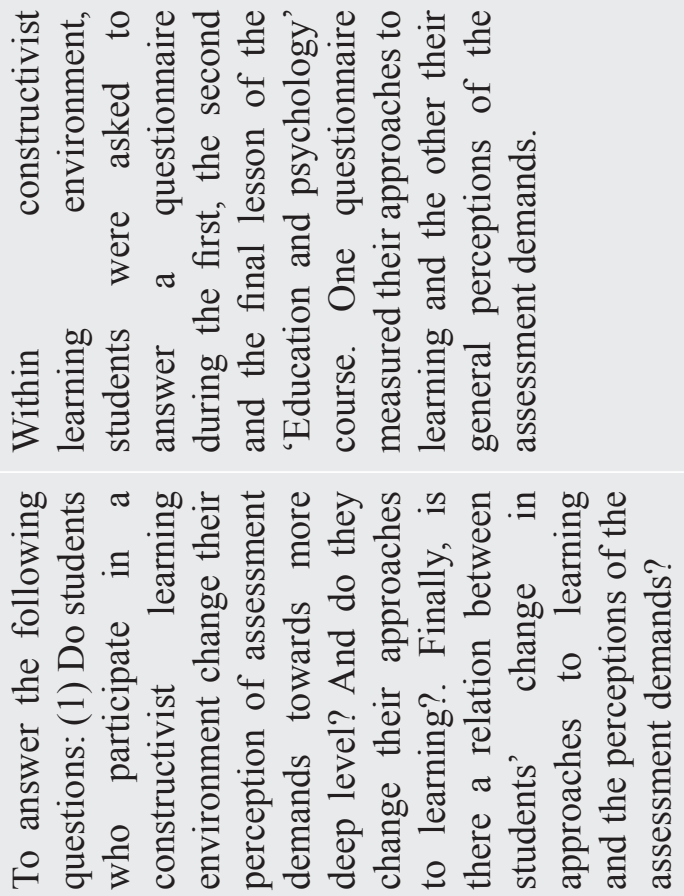

$\varpi$

递竞 


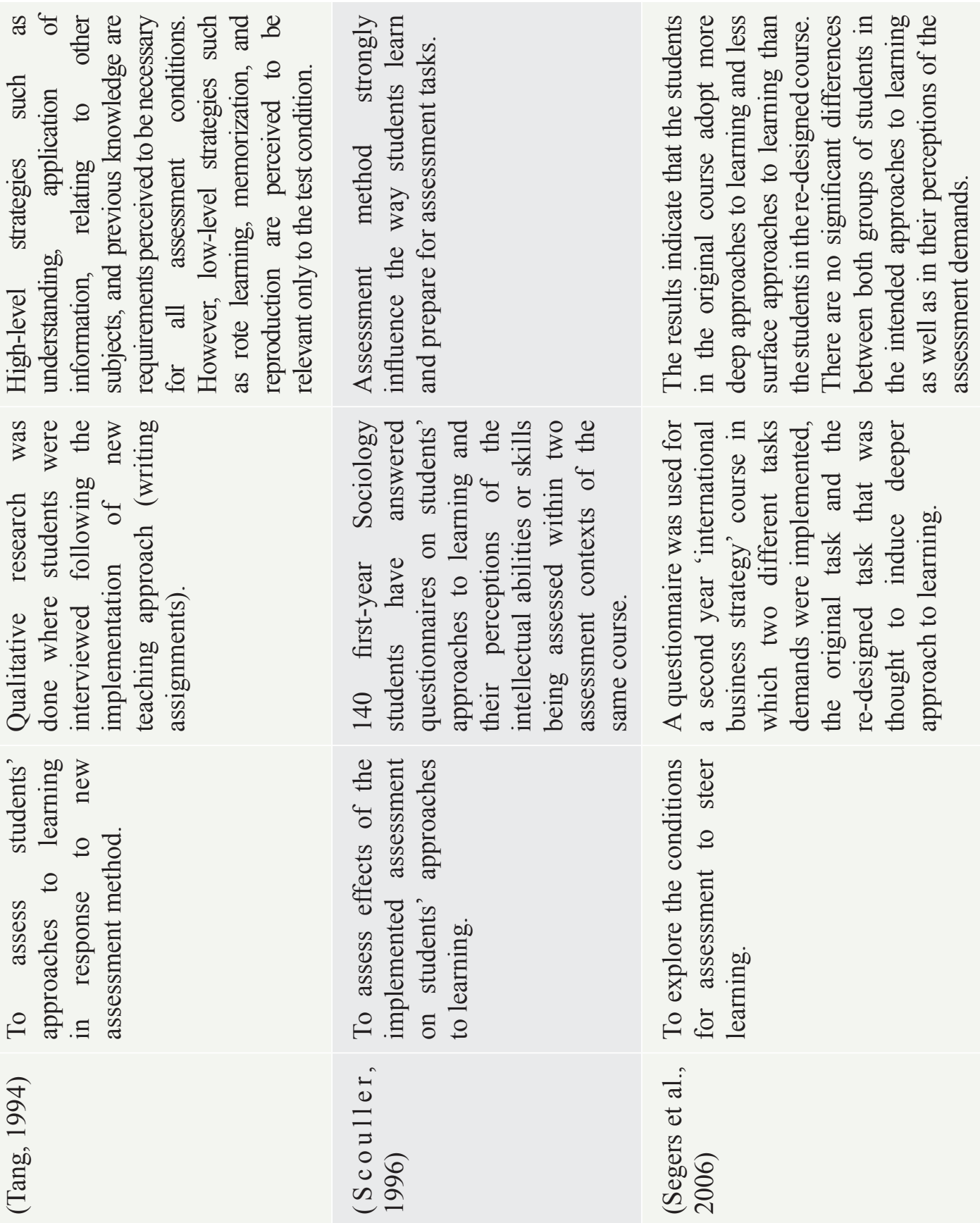




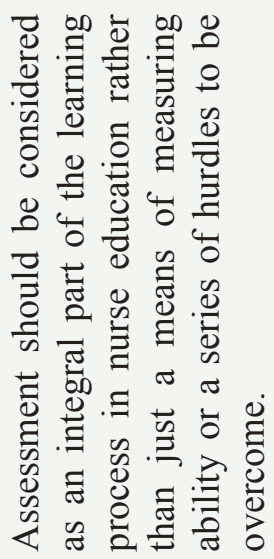

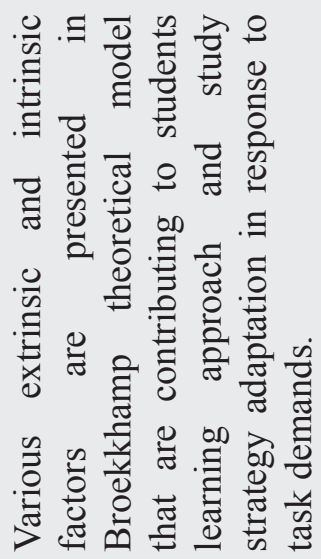

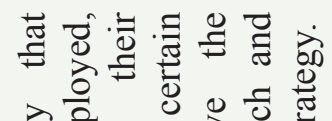

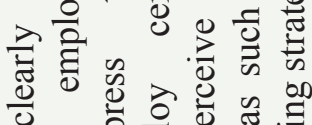

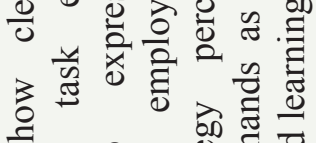

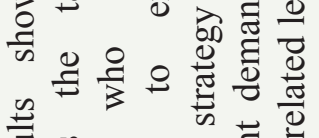

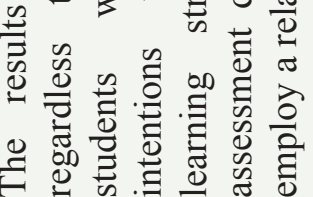

छ

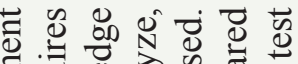

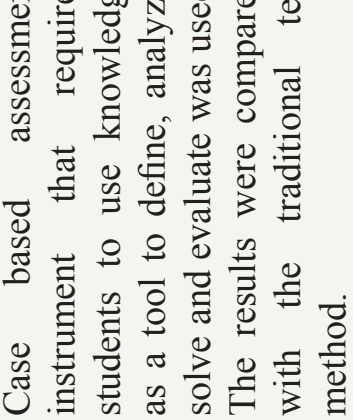

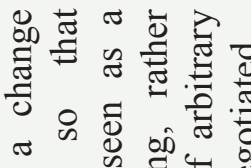

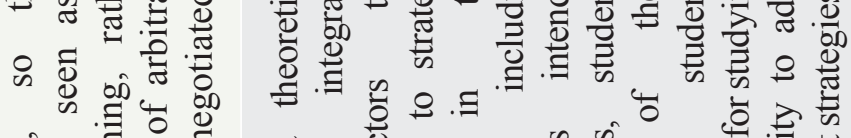

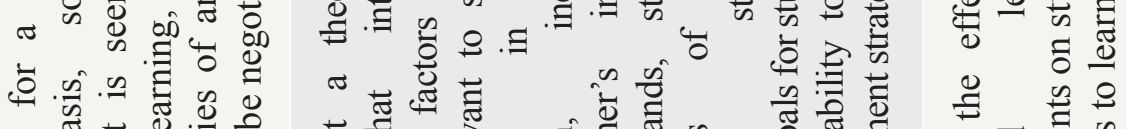

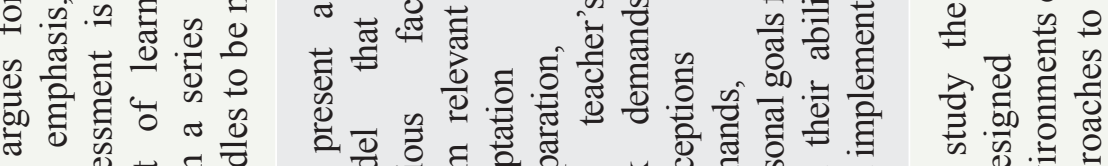

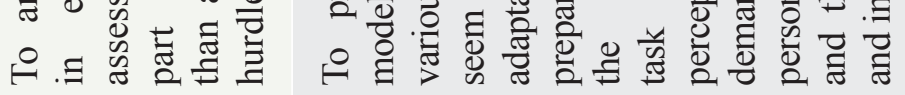

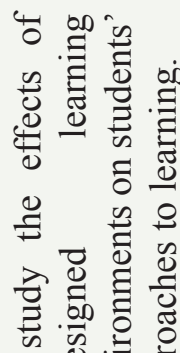

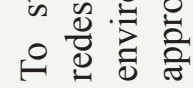
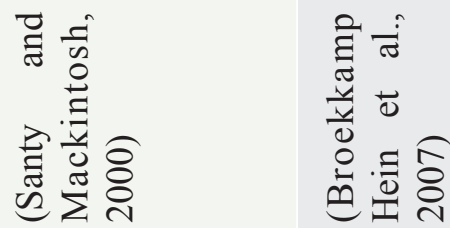

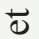

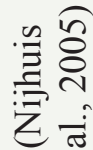




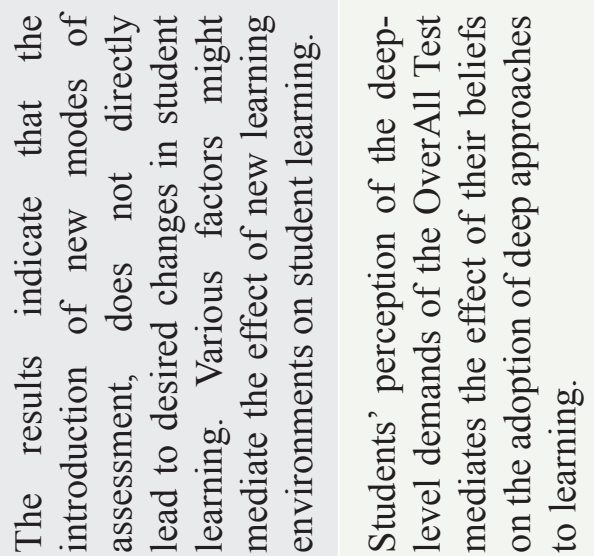

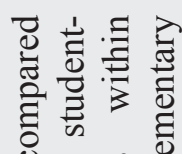

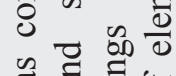

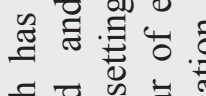

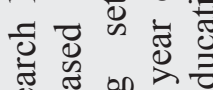

造

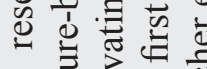

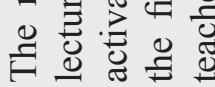

岁驾

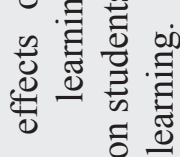

$\stackrel{0}{\square}$

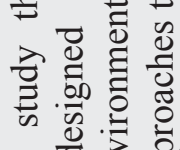

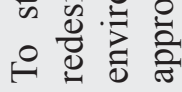

范

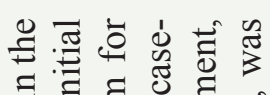

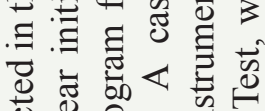

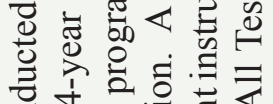

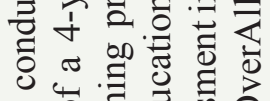

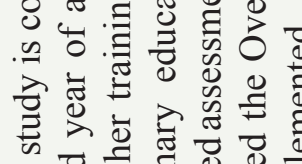

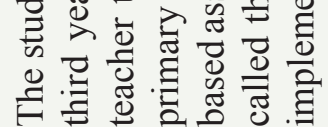

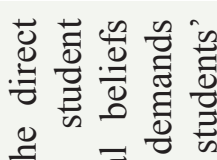

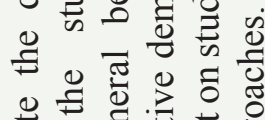

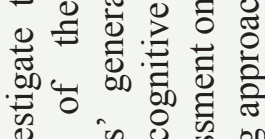

क⿺辶大

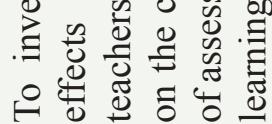

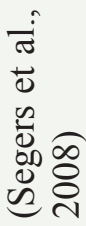

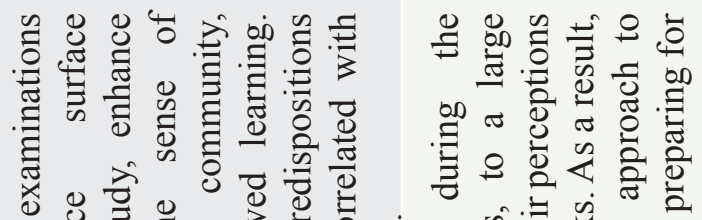

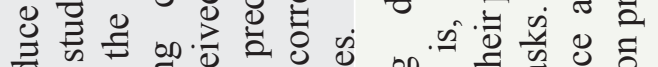

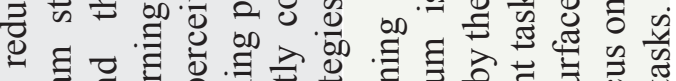

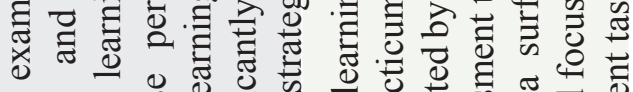

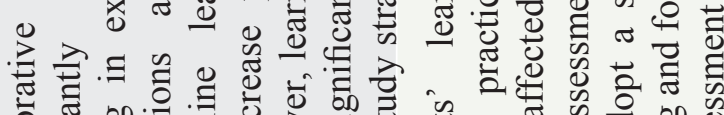

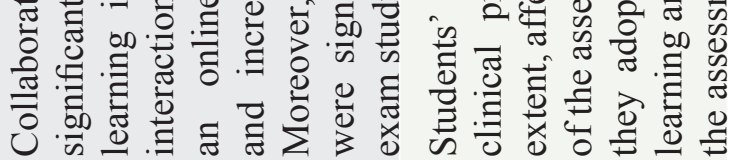

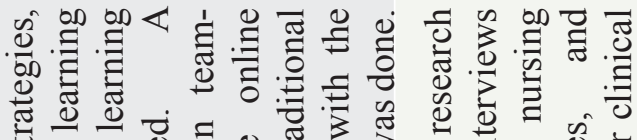

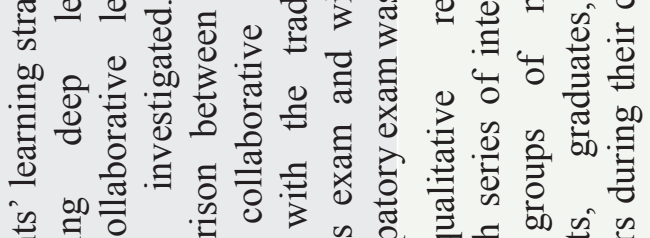

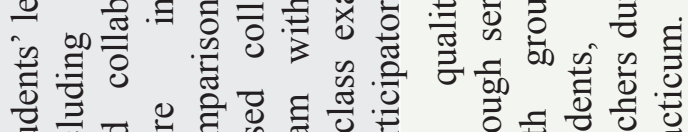

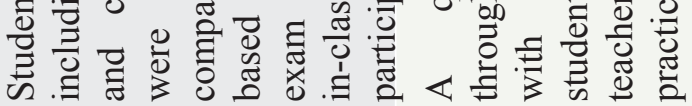

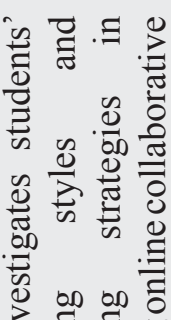

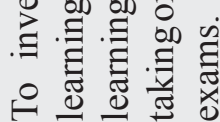

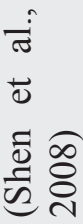

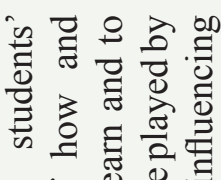

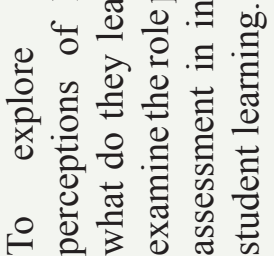

बं

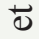

跑令 
Assessment drive students learning, but this drive is not always towards positive direction. Formative assessment is likely to contribute to students' deep approach to learning while summative assessment is likely to contribute to their surface approach. However students tend to swing between different learning approaches in response to various factors. While changing education culture is highly needed in order to accommodate assessment effect on students learning and direct it to enhance deeper approach to learning, assessment impact on students' learning will continue to be strongly correlated with the magnitude of the severity of consequences associated with its implementation. Unless a full understanding to the effect of various personal and contextual factors on students learning is established, students will continue to swing between different learning approaches. Several of these factors have been partially explored, such as assessment function, clinical supervisors and supervision and constructive alignment. There are many others that have yet to be explored in connection to students' perception of their assessment and the adopted learning approaches. This exploration should aim to have a clear guide on how to adopt a customized assessment to education culture, environment and recourses that can positively influence students' learning.

\section{References}

AL KADRI, H. M., AL-MOAMARY, M. S., AL TAKRONI, H., ROBERTS, C. \& VAN DER VLEUTEN, C. (2011a) Self-Assessment and Students' Study Strategies in a Community of Clinical Practice: A Qualitative Study. Riyadh, Saudi Arabia, KSAU-HS, COM (Unpublished Data).

AL KADRI, H. M., AL-MOAMARY, M. S., ELZUBAIR, M., MAGZOUB, M. E., ALMUTAIRI, A., ROBERTS, C. \& VAN DER VLEUTEN, C. (2011b) Exploring factors affecting undergraduate medical students' study strategies in the clinical years: a qualitative study. Adv Health Sci Educ Theory Pract. DOI. 10.1007/s10459-010-9271-2.

AL KADRI, H. M., AL-MOAMARY, M. S. \& VAN DER VLEUTEN, C. (2009) Students' and teachers' perceptions of clinical assessment program: A qualitative study in a PBL curriculum. BMC Res Notes, 2, 263.

BAETEN, M., DOCHY, F. \& STRUYVEN, K. (2008) Students' approaches to learning and assessment preferences in a portfolio-based learning environment. Instructional Science, 36, 359-374.

BIGGS, J. (1993) What do inventories of students' learning processes really measure? A theoretical review and clarification. Br J Educ Psychol, 63, 3-19. 
BIGGS, J. (1999) Teaching for Quality Learning at University, Buckingham, SRHE and Open University Press.

BIGGS, J. B. (1970) Faculty pattern in study behaviour. Aust. J. Psychol, 22, 161-174.

BIGGS, J. B. (1976) Dimensions of study behaviour: Another look at a.t.i. Br. J. Educ. Psychol, 46, 6880 .

BLACK, P. \& WILIAM, D. (1998a) Assessment and classroom learning. Assessment in Education, 5, 7-74.

BLACK, P. \& WILIAM, D. (1998b) Inside the black box: Raising standards through classroom assessment. Phi Delta Kappan, 80, 139-149.

BOUD, D. (1995) Enhancing learning through self-assessment. Kogan Page. London.

BROADBENT, D. E. (1966) The well-ordered mind. Am. Educ. Res. J, 3 281-295.

BROEKKAMP HEIN, BERNADETTE HAM \& HOUT-WOLTERS, V. (2007) Students' Adaptation of Study Strategies When Preparing for Classroom Tests. Educ Psychol Rev, 19, 401-428.

CILLIERS, F. J., SCHUWIRTH, L. W., ADENDORFF, H. J., HERMAN, N. \& VAN DER VLEUTEN, C. P. (2010) The mechanism of impact of summative assessment on medical students' learning. Adv Health Sci Educ Theory Pract, 15, 695-715.

CILLIERS, F. J., SCHUWIRTH, L. W., HERMAN, N., ADENDORFF, H. J. \& VAN DER VLEUTEN, C. P. (2011) A model of the pre-assessment learning effects of summative assessment in medical education. Adv Health Sci Educ Theory Pract.

COWIE, B. \& BELL, B. (1999) A model of formative assessment in science education. Assessment in Education, 6, 101-116.

DOCHY, F., SEGERS, M., GIJBELS, D. \& STRUYVEN, K. (2006) Assessment engineering Breaking down barriers between teaching and learning, and assessment. IN BOUD, D. J. (Ed.) Rethinking assessment in higher education : learning for the longer term. New York, Routledge.

EDSTROM, K., GAIDI, K. E., HALLSTROM, S. \& KUTTENKEULER, J. (2006) Integrated assessment of disciplinary, personal and interpersonal skills - student perceptions of a novel learning experience. IN RUST, C. (Ed.) Improving Student Learning: Improving Student Learning Through Assessment. Oxford, OCSLD.

ENTWISTLE, N. J. \& ENTWISTLE, D. M. (1970) The relationships between personality, study methods and academic performance. Br. J. Educ. Psychol, 40 132-141.

GIJBELS, D., SEGERS, M. \& STRUYF, E. (2008) Constructivist learning environments and the (im)possibility to change students' perceptions of assessment demands and approaches to learning. Instructional Science, 36, 431-443.

KADRI, H. M., AL-MOAMARY, M. S., MAGZOUB, M. E., ROBERTS, C. \& VleuTEN, C. P. M. (2011) Students' perceptions of the impact of assessment on approaches to learning: a comparison between two medical schools with similar curricula. Int J Med Educ, 2, 44-52.

KLENOWSKI, V. (1996) Connecting assessment and learning. British Educational Research Association Annual Conference. Lancaster University.

KNIGHT, P. T. (2001) A briefing on key concepts: formative and summative, criterion and normreferenced assessment. LTSN Higher Education Academy Generic Centre Assessment Series. New York. $\mathrm{KOH}$, L. C. (2008) Refocusing formative feedback to enhance learning in pre-registration nurse education. Nurse Education in Practice, 8, 223-230. 
LEUNG, S. F., MOK, E. \& WONG, D. (2008) The impact of assessment methods on the learning of nursing students. Nurse Education Today 28, 711-719.

LUWEL, K., LEMAIRE, P. \& VERSCHAFFEL, L. (2005) Children's strategies in numerosity judgment. Cognitive Development, 20, , 448-471.

MARTON, F. \& SALJO, R. (1976) On qualitative differences in learning: I- Outcome and process. British Journal of Educational Psychology Review, 46, 4-11.

MARTON, F. \& SALJO, R. (1997) Approaches to learning. IN MARTON, F., HOUNSELL, D. J. \& ENTWISTLE, N. J. (Eds.) The Experience of Learning. 2nd ed. Edinburgh, UK, Scottish Academic.

MATTICK, K. \& KNIGHT, L. (2007) High-quality learning: harder to achieve than we think? Med Educ, 41, 638-44.

MATTICK, K. \& KNIGHT, L. (2009) The importance of vocational and social aspects of approaches to learning for medical students. Adv Health Sci Educ Theory Pract, 14, 629-44.

MCKEACHIE, W. J. (1990) Research on college teaching: The historical background. J. Educ. Psychol, $82,189-200$.

NEWBLE, D. \& JAEGER, K. (1983) The effect of assessment and examination on the learning of medical students. Med Educ, 17, 165-171.

NEWBLE, D. I. (1988) Eight years' experience with a structured clinical examination. Med Educ, 22 , 200-4.

NIJHUIS, J., SEGERS, M. \& GIJSELAERS, W. (2005) Influence of redesigning a learning environment on student perceptions and learning strategies. Learning Environments Research, 8 67-93.

NORMAN, G., NEVILLE, A., BLAKE, J. M. \& MUELLER, B. (2010) Assessment steers learning down the right road: impact of progress testing on licensing examination performance. Med Teach, 32, 496-9.

PRESCOTT, L. E., NORCINI, J. J., MCKINLAY, P. \& RENNIE, J. S. (2002) Facing the challenges of competency-based assessment of postgraduate dental training: Longitudinal Evaluation of Performance (LEP). Med Educ, 36, 92-7.

RAMSDEN, P. (1984) The context of learning. IN MARTON, F., HOUNSELL, D. \& ENTWISTLE, N. (Eds.) The experience of learning. Edinburgh, Scotland, Scottish Academic Press.

REAY, D. \& WILIAM, D. (1999) 'I'll be a nothing': structure, agency and the construction of identity through assessment. British Educational Research Journal, 25, 345-354.

RUSHTON, A. (2005) Formative assessment: a key to deep learning? Med Teach, 27, 509-513.

SANTY, J. \& MACKINTOSH, C. (2000) Assessment and learning in post-registration nurse education. Nurs Stand, 14, 38-41.

SCHMECK, R., RIBICH, F. \& RAMANAIAH, N. (1977) The development of a self-report inventory for assessing individual differences in learning processes. Appl. Psychol. Meas, 1, 413-431.

SCHUNN, C. D. \& REDER, L. M. (1998) Strategy adaptively and individual differences. IN MEDIN, D. L. (Ed.) The psychology of learning and motivation. New York, Academic.

SCHUWIRTH, L. \& VAN DER VLEUTEN, C. (2004) Merging views on assessment. Med Educ, 38, 1208-10.

SCHUWIRTH, L. W. \& VAN DER VLEUTEN, C. P. (2011) Programmatic assessment: From assessment of learning to assessment for learning. Med Teach, 33, 478-85.

SCOULLER, K. (1996) July Influence of assessment method on students' learning approaches, 
perceptions and preferences: The assignment essay versus the short answer examination. HERDSA. Perth, Australia.

SEGERS, M., MARTENSA, R. \& BOSSCHE, P. (2008) Understanding how a case-based assessment instrument influences student teachers' learning approaches. Teaching and Teacher Education, 24, 1751-1764.

SEGERS, M., NIJHUIS, J. \& GIJSELAERS, W. (2006) Redesigning a learning and assessment environment: The influence on students' perceptions of assessment demands and their learning strategies. Studies in Educational Evaluation, 32, 223-242.

SHEN, J., HILTZ, S. R. \& BIEBER, M. (2008) Learning Strategies in Online Collaborative Examinations. IEEE TRANSACTIONS ON PROFESSIONAL COMMUNICATION, 51, NO. 1.

SLUIJSMANS, D. M. A., BRAND-GRUWEL, S. \& VAN MERRIËNBOER, J. (2002) Peer assessment training in teacher education. Assessment and Evaluation in Higher Education, 27, 443-454.

STRUYVEN, K., DOCHY, F., JANSSENS, S., SCHELFHOUT, W. \& GIELEN, S. (2006) On the dynamics of students' approaches to learning: the effects of the learning/teaching environment. Learning and Instruction, 16, 279-294.

TANG, C. (1994) Effects of modes of assessment on students' preparation strategies. IN GIBBS, G. (Ed.) Improving student learning: Theory and practice. Oxford, Oxford Centre for Staff Development. TILLEMA, H. (2001) Portfolios as developmental assessment tools. International Journal of Training and Development, 5, 126-135.

TIWARI, A., LAM, D., YUEN, K. H., CHAN, R., FUNG, T. \& CHAN, S. (2005) Student learning in clinical nursing education: perceptions of the relationship between assessment and learning. Nurse Educ Today, 25, 299-308.

TORRANCE, H. \& PRAYOR, J. (1998) Investigating Formative Assessment: teaching, Learning and Assessment in the Classroom. Open University Press. Buckingham.

VAN DER VLEUTEN, C. P. \& SCHUWIRTH, L. W. (2005) Assessing professional competence: from methods to programmes. Med Educ, 39, 309-17.

VERMUNT, J. D. (1996) Metacognitive, cognitive and affective aspects of learning styles and strategies: A phenomenographic analysis. Higher Education, 31, 25-50.

VERMUNT, J. D. (1998) The regulation of constructive learning processes. Br. J. Educ. Psychol, 68, 149-171. 

Chapter 7

\section{Discussion}





\section{Introduction and research questions}

The purpose of this thesis is to explore 1) the assessment factors that contribute to students' study strategies in their clinical training and 2) if these factors can be used to drive students' learning favorably, i.e. to drive students' learning towards a deep rather than superficial approach to learning.

In this thesis, King Saud bin Abdulaziz University for Health Sciences, College of Medicine (KSAU-HS, COM) was taken as an example of a college that implemented a mainly summative assessment program, while Sydney Medical School (SMS) was taken as an example of a college that implemented less heavy summative assessment and more formative assessment. The performed work was exploratory rather than definitive: its main goal was to guide development of further enquiries and reach a deeper understanding of how students responded to their assessment and how they would study based on this response.

We aimed to understand how students reacted to summative or formative assessment within the clinical setup and to explore factors that directed students towards deeplearning approaches including knowledge integration, writing summaries, self-testing, clinical problem solving and safe patient management (Groves, 2005, McParland et al., 2004, Newble and Entwistle, 1986).

In the research, we carried out several manipulations and assessed students' perception of both summative and formative assessments in several contexts and conditions. These manipulations were done to assist answering the following research questions:

1- What is the relationship between a learning environment, assessment demands as perceived by students and students' approaches to learning in a community of clinical practice? (Chapter 2). 
2- What are the different assessment factors, including feedback, which contributes to students' study strategies in the clinical context or a community of clinical practice?, and can these factors be classified as promoting one of the different known study strategies? (Chapters 3,4 and 5).

3- Where our results do stand within the available research on assessment effects on students learning in general and within a clinical context specifically? (Chapter 6).

In this chapter, we will start by reporting the main findings and the theoretical conclusions of the studies performed. Some limitations of the research will be described next, followed by suggestions for future research and implications on practice. The manuscripts that are forming this thesis include a literature review on assessment effects on students learning approaches. In this review, we have discussed our results within the bulk of the published literatures on the topic. Therefore, we did not discuss the results of each manuscript separately rather; we opted to keep that when we present the literature review and the general conclusion of the thesis.

\section{Main findings and conclusions}

\section{Q1: What is the relationship between a learning environment, assessment demands} as perceived by students and students' approaches to learning in the community of clinical practi ce?

In chapter 2, we have addressed the above question concerning the different factors contributing to students' study strategies in a clinical setup.

In this research, we evaluated students' assessment actions and interactions in the community of clinical practice and the effect of such actions on students' study strategies. 
Our findings revealed that students' ongoing activities and their involvement in the community of clinical practice manipulate their learning strategies. Students found to be very strategic or adaptive when they learn and they manipulated their studying based on what environment required them to do (Groves, 2005, Ramsden and Entwistle, 1981, Schmidt et al., 2010, Seabrook, 2003, Lave and Chaiklin, 1993). We found that the factors affecting students' study strategies are students' clinical training and its resulting stress, clinical supervisors and supervision and the implemented assessment. Depending on whether these factors have a negative or positive impact on a learning environment, students' study strategies become increasingly surface oriented, swing between surface, deep, effort and achievement motivation or reflect a deep learning strategy.

For example, a teacher's personality, availability, training and effectiveness as a role model may have a positive or negative impact on students' approaches to learning. We found that depends on how teachers act and interact with their students their potential influence on students' learning may carry a constructive or a destructive effect (Kennedy et al., 2009, Gray et al., 2008).

Upon analyzing the three main factors identified as major contributors to students' study strategies in a clinical setup (clinical supervisors and supervision, stress and anxiety and assessment), we notice that these factors are strongly linked to students' assessment. Assessment related stress during students' training is connected largely to the clinical environment. In this environment, the clinical supervisors are playing a major role in students' assessment implementation and in alleviating or aggravating this stress (figure 1).

In chapters' 3, 4 and 5 we have addressed the second research question about assessment factors which contribute to students' different study strategies in a clinical setup. We will summarize the results of these researches and present it one by one. 


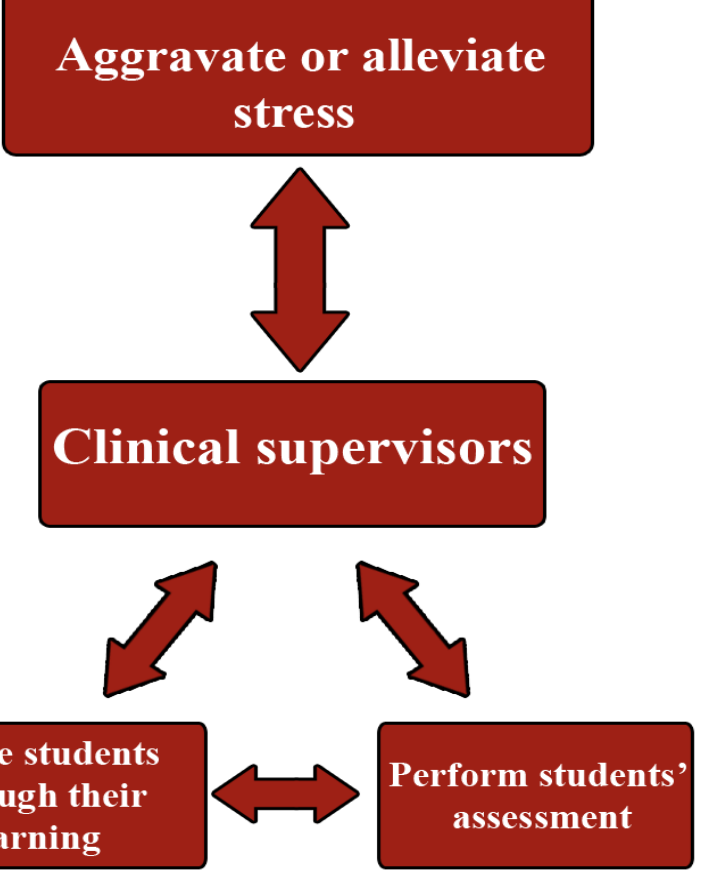

Figure 1: The inter-relation between factors contributing to students' study strategies in a clinical context

Q2: What are the different assessment factors, including feedback, that contribute to students' study strategies in the clinical context or "community of clinical practice", and can these factors be classified as promoting one of the different known study strategies?

The answer of this question was coverd in three different researches.

In chapter 3, we assessed students' and their teachers' perception of the clinical assessment program. The results of this research have confirmed that assessment affects students' perception of learning and how they learn. These effects are not 
uniformly positive. Assessment may lead students to a deep approach to learning "analytical thinking, objectives directed studying, relating patients' conditions to patients' management and others". Assessment may also lead to a superficial approach to learning, "patchy and superficial reading and memorization without understanding or without linking between the clinical and theoretical knowledge".

We found that the predominantly summative assessment program with minimal engagement in formative assessment has offered little inducement to engage in deep learning. Linking students' accumulative GPAs to their future work opportunities cause a lot of stress. An education culture that values performance on the basis of students' hunted marks adds more stress to the students and results in a negative influence on their learning approaches. This stress leads to students' patchy and fragmented reading and transforms the students into mark seekers. On the contrary, students' clear preference of formative assessment may foster a deeper approach to learning. Formative assessment allows students to identify their weaknesses and formulate an improvement plan. It assists learning objectives directed studying and facilitates understanding of the problems of the patients. It also helps in obtaining relevant information on patients' management and the utilization of adequate and correct learning resources.

We realized in this research that teachers have a simple and rather conventional idea of stimulating students' learning. They prefer summative assessment to formative as a factor that leads to better studying and a deeper approach to learning. They believe that the only and first priority in designing an assessment program is to serve the purpose of students' accountability, ranking and certifying competence, while they deny its remarkable side effect on students' learning. It appears that teachers' education culture backgrounds, favoring summative over formative assessment, has an influence on how they perceive an assessment program and how they respond to the implemented 


\section{Discussion}

assessment. Part of this influence might be eliminated and corrected through the implementation of faculty improvement programs. However, major correction can only happen when we manage to change the education culture, a difficult task to be achieved.

In chapter 4, we explored the different cultural and contextual factors that affected students' perception of their assessment program and their approaches to learning. In this research, we tried to generate a richer understanding of the full range of opinions and experiences of students when they are exposed to the implemented assessment. We described the interactive relationship among student factors, teaching context, the on-going approaches to a particular task and students' learning outcomes. The findings have indicated that the important factors affecting students' perception of their assessment include the function of assessment used (summative or formative), students' perception of curriculum objectives and learning outcome, and the presence and nature of authentic clinical assessment with the involvement of clinical supervisors. Summative assessment results in the enhancement of students' motivation to learn at the cost of both a superficial approach and an effort and achievement-motivation approach to learning. However, formative assessment implementation with its feedback results in a tendency toward a deep approach to learning. These effects are not similar among students. The observed variations in students' study strategies are influenced by assessment function, students' cultural backgrounds and personal values and preferences. Students' study approaches vary according to the implemented assessment function whether summative or formative. The influence of these different assessment functions on students' future opportunities plays a role in how students learn. Whenever students' future job or study opportunities depend on their grades and accumulative GPA, summative assessment plays a role in students' stress, patchy reading and superficial or effort and achievement motivation study strategies. On the other hand, when mark or accumulative GPA doesn't have a significant influence on students' future study or job opportunities, students favor 
summative assessment and wouldn't even mind the stress resulting from it. They even consider this stress as a stimulus for them to better learning.

In addition to the previous contextual factors affecting students' perception of their assessment and their approaches to learning there are cultural differences and cultural backgrounds. Students from different cultures have different perceptions and attitudes towards different assessment functions. Students from a multi-cultural background, such as the students' culture in Sydney, are more accommodating to stress and supervisors' feedback, even if it is negative. On the other hand, students from ethnically homogenous population, such as the Saudi culture, are less accommodating to stress and negative feedback. For such students who are heavily occupied with their personal and environmental values, formative assessment represents an important positive drive to their learning only if it is used in the right circumstances and with correct preimplementation orientation. Overall, there is a fine interplay between characteristics of the learners, their perception of learning, cultural background, motivation and characteristics of the assessment whether summative or formative (figure 2).

It appears that a cultural sensitive balance, between the implemented summative and formative assessments, is needed to drive students' learning towards more favorable and deeper approach to learning. Every institution needs to assess its own students'

perception of their assessment and decide how much formative and summative assessment they need to implement to drive students learning towards deep rather than surface approach to learning.

In chapter 5, we tried to reach a richer understanding of the relationship between students' perceptions and practice of self-assessment (as an example of an assessment that is used mainly for formative purposes) and students' study strategies within a "community of clinical practice". 


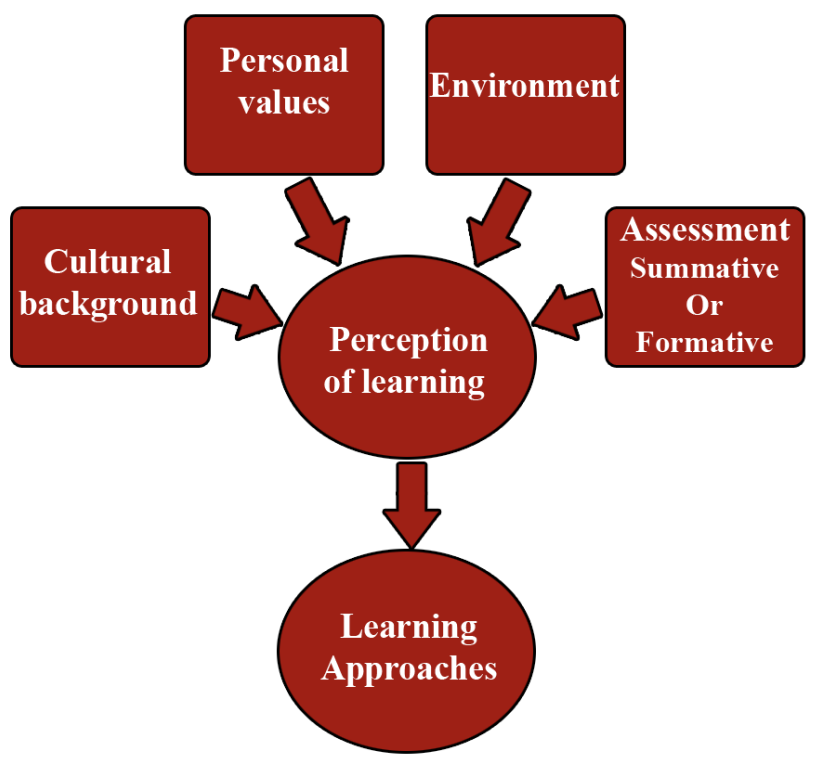

Figure 2: The interplay between characteristics of learners, their environment, culture, implemented assessment and approaches to learning

It was found that several factors contributed to students' perception of self-assessment and their study strategies. Students favor training on how to practice self-assessment and benefit from feedback. However, this training doesn't change students' strategic practice of self-assessment or their tendency to overestimate their performance. It doesn't also contribute to their perception of patient care or their ability to directly encounter patients and maintain patients' safety. Regardless of the strategy used for self-assessment, the practice is influenced by students' attempts to manipulate their supervisors' summative assessment to achieve higher marks or simply to leave a good impression. This results in an achievement-motivation study strategy and turns the students into grade-seekers. Students value formative feedback and agree that it contributes to a deeper approach to learning. Similar to our previous research, it was confirmed that negative feedback contributed to negative feelings and patchy reading. 
Students consider negative feedback insulting and undermining. The embarrassment resulting from negative feedback lead to unwanted learning results including superficial and achievement motivation study strategies.

\section{Q3: Where our results do stand within the available research on assessment effects on students learning in general and within a clinical context specifically?}

In chapter 6, we explored the published literatures, including our work, which have discussed assessment effects on students learning approaches. The majority of identified literatures were discussing general assessment factors contributing to students' approaches to learning. Some literatures have also focused on various aspects of either summative assessment or formative assessment or were in the form of literature reviews.

A small number of literatures have addressed the swinging study strategies practiced by the students between surface and deep approaches to learning e.g. (Al Kadri et al., 2011b, Mattick and Knight, 2007, Mattick and Knight, 2009, Newble and Jaeger, 1983).

Moreover, there were scarce literatures that assessed circumstances or conditions under which students reacted to their assessment and changed their study approach whether towards deep or surface approach to learning e.g. (Kadri et al., 2011, Al Kadri et al., 2011b, Al Kadri et al., 2009). Furthermore, there were even more scarce literatures that have discussed the role of assessment in driving students' learning in a clinical teaching context (Al Kadri et al., 2011a, Al Kadri et al., 2011b, Al Kadri et al., 2009, Kadri et al., 2011). It appears from this review that our thesis work was of the rare literatures that have addressed the effect of assessment on students' learning in a clinical context.

In this review, the most prominent identified theme was assessment function characterized in summative and formative assessment and general effect of 


\section{Discussion}

assessment on students learning approaches. The reviewed literatures have pointed clearly to the complexity of the relationship between learning environment, students' perceptions of assessment demands, and students' approaches to learning. Many extrinsic and intrinsic factors were theoretically proposed to mediate students' approaches to learning in response to their assessment. However, few of these factors were researched in the published literature.

Assessment was found effective in driving students' learning, but this drive is not always towards positive direction. Formative assessment is likely to contribute to students' deep approach to learning while summative assessment is likely to contribute to their surface approach. However some literatures have shown that students tend to swing between different learning approaches in response to various contextual and personal factors.

In general when students plan to learn and understand, they tend to use a deep approach to learning, but when they prepare for their exams, regardless their perception of learning approaches, they are likely to use less of deep approach to learning and more of surface approach to learning (Tang, 1994). This is found to be true even with the introduction of new modes of assessment (Struyven et al., 2006) that requires more deep thinking and analytic approach. It is also true even with the change of learning environment through education, explanation and orientation on the benefits of certain assessment tool or certain learning approach (Al Kadri et al., 2011a, Shen et al., 2008). To maximize the positive effect of assessment on students' learning, particularly formative assessment, it seems that we need to go beyond teaching and orientation into changing education culture. Meanwhile and till we succeed to achieve a favorable culture change, assessment impact on students' learning will continue to be strongly correlated with the magnitude of the severity of consequences associated with its implementation. 


\section{General conclusion}

We can conclude from this series of researches that several intrinsic and extrinsic assessment factors have been found to affect students' learning and learning approaches. Many of these factors were theoretically proposed by Broekkamp et. al. (Broekkamp Hein et al., 2007) but few of them have been already researched. These factors are either assessment derived with particular assessment functions whether summative or formative or factors that are related to assessment indirectly such as curriculum objectives, clinical supervision and supervisors, assessment environment and assessment culture.

Assessment factors are perceived by the students differently and result in inducement of different learning approaches. For some students, summative assessment induces a superficial approach to learning, while for others it results in a deeper approach to learning, the same is true for formative assessment. The different views in the published literatures concerning both summative and formative assessment such as (Rushton, 2005, Tillema, 2001, Baeten et al., 2008, Koh, 2008, Black and Wiliam, 1998, Sluijsmans et al., 2002, Cilliers et al., 2010, Cilliers et al., 2011, Leung et al., 2008) are possibly related to the presence of other mediators that are affecting students' learning approaches and perceptions of their assessment. Part of these mediating factors are related to the implemented health care setup that appreciate students performance based on their grades and summative achievements rather than the quality of students' learning outcomes (Harlen, 2005). Future job opportunities and availabilities could play also the role of mediator affecting students' perceptions of their assessment and their learning approaches. For example where future job offer or post graduate studies are related to students accumulated GPA, there will be more tendencies to surface or achievement motivating approaches to learning. Moreover, education culture background, students' age and gender may play a role of mediators affecting students' 


\section{Discussion}

perception of their assessment and their learning approaches. We believe that more in depth researches are needed to cover this area.

Balancing between the different effects of assessment factors on students' study strategies is needed. However, it will be difficult to find a unified balanced formula between summative and formative assessment that suits all circumstances, all students, all cultures and all institutions; cases have to be individualized. Work needs to be done within each institution aiming to explore the correct balance between formative and summative assessment that fit its culture and environment. Each institution should study and explore students and teachers' cultural backgrounds, beliefs and perceptions, available resources, health education system and others. Based on this information, educators may decide the extent of the use of either summative or formative assessment that suits a particular institution during a specific period of time. This balance should be enough to promote students' learning without compromising the other goals of assessment such as assessing students' accountability, ranking among peers and guaranteeing the achievement of the required pre-planned certifying competences. For example for the studied institution in Saudi Arabia, more focus on formative assessment at the expense of summative assessment with more involvement of the students in patients' care and direct patients contact may lead to better approach to learning. Attempting to eliminate the negative effect of the summative assessment on students' future opportunities and job availabilities could play a strong positive influence on students approach to learning, improve on their self esteem and confidence and alleviate the stress that might result of summative assessment.

On the other hand, although we found that a teacher's personality, availability, training and effectiveness as a role model carry a positive impact on students' approaches to learning; teachers tend to have a simple rather than conventional idea of stimulating students' learning. They prioritize assessment as a method of assessment of learning 
rather than a method of assessment for learning (Dylan et al., 2004, Schuwirth and Van der Vleuten, 2011). Teachers' perception of assessment and learning sometimes contradict the students' views and negatively influence the assessment power as a drive for students' learning. It was appreciated in this work as many others (Branch et al., 2009, Steinert et al., 2006, Stern et al., 2000) that faculty development and teaching and orientation on assessment role in students' learning may partially close the gap between students' and teachers' perceptions of the implemented assessment.

However, this effort is not enough on its own, faculty development is been repeatedly reported as a difficult task and is not always successful in the absence of adequate circumstances and requirements (O'Sullivan and Irby, Steinert and Mann, 2006). Better success in eliminating the presence of such gap can be achieved through a long term plan aiming to change education culture (Steinert and Mann, 2006).

\section{Suggestions for further research}

The discussed researches have evaluated students' perception of their assessment and the resulting learning strategies. Further work on the effect of assessment on students' learning outcome, patient care and patients' satisfaction is needed.

In our first research (chapter 2), we have evaluated only the first two levels of Kirkpatrick's learning and training evaluation (students' reaction and their learning in the clinical context). To evaluate the third and fourth levels (Kirkpatrick, 1994, Phillips, 1996), further research is needed. This will enable us to understand the continuous interaction among students, teachers, educational environment and patient care and satisfaction.

We have explored the effects of assessment on students' learning strategies in all clinical contexts. Further research is needed to explore learning strategies in specific clinical specialties that carry special characteristics, such as the intensive care units, palliative 


\section{Discussion}

care units and obstetrics care units. Moreover, further research to assess these effects and the direction in other health education setups is needed.

Although we were unique in exploring the effects and directions of different assessment factors contributing to students' study strategies within a clinical context "deep, superficial, effort and achievement motivation strategies", we did not assess all the intrinsic and extrinsic factors suggested by Broekkamp to contribute to this effect (Broekkamp Hein et al., 2007). Further researches to fully assess these factors effects and directions on students learning are needed. In fact, we have shown through the performed literature review that most of the claims of the benefits of formative assessment in enhancing a deep approach to learning were theoretical. Further research to test this work through putting the existing theories into practice is recommended.

Finally, we recommend a research that assesses the differences between male and female students' perception of their assessment and the effects of these differences on students' approaches to learning. Moreover, further researches that focus more on the various personal, contextual and cultural factors affecting students' perception of their assessment and the resulting study strategies are needed.

\section{Limitations}

We have identified in this work several limitations that need to be noted and discussed. First: Our researches were mainly based on two medical colleges, which may limit the possibility of generalizing our findings. However, different data collection methods were used to ensure that all participants were exposed to similar challenges and were given similar chances to express their feelings and perceptions within the studied setup. We used several tools to improve on the work rigors such as data triangulation and peers debriefing. Overall, we aimed to give a clear unbiased example of students' learning behavior in the studied "communities of clinical practice". We have evaluated the assessment program and included the main stakeholders taking into consideration 
the instructional design and the used curriculum while reflecting on other published researches on students' assessment. Through the implemented methodologies, we believe that we worked to achieve transferability.

Second: Our studies design was limited to students' and teachers' views and experiences and did not include the relationship between learning strategies and students' learning outcomes. In other words, we focused on pre-assessment effects on students' learning (Rushton, 2005). A relatively recent study from the Netherlands found no significant relationship between learning strategies and clinical performance (van Lohuizen et al., 2009) or post assessment effect, but we acknowledge that this is an important area for further work.

Third: The population at KSAU-HS, COM consisted entirely of male students. In the Saudi education culture male and female are taught the same curriculum but separately. We are not aware of previous comparisons between male and female students regarding their perception of exams, but a gender effect cannot be excluded.

Fourth: A limitation related to our third research presented in chapter four lies in the difficulty of comparing two very different groups of students. Additional cultural factors may have contributed to differences in students' study strategies, for example the differences between the multicultural society of Sydney and the ethnically homogenous Saudi student population at KSAU-HS, COM. Furthermore, despite the fact that similar curricula were implemented in both schools, we could not deny the possibility of curricular differences.

\section{Implications for practice}

Our study findings provide some insights into the influence of assessment related contextual factors on students approach to learning. From these findings, we would suggest the following additional implications. 
1- Any institution planning to implement an educational curriculum that is designed and implemented in a totally different setup should review the similarity of various issues that may play a role in the success or failure of this implementation. Within the area where the curriculum is originally designed for and the adopting implementation areas, there should be similarity in the health care programs implemented, level of faculty education experiences (O'Sullivan and Irby), cultural background (Tervalon, 2003, Seabrook, 2003), post graduate studies requirements and new graduates job opportunities. The presence of considerable cultural, environmental or resources differences between the two implementation areas may lead to inappropriate and disappointing outcomes even for the best designed curriculums. Blind borrowing of educational curriculums should be discouraged rather conscious adoption of curriculum with preparation that includes close liaison between the curriculum developers of the resources, careful and systematic planning and the presence of educator and a supporting administrative staff who ensure the appropriate use of the adopted curriculum (Issenberg et al., 2003).

2- Conflict between students and teachers beliefs, their education culture and the implemented assessment program may stand as a major obstacle behind curriculum implementation failure (Seabrook, 2003). Culture is found to be affecting students' perceptions of the implemented assessment. The culture differences may lead to differences in how students and their teachers perceive assessment (Tervalon, 2003). Therefore, culture change that aims to accommodate both summative and formative assessment should be the target of health education planners. Such a change will enable easier implementation of different assessment tools utilizing different assessment function while guaranteeing their drives to better learning approaches (Christianson et al., 2007). As culture change is known to be hard to achieve, 
such effort is recommended as early as possible during students' education years. This effort should be directed towards buffering the negative effect of the consequences of summative assessment (Cilliers et al., 2010, Cilliers et al., 2011) with more power to formative assessment. Moreover, it needs buffering the negative effect of both summative and formative assessment (Teelucksingh et al., 2001, Thistlethwaite, 2006) with better understanding to their effect on students' learning. Such a gradual conscious move that is directed to increase awareness and improve on accommodation of different assessment functions may lead on the long run to change in the beliefs and understanding of both students and teachers, decrease the gap in their perceptions of assessment function and result in students' better approach to learning.

3- Both health care system and health education planning that are focusing on patients' outcome should be planned in parallel (Shackelton et al., 2009). The two systems planning should not be separated at any governing area as they are strongly related and do influence each other. A culture sensitive and needs fulfilling health care system and medical education assessment program should be studied and implemented as one program that aims to achieve the health care outcome as planned by the government.

4- Although this work has explored the effect of assessment on students' approaches to learning. Our findings have suggested that this area is very complicated and significantly under-researched. We consider this thesis as a modest step that explores this important aspect of students learning. Moreover, we call through this work for a move of assessment research to cover this very important subject on the effect of assessment on students' learning. 


\section{References}

AL KADRI, H. M., AL-MOAMARY, M. S., AL TAKRONI, H., ROBERTS, C. \& VAN DER VLEUTEN, C. (2011a) Self-Assessment and Students' Study Strategies in a Community of Clinical Practice: A Qualitative Study. Riyadh, Saudi Arabia, KSAU-HS, COM (Unpublished Data).

AL KADRI, H. M., AL-MOAMARY, M. S., ElZUBAIR, M., MAGZOUB, M. E., ALMUTAIRI, A., ROBERTS, C. \& VAN DER VLEUTEN, C. (2011b) Exploring factors affecting undergraduate medical students' study strategies in the clinical years: a qualitative study. Adv Health Sci Educ Theory Pract. DOI. 10.1007/s10459-010-9271-2.

AL KADRI, H. M., AL-MOAMARY, M. S. \& VAN DER VLEUTEN, C. (2009) Students' and teachers' perceptions of clinical assessment program: A qualitative study in a PBL curriculum. BMC Res Notes, 2, 263.

BAETEN, M., DOCHY, F. \& STRUYVEN, K. (2008) Students' approaches to learning and assessment preferences in a portfolio-based learning environment. Instructional Science, 36, 359-374.

BLACK, P. \& WILIAM, D. (1998) Assessment and classroom learning. Assessment in Education, 5, 7-74.

BRANCH, W. T., JR., FRANKEL, R., GRACEY, C. F., HAIDET, P. M., WEISSMANN, P. F., CANTEY, P., MITCHELL, G. A. \& INUI, T. S. (2009) A good clinician and a caring person: longitudinal faculty development and the enhancement of the human dimensions of care. Acad Med, 84, 117-25.

BROEKKAMP HEIN, BERNADETTE HAM \& HOUT-WOLTERS, V. (2007) Students' Adaptation of Study Strategies When Preparing for Classroom Tests. Educ Psychol Rev, 19, 401-428.

CHRISTIANSON, C. E., MCBRIDE, R. B., VARI, R. C., OLSON, L. \& WILSON, H. D. (2007) From traditional to patient-centered learning: curriculum change as an intervention for changing institutional culture and promoting professionalism in undergraduate medical education. Acad Med, 82, 1079-88.

CILliERS, F. J., SCHUWIRTH, L. W., ADENDORFF, H. J., HERMAN, N. \& VAN DER VLEUTEN, C. P. (2010) The mechanism of impact of summative assessment on medical students' learning. $A d v$ Health Sci Educ Theory Pract, 15, 695-715.

CILLIERS, F. J., SCHUWIRTH, L. W., HERMAN, N., ADENDORFF, H. J. \& VAN DER VLEUTEN, C. P. (2011) A model of the pre-assessment learning effects of summative assessment in medical education. Adv Health Sci Educ Theory Pract.

DYLAN, W., LEE, C., HARRISON, C. \& BLACK, P. (2004) Teachers developing assessment for learning: Impact on student achievement. Assessment in Education: Principles, Policy and Practice, 11, 49-65.

GRAY, C. S., HILDRETH, A. J., FISHER, C., BROWN, A., JONES, A., TURNER, R. \& BOOBIS, L. (2008) Towards a Formative Assessment of Classroom Competencies (FACCs) for postgraduate medical trainees. BMC Med Educ, 8, 61 .

GROVES, M. (2005) Problem-based learning and learning approach: is there a relationship? Adv Health Sci Educ Theory Pract, 10, 315-26.

HARLEN, W. (2005) Teachers' summative practices and assessment for learning tensions and synergies. The Curriculum Journal, 16, 207 - 223.

ISSENBERG, S. B., PRINGLE, S., HARDEN, R. M., KHOGAli, S. \& GORDON, M. S. (2003) Adoption and integration of simulation-based learning technologies into the curriculum of a UK 
Undergraduate Education Programme. Med Educ, 37 Suppl 1, 42-9.

KADRI, H. M., AL-MOAMARY, M. S., MAGZOUB, M. E., ROBERTS, C. \& VLEUTEN, C. P. M. (2011) Students' perceptions of the impact of assessment on approaches to learning: a comparison between two medical schools with similar curricula. Int J Med Educ, 2, 44-52.

KENNEDY, T. J., REGEHR, G., BAKER, G. R. \& LINGARD, L. A. (2009) 'It's a cultural expectation...' The pressure on medical trainees to work independently in clinical practice. Med Educ, 43, 645-53.

KIRKPATRICK, D. L. (1994) Evaluating Training Programs, San Francisco, Berrett-Koehler Publishers, Inc.

$\mathrm{KOH}$, L. C. (2008) Refocusing formative feedback to enhance learning in pre-registration nurse education. Nurse Education in Practice, 8, 223-230.

LAVE, J. \& CHAIKLIN, S. (1993) Understanding Practice: Perspectives on Activity and Context, Cambridge, University of Cambridge Press.

LEUNG, S. F., MOK, E. \& WONG, D. (2008) The impact of assessment methods on the learning of nursing students. Nurse Education Today 28, 711-719.

MATTICK, K. \& KNIGHT, L. (2007) High-quality learning: harder to achieve than we think? Med Educ, 41, 638-44.

MATTICK, K. \& KNIGHT, L. (2009) The importance of vocational and social aspects of approaches to learning for medical students. Adv Health Sci Educ Theory Pract, 14, 629-44.

MCPARLAND, M., NOBLE, L. M. \& LIVINGSTON, G. (2004) The effectiveness of problem-based learning compared to traditional teaching in undergraduate psychiatry. Med Educ, 38, 859-67.

NEWBLE, D. \& ENTWISTLE, N. (1986) Learning styles and approaches: implications for medical education. Med Educ, 3, 162-175.

NEWBLE, D. \& JAEGER, K. (1983) The effect of assessment and examination on the learning of medical students. Medical Education, 17, 165-171.

O'SULLIVAN, P. S. \& IRBY, D. M. Reframing research on faculty development. Acad Med, 86, 421-8. PHILLIPS, J. (1996) How much is the training worth? Training and Development, 50, 20-24.

RAMSDEN, P. \& ENTWISTLE, N. J. (1981) Effects of academic departments on students' approaches to studying. British Journal of Educational Psychology Review, 51, 368-383.

RUSHTON, A. (2005) Formative assessment: a key to deep learning? Medical Teacher, 27, 509-513.

SCHMIDT, H. G., COHEN-SCHOTANUS, J., VAN DER MOLEN, H. T., SPLINTER, T. A. W., BULTE, J. \& HOLDRINET, R. L. (2010) Learning more by ebting taught less: A "time-for-self-study" theory explaining curricular effects on graduation rate and study duration. Higher Education, 60, 287-300.

SCHUWIRTH, L. W. \& VAN DER VLEUTEN, C. P. (2011) Programmatic assessment: From assessment of learning to assessment for learning. Med Teach, 33, 478-85.

SEABROOK, M. A. (2003) Medical teachers' concerns about the clinical teaching context. Med Educ, 37, 213-22.

SHACKELTON, R., LINK, C., MARCEAU, L. \& MCKINLAY, J. (2009) Does the culture of a medical practice affect the clinical management of diabetes by primary care providers? J Health Serv Res Policy, 14, 96-103.

SHEN, J., HILTZ, S. R. \& BIEBER, M. (2008) Learning Strategies in Online Collaborative Examinations. IEEE TRANSACTIONS ON PROFESSIONAL COMMUNICATION, 51, NO. 1.

SLUIJSMANS, D. M. A., BRAND-GRUWEL, S. \& VAN MERRIËNBOER, J. (2002) Peer assessment 
training in teacher education. Assessment and Evaluation in Higher Education, 27, 443-454.

STEINERT, Y., MANN, K., CENTENO, A., DOLMANS, D., SPENCER, J., GELUlA, M. \& PRIDEAUX, D. (2006) A systematic review of faculty development initiatives designed to improve teaching effectiveness in medical education: BEME Guide No. 8. Medical Teacher, 28, 497 - 526.

STEINERT, Y. \& MANN, K. V. (2006) Faculty Development: Principles and Practices. Journal of Veterinary Medical Education, 33, 317-324.

STERN, D. T., WILLIAMS, B. C., GILL, A., GRUPPEN, L. D., WOOLLISCROFT, J. O. \& GRUM, C. M. (2000) Is there a relationship between attending physicians' and residents' teaching skills and students' examination scores? Acad Med, 75, 1144-6.

STRUYVEN, K., DOCHY, F., JANSSENS, S., SCHELFHOUT, W. \& GIELEN, S. (2006) On the dynamics of students' approaches to learning: the effects of the learning/teaching environment. Learning and Instruction, 16, 279-294.

TANG, C. (1994) Effects of modes of assessment on students' preparation strategies. IN GIBBS, G. (Ed.) Improving student learning: Theory and practice. Oxford, Oxford Centre for Staff Development. TEELUCKSINGH, S., ALI, Z., FRASER, H. S., DENBOW, C. E. \& NICHOLSON, G. D. (2001) Fifty years of clinical examinations at the University of the West Indies. West Indian Med J, 50 Suppl 4, 50-2. TERVALON, M. (2003) Components of culture in health for medical students' education. Acad Med, 78, 570-6.

THISTLETHWAITE, J. (2006) More thoughts on 'assessment drives learning'. Med Educ, 40, 1149-50. TILLEMA, H. (2001) Portfolios as developmental assessment tools. International Journal of Training and Development, 5, 126-135.

VAN LOHUIZEN, M. T., KUKS, J. B., VAN HELL, E. A., RAAT, A. N. \& COHEN-SCHOTANUS, J. (2009) Learning strategies during clerkships and their effects on clinical performance. Med Teach, 31, e494-9. 
Chapter 8

\section{Summary}



The purpose of this thesis was to explore 1) assessment factors that contribute to students' study strategies in clinical training and 2) if these factors can be used to drive students' learning in a desirable direction, i.e. towards a deep rather than a superficial approach to learning. We aimed to understand how students reacted to summative or formative assessment in the clinical setting and we explored factors that stimulated students to adopt deep-learning approaches, such as integration of knowledge, writing summaries, self-assessment, clinical problem solving and patient safety. The seven chapters of this dissertation consist of the general introduction, descriptions of the studies that were conducted, the general discussion, the summary and acknowledgements.

Chapter 1 introduces the aim of the dissertation and summarizes theories on assessment and their effect on change in educational practice and assessment practice. We describe the paucity of the published research in the area of the contribution of assessment factors to students study strategies and we present the research questions. The following research questions were addressed: 1) What is the relationship between the learning environment, assessment demands as perceived by students, and students' approaches to learning in the "community of clinical practice"? 2) Which different assessment factors, including feedback, contribute to students' study strategies in the clinical setting and in the "community of clinical practice"? Can these factors be categorized according to which known study strategy they promote? 3) How do our results fit within the available research on the effects of assessment on the learning of students in general and on learning in the clinical setting in particular?

Chapter 2 reports on a study conducted in a Saudi College of Medicine. The aim of this study was to explore the effects of clinical supervision and assessment characteristics on the study strategies used by undergraduate medical students in their clinical rotations. We conducted a qualitative phenomenological study using semi-structured focus group 
interviews with students and individual interviews with teachers and students to explore students' and clinical teachers' perceptions and interpretations of factors influencing students' study strategies. Analysis of the data from the qualitative interviews with 28 students and thirteen clinical supervisors yielded three major themes relating to factors affecting students' study strategies: "clinical supervisors and supervision”, "stress and anxiety" and "assessment". These three themes played a role in students' adoption of different study strategies in the "community of clinical practice". It appeared in this research that teachers played a key role, notably as assessors, clinical supervisors and as a source of stress to students.

Chapter 3 describes the second phenomenographical, qualitative study using focus group interviews with students and individual interviews with teachers. We explored how and why students differed in their approaches to learning, how assessment affected deep learning, and which barriers stood in the way of good assessment and learning in the clinical years of a Problem-Based Learning (PBL) graduate entry medical curriculum. Our findings in this study confirmed that assessment affects students' perceptions of learning as well as the way they learn. These effects are not uniformly positive. According to the students, the predominantly summative assessment programme offered little inducement to engage in deep learning. Students expressed a clear preference for formative assessment, which can foster a deeper approach to learning.

Chapter 4 presents the third study, which investigated students' perceptions of assessment and how these related to their learning styles. We conducted semi-structured qualitative interviews with students. The analysis of the data resulted in three main themes: the function of assessment, learning outcomes and authentic assessment in the clinical environment. In this study we found that cultural differences and emotions may affect students' perceptions of assessment and their learning styles. Moreover, it appeared that a combination of formative and summative assessments based on learning 
objectives is required if assessment is to achieve educational goals. This combination should be designed to take into consideration students' cultural background, values and the education system in which students are learning. An appropriate balance between these factors is expected to motivate students to maintain their focus and attention and to mitigate potentially negative effects of the hidden curriculum.

Chapter 5 presents the fourth study aimed at achieving a clear understanding of the relationship between students' perceptions and practices of self-assessment and the study strategies they use within the "community of clinical practice". In a phenomenological qualitative study, we interviewed three different student cohorts that were exposed to different contextual factors. We explored students' perceptions and interpretations of self-assessment factors, particularly "self-assessment practice" and "feedback from faculty". We explored how these contextual factors influenced students' study strategies. The analysis of the interviews yielded three major themes with respect to how self-assessment factors affected students' study strategies: strategic practice of self-assessment, self-assessment and students' study strategies, and feedback and students' study strategies. The results showed that the practice of selfassessment does not fit well within a context with summative assessment, and that the implementation of self-assessment requires cultural preparation. Moreover, of the two major components of the self-assessment process, feedback appeared to be the most effective way of enhancing students' study strategies. Feedback may be the component of self-assessment that is mainly responsible for positive effects of self-assessment reported in the literature.

Chapter 6 describes a review of the literature on the effects of the educational environment, with a focus on the effects of assessment on students' learning approaches. We searched Pubmed, PsychINFO and Medline without any restrictions regarding type or date of publication. The most prominent theme that was identified in the publications 
that were included in the review was the function of assessment, characterized as summative or formative assessment, and the general effect of assessment on students' learning approaches. The literature review underlined the complexity of the relationship between the learning environment, students' perceptions of assessment demands, and students' approaches to learning. Theoretically, many extrinsic and intrinsic factors are assumed to mediate students' approaches to learning in response to assessment. However, few of these factors were addressed in the studies included in the review. What can be concluded from the review is that formative assessment is likely to promote a deep approach to learning, whereas summative assessment is likely to contribute to a surface approach. However, no final conclusions can be drawn with regard to these effects, and further research is needed to clarify the complex relationship between assessment and students' learning strategies.

Chapter 7 presents the general discussion of the studies in this dissertation focusing on the findings, the limitations of the studies and recommendations made on the basis of the results. We highlight that assessment factors are perceived in different ways by students and can lead to different learning approaches. For some students, for example, summative assessment induces a superficial approach to learning, while for others it results in a deeper approach; the same can be said with regard to formative assessment. The different views voiced in the literature on summative and formative assessment may be due to the presence of other mediating factors affecting students' learning approaches and perceptions of assessment. It seems important to strike a balance between the different effects of assessment factors on students' study strategies. However, it will be difficult to arrive at a single formula for ensuring an effective balance between summative and formative assessment that will suit all circumstances, all students, all cultures and all institutions; it seems more likely that different cases require different combinations of assessment factors. Based on the insights from the studies in this dissertation into the impact of assessment factors on students' study strategies, the 
following recommendations are made: 1) Curricula should be designed and customized to fit with the specific education culture, available facilities, job opportunities and health care system where they are to be implemented. Such customization seems likely to enhance the chances of successful implementation of a curriculum. 2) Substantial efforts should be made to improve the educational culture and reduce the gap between students and teachers' beliefs and perceptions in order to enhance the quality of the learning of students and to stimulate students to adopt a deeper approach to learning. 3) Apart from the insights and recommendations resulting from the results of the studies, we have identified a serious shortage of published studies on the relationship between assessment and study strategies, and we therefore strongly recommend that further studies be conducted in this area. 

Chapter 9

\section{Samenvatting}



Dit proefschrift beschrijft onderzoek naar 1) toetsfactoren die een positieve invloed kunnen hebben op de leerstrategieën die toegepast worden door studenten in de klinische fase en 2) de vraag of er gebruik gemaakt kan worden van deze factoren om het leren van studenten gunstig te beinvloeden, met name door het bevorderen van diepgaand leren in plaats van een oppervlakking leerstijl. Het doel van de onderzoeken was om inzicht te verwerven in hoe studenten reageren op summatieve en formatieve toetsing in het klinisch onderwijs en om te zoeken naar factoren die diepgaand leren bevorderden, zoals integratie van kennis, het schrijven van samenvattingen, zelfbeoordeling, klinisch redeneren en patiëntveiligheid. De zeven hoofdstukken in dit proefschrift omvatten de algemene inleiding, beschrijvingen van de verschillende onderzoeken, de algemene discussie, de samenvatting, en een dankwoord.

Hoofdstuk 1 beschrijft het doel van dit proefschrift en geeft een samenvatting van verschillende theorieën over toetsing en hun rol ten aanzien van veranderingen in de onderwijspraktijk en de toepassing van toetsing. Opgemerkt wordt dat er zeer weining literatuur bestaat over de relatie tussen toetsfactoren en de leerstrategieën van studenten. De onderzoeken in dit proefschrift betroffen de volgende onderzoeksvragen: 1) Wat is de relatie tussen de mening van studenten over de leeromgeving en de toetsingseisen enerzijds en de manier waarop studenten leren in de "community of practice" in de kliniek anderzijds? 2) Welke verschillende toetsingsfactoren, waaronder feedback, hebben invloed op de leerstrategieën die studenten hanteren in de klinische werkplaats of in de "community of practice" in de kliniek? Kunnen deze factoren geklassificeerd worden op basis van hun specifieke invloed op een van de bekende leerstrategieën? 3 ) Hoe passen de resultaten van dit proefschrift in het huidige onderzoek naar de effecten van toetsing op het leren van studenten in het algemeen en het leren in de klinische praktijk in het bijzonder?

Hoofdstuk 2 beschrijft een onderzoek bij een geneeskundeopleiding in Saudi Arabië naar de effecten van klinische supervisie en toetsingskenmerken op de leerstrategieën 
van studenten tijdens de coschappen. Het betrof een kwalitatief fenomenologisch onderzoek met behulp van semigestructureerde focusgroepen bestaand uit studenten en individuele interviews met docenten en studenten met als doel inzicht te verwerven in de ideeën van studenten en klinisch docenten over factoren die de leerstrategieëen van studenten beïnvloeden en hoe studenten en docenten deze factoren interpreteren. Uit de analyse van de kwalitatieve interviews met 28 studenten en dertien klinisch docenten kwamen drie hoofdthema's naar voren met betrekking tot factoren die de leerstrategieën van studenten beïnvloeden: "klinisch docenten en supervisie", "spanning en onzekerheid" en "toetsing". De drie thema's waren van invloed op de leerstrategieën die de studenten toepasten in de "community of practice" in de klinische praktijk". Docenten bleken een centrale rol te spelen, met name als beoordelaars, klinisch begeleiders en als een bron van spanning voor de studenten.

Hoofdstuk 3 beschrijft het tweede fenomenografische, kwalitatieve onderzoek met behulp van focusgroepen met studenten en individuele interviews met docenten. Onderzocht werd hoe en waarom studenten verschillende leerstrategieën toepasten, hoe toetsing van invloed was op diepgaand leren en welke belemmeringen er waren voor goede toetsing en goed leren in de klinische fase van een probleemgestuurd medisch curriculum voor studenten die al een studie hadden afgerond. De resultaten van het onderzoek bevestigden dat toetsing de mening van studenten over leren en de manier waarop zij leren beïnvloedt. Deze invloed is niet altijd positief. Volgens de studenten biedt het overwegend summatieve toetsprogramma weinig stimulansen voor diepgaand leren. De studenten spraken een duidelijke voorkeur uit voor formatieve toetsing, die wel tot dieper leren kan leiden.

Hoofdstuk 4 beschrijft het derde onderzoek naar de mening van studenten over toetsing en de daaruit voortvloeiende leerstijlen. Uit de analyse van semigestructureerde kwalitatieve interviews kwamen drie hoofdthema's naar voren. Deze betroffen de 
functie van toetsing, leerdoelen en authentieke toetsing in de kliniek. Uit het onderzoek bleek dat culturele verschillen en emoties van invloed zijn op de mening van studenten over toetsing en hun leerstijl. Daarnaast bleek dat een combinatie van formatieve en summatieve toetsing op basis van leerdoelen een manier is om te bewerkstelligen dat toetsing een effect heeft op het onderwijs. Bij het samenstellen van deze combinatie dient rekening gehouden te worden met de culturele achtergrond van studenten, waarden en het toegepaste onderwijssysteem. Als deze factoren in evenwicht zijn, kan dit studenten motiveren om wenselijke leerstrategieën te blijven toepassen en de mogelijke negatieve effecten van het verborgen curriculum beperken.

Hoofdstuk 5 beschrijft het vierde onderzoek met als doel het verwerven van inzicht in de invloed van de mening van studenten over zelfbeoordeling en de toepassing daarvan in de praktijk op de leerstrategieën van studenten in een "community of practice" in de kliniek. Het betrof een fenomenologisch, kwalitatief onderzoek met behulp van interviews met drie verschillende jaargroepen die verschilden met betrekking tot omgevingsfactoren. De interviews waren gericht op de mening van de studenten over factoren met betrekking tot zelfbeoordeling en hoe de studenten deze factoren interpreteerden, met name de factoren "zelfbeoordeling in de praktijk" en "feedback van docenten". Onderzocht werd hoe deze omgevingsfactoren van invloed waren op de leerstrategieën van de studenten. Uit de analayse van de interviews kwamen drie hoofdthema's naar voren: het strategisch toepassen van zelfbeoordeling, zelfbeoordeling en de leerstrategieën van de studenten, en feedback en de leerstrategieën van de studenten. Uit de resultaten bleek dat het toepassen van zelfbeoordeling niet past binnen een summatieve context en dat dit culturele voorbereiding behoeft. Daarnaast bleek dat van de twee belangrijkste componenten van het zelfbeoordelingsproces feedback de doelmatigste strategie was om de leerstrategieën van de studenten te verbeteren. Positieve resultaten van zelfbeoordeling die in de literatuur beschreven worden, lijken grotendeels te kunnen worden toegeschreven aan feedback. 
Hoofdstuk 6 beschrijft het vijfde onderzoek, een literatuurstudie over de effecten van de onderwijsomgeving, met name de invloed van toetsing op de leerstrategieën van de studenten. Er is gezocht naar relevante literatuur in Pubmed, PsychINFO en Medline zonder beperkingen ten aanzien van publicatiesoort of publicatiedatum. Het belangrijkste thema dat naar voren kwam in de literatuur was the functie van toetsing, onderscheiden in summatieve en formatieve toetsing, en een algemeen effect van toetsing op de leerstrategieën van de studenten. In de onderzochte literatuur werd de complexiteit benadrukt van de relatie tussen de leeromgeving, de ideeën van studenten over toetsingseisen, en de leerstrategieën van de studenten. Op grond van theoretische overwegingen werd gesteld dat veel extrinsieke en intrinsieke factoren een mediërende functie hebben ten aanzien van de leerstrategieëen die studenten hanteren in reactie op toetsing. Er bleek echter weinig onderzoek gepubliceerd te zijn over deze factoren. Op basis van de resultaten kan samenvattend geconcludeerd worden dat formatieve toetsing kan aanzetten tot diepgaand leren, terwijl summatieve toetsing vaak zal leiden tot een oppervlakkige leerstrategie. Deze effecten zijn echter niet duidelijk omschreven en verder onderzoek is nodig om meer inzicht te verwerven in de complexe relatie tussen toetsing en de manier waarop studenten leren.

Hoofdstuk 7 presenteert de algemene discussie van de onderzoeken in dit proefschrift. De onderzoeksresultaten worden besproken waarbij ook aandacht besteed wordt aan de beperkingen van de onderzoeken. Ook worden aanbevelingen gedaan op basis van de gevonden resultaten. Benadrukt wordt dat de meningen van studenten verschillen ten aanzien van toetsingsfactoren en dat dezelfde factoren niet altijd dezelfde studiestrategieën bevorderen. Zo leidt summatieve toetsing voor sommige studenten tot oppervlakking leren, maar kan het andere studenten aanzetten tot diepgaand leren; hetzelfde fenomeen doet zich voor bij formatieve toetsing. De verschillen tussen de gezichtspunten in de literatuur ten aanzien van summatieve en formatieve toetsing zijn mogelijk toe te schrijven aan de aanwezigheid van andere mediërende factoren die 
de leerstrategieën van studenten en hun mening over toetsing bepalen. Het is daarom noodzakelijk om te streven naar een evenwicht tussen de verschillende effecten van toetsingsfactoren op de leerstrategieën van studenten. Het zal echter moeilijk zijn om te komen tot een eenduidige, uniform toepasbare formule die het gewenste evenwicht tussen summatieve en formatieve toetsing kan bepalen voor alle omstandigheden, voor alle studenten, voor alle culturen en voor alle instellingen; verschillende situaties vragen elk om een verschillende, individuele aanpak. De inzichten uit de onderzoeken in dit proefschrift betreffende de invloed van toetsingsfactoren op de leerstrategieën van studenten hebben geleid tot de volgende aanbevelingen: 1) Het ontwerp van een curriculum moet aansluiten bij de betreffende onderwijscultuur, de faciliteiten, de carrièremogelijkheden en het gezondheidszorgsysteem. Dergelijk maatwerk zal bijdagen tot een successvolle invoering van het curriculum. 2) Inspanningen om de onderwijscultuur te verbeteren en de kloof tussen de meningen van studenten en docenten te overbruggen zullen studenten aanzetten tot toepassing van betere leerstrategieën gericht op diepgaand leren. 3) Afgezien van deze inzichten en aanbevelingen, hebben wij ook moeten vaststellen dat er in de literatuur een ernstig gebrek aan aandacht bestaat voor de relatie tussen toetsing en studiestrategieën. Verder onderzoek op dit gebied wordt dan ook sterk aanbevolen. 



\section{Curriculum Vitae}

Hanan M. Al-Kadri received her MD degree from the Medical School at Damascus University in 1986. Next, she obtained a joint Diploma in Obstetrics and Gynecology from the Ministry of Health at Kingdom of Saudi Arabia and the Royal College of Ireland in 1995. She completed her residency training at Riyadh Central Hospital and obtained both Arab and Saudi Board in Obstetrics and Gynecology, Ministry of Health, Saudi Arabia in 1997. Dr. Al-Kadri moved to work for the King Abdulaziz Medical City (KAMC), Riyadh in March 1999 and became a consultant in Obstetrics and Gynecology in 2002. In the department of Obstetrics and gynecology at KAMC, Riyadh, Dr. Al-Kadri worked as a Deputy Residency Training Program director between 2003 and 2005. She then worked as Residency Training Program director between 2005 and 2007. Later on with the establishment of King Saud bin Abduaziz University for Health Sciences, College of Medicine (KSAU-HS, COM) at KAMC, she moved to work as an Undergraduate Clinical Education Director for the Obstetrics and Gynecology medical block between 2007 and 2010 and was the Head Division for the General Obstetrics and Gynecology between 2007 and 2009. Subsequently she was appointed as the Associate Dean Female Medical Students at the KSAU-HS, COM.

Dr. Al-Kadri obtained Master in Health Profession Education (MHPE) from Maasticht University in 2008 with Cum Laude. During her studies, she developed interest in Students Assessment and started a PhD on Students Assessment under the supervision of Professor Cees van der Vleuten immediately after her MHPE completion.

Dr. Al-Kadri is an associate professor of Obstetrics and Gynecology and she serves on a number of medical and educational committees. She has interest in obstetrics patients' safety. She is principal or associate investigator on a number of grants, and has published over 25 peer-reviewed manuscripts. 



\section{SHE Dissertations Series}

Sok Ying Liaw, (14-12-2011) Rescuing A Patient In Deteriorating Situations (RAPIDS): A programmatic approach in developing and evaluating a simulation-based educational program.

Singaram, V.S. (7-12-2011) Exploring the Impact of Diversity Factors on ProblemBased Collaborative Learning.

Balslev, T. (24-11-2011) Learning to diagnose using patient video cases in paediatrics: Perceptive and cognitive processes.

Widyandana, D. (19-10-2011) Integrating Pre-clinical skills training in skills laboratory and primary health care centers to prepare medical students for their clerkships.

Durning, S.J. (09-09-2011) Exploring the Influence of Contextual Factors of the Clinical Encounter on Clinical Reasoning Success (Unraveling context specificity).

Govaerts, M.J.B. (08-09-2011) Climbing the Pyramid;Towards Understanding Performance Assessment.

Stalmeijer, R. E.(07-07-2011) Evaluating Clinical Teaching through Cognitive Apprenticeship.

Malling, B.V.G. (01-07-2011) Managing word-based postgraduate medical education in clinical departments.

Veldhuijzen, J.W. (17-06-2011) Challenging the patient-centred paradigm: designing feasible guidelines for doctor patient communication.

Van Blankenstein, F. (18-05-2011) Elaboration during problem-based, small group discussion: A new approach to study collaborative learning.

Van Mook, W. (13-05-2011) Teaching and assessment of professional behavior: Rhetoric and reality. 
De Leng, B. (8-12-2009). Wired for learning. How computers can support interaction in small group learning in higher education.

Maiorova, T. (29-05-2009). The role of gender in medical specialty choice and general practice preferences.

Bokken, L. (04-03-2009). Innovative use of simulated patients for educational purposes.

Wagenaar, A. (18-09-2008). Learning in internships. What and how students learn from experience.

Driessen, E. (25-06-2008). Educating the self-critical doctor. Using portfolio to stimulate and assess medical students' reflection.

Derkx, H. (18-06-2008). For your ears only. Quality of telephone triage at out-of-hours centres in the Netherlands.

Niessen, Th. (30-11-2007). Emerging epistemologies: making sense of teaching practice.

Budé, L. (05-10-2007). On the improvement of students' conceptual understanding in statistics education.

Niemantsverdriet, S. (26-07-2007). Learning from international internships: A reconstruction in the medical domain.

Marambe, K. (20-06-2007). Patterns of student learning in medical education - A Sri Lankan study in traditional curriculum.

Pleijers, A. (19-01-2007). Tutorial group discussion in problem-based learning.

Sargeant, J. (21-09-2006). Multi-source feedback for physician learning and change.

Dornan, T. (12-06-2006). Experience-based learning. 
Wass, V. (12-05-2006). The assessment of clinical competence in high stakes examinations.

Prince, K. (21-04-2006). Problem-based learning as a preparation for professional practice. 
